\title{
Fermion and photon gap-equations in Minkowski space within the Nakanishi integral representation method
}

\author{
Cédric Mezrag ${ }^{1,2, a}$, Giovanni Salmè ${ }^{1, b}$ \\ ${ }^{1}$ Istituto Nazionale di Fisica Nucleare, Sezione di Roma, P.le A. Moro 2, 00185 Rome, Italy \\ ${ }^{2}$ IRFU, CEA, Université Paris-Saclay, 91191 Gif-sur-Yvette, France
}

Received: 2 October 2020 / Accepted: 23 December 2020 / Published online: 16 January 2021

(C) The Author(s) 2021

\begin{abstract}
The approach based on the Nakanishi integral representation of $\mathrm{n}$-leg transition amplitudes is extended to the treatment of the self-energies of a fermion and an (IRregulated) vector boson, in order to pave the way for constructing a comprehensive application of the technique to both gap- and Bethe-Salpeter equations, in Minkowski space. The achieved result, namely a 6-channel coupled system of integral equations, eventually allows one to determine the three Källén-Lehman weights for fully dressing the propagators of fermion and photon. A first consistency check is also provided. The presented formal elaboration points to embed the characteristics of the non-perturbative regime at a more fundamental level. It yields a viable tool in Minkowski space for the phenomenological investigation of strongly interacting theories, within a QFT framework where the dynamical ingredients are made transparent and under control.
\end{abstract}

\section{Introduction}

The description of bound states, fully taking into account the general principles of the relativistic quantum field theory (QFT) and the needed non-perturbative regimes, is a longstanding and highly challenging problem. As it is wellknown, the formal solution of the problem can be traced back to the birth of relativistic QFT, with the seminal paper by Salpeter and Bethe [1]. Starting from the analysis of the pole contributions to the Green's function relevant for the bound state under scrutiny (e.g., the four-points Green's function for investigating two-body bound states), they introduced an integral equation, known as the Bethe-Salpeter equation (BSE) for the bound-state amplitude, where the kernel is obtained from the two-particle irreducible diagrams, describing the dynamics inside the system. The systematic evalua-

\footnotetext{
a e-mail: cedric.mezrag@cea.fr

b e-mail: salmeg@roma1.infn.it (corresponding author)
}

tion of the interaction kernel needs in turn the knowledge of other key ingredients: (i) self-energies of both intermediate particles and quanta and (ii) vertex functions, as pointed out by Gell-Mann and Low [2]. Unfortunately, those 2- and 3point functions are quantities to be determined through the infinite tower of Dyson-Schwinger equations (DSEs) [3-5] (see for introductory reviews, e.g., Refs. [6-11], and references quoted therein) that govern the whole set of $N$-point functions. Therefore, in order to make feasible the construction of more and more realistic interaction kernels, model builders have to elaborate strategies for truncating the DSEs infinite tower, as much self-consistently as possible, while retaining the dynamical effects, at the greatest extent (see, e.g., Ref. [12] for a closed-form of the BSE kernel, obtained by using the acting symmetries). Finally, it is worth mentioning that within the Hamiltonian framework (suitable for the studies in Minkowski space), other relevant non-perturbative approaches, have been developed, like (i) the discretized light-cone quantization [13], e.g. recently applied to positronium, pion and kaon by using the so-called Basis Light-front Quantization and a suitable truncation of the Fock space (see, e.g., Refs. [14-16]); and (ii) the Hamiltonian formulation of the lattice gauge theories that preserves the evolution of the states with a continuous real time [17], (see, e.g., Ref. [18] and references quoted therein for a recent QCD study and Ref. [19] for interesting quantum simulations of lattice gauge theories).

In the last decades, significant progresses have been done for implementing the approach based on BSE plus truncated DSEs, ${ }^{1}$ and a high degree of sophistication has been achieved, mainly in Euclidean space (see Refs. [6-11,20] and also Refs. [21-27]). Moreover, as it is well-known, DSEs can be obtained in a very efficient way by using the path-

\footnotetext{
1 We can roughly group the truncation schemes in two sets: (i) the ones exploiting a dressed vertex, with different amount of complexity, and (ii) the ones using a simple bare vertex.
} 
integral formalism, that in turn acquires a rigorous mathematical meaning in the Euclidean space (see e.g. Ref. $[28,29]$ ). ${ }^{2}$ In particular, the most widely investigated field is the continuum QCD, with an impressive wealth of applications in hadron physics, ranging from baryon and meson spectra to elastic electromagnetic $(\mathrm{em})$ form factors and transition ones (see, e.g., [6-11,20]). Notice that also some timelike observables can be studied, e.g. by exploiting (i) analytic models for the running coupling (see the seminal Ref. [31]); (ii) the evaluation of a finite number of moments of proper Euclidean correlation functions (see, e.g., Ref. [32] for a recent approach on lattice QCD and Refs. [33,34] for continuum QCD results); (iii) integral representations of the quantities primarily evaluated in Euclidean space or algebraic Ansätze, properly tuned through spacelike data (see, e.g., [35-39]).

Furthermore, also applications to QED have been pursued at large extent (see, e.g., Refs. [40-43] and for a recent study in Minkowski space Ref. [44]). The interest in investigating the QED, in the whole dynamical range, may be surprising, given the extraordinary accuracy achieved in the comparison with the data by using perturbative tools (see, e.g., the case of the muon anomalous magnetic moment [45]). Indeed, a close study of the non-perturbative regime on the one side is relevant for shedding light on QED at very short distances, as suggested, e.g., in Refs. [46,47] for Euclidean studies of the critical coupling, below which chiral symmetry breaks down in quenched QED, and in Ref. [48] for an Euclidean investigation on how to escape the triviality fate of QED by adding a relevant four-fermion operator. On the other side, a nonperturbative exploration of QED represents a needed step for approaches played in Minkowski space, and eventually aiming to compare the calculated outcomes with experimental results for hadrons, as it has been already done by using approaches in Euclidean space.

Our investigation will focus on the study of the charged fermion and photon gap-equations below the critical coupling, $\alpha_{c} \leq \pi / 3$, (see, e.g., Refs. [46,47,49]), and in order to help the reader to better appreciate the differences with other approaches it is useful to indicate, even in a simplistic way, the directions along which we will move in what follows. For this reason, let us immediately mention the two key ingredients we will adopt: (i) the Minkowski space, where the physical processes take place and (ii) the structure of the vertex function.

The vertex has a prominent role, and in our approach it is composed of two contributions. The first term is the wellknown part introduced by Ball and Chiu in Ref. [50], that fulfills the Ward-Takahashi identities (WTIs) (both the differential form and the finite-difference one). The second term

\footnotetext{
2 It must recalled that the complete equivalence between Euclidean and relativistic QFT, under a given set of necessary and sufficient conditions, was established by Osterwalder and Schrader, in the seventies [30].
}

is a transverse contribution (see, e.g., Refs. [40,42,44,51-54] for a wide discussion), based on a minimal Ansätz proposed in Ref. [22]. Such a transverse term is able to restore the full multiplicative renormalization of both fermion and photon propagators (solutions of suitably truncated DSEs), and in turn gracefully implements a workable, self-consistent truncation scheme. Indeed, although the knowledge of the full content of the vertex requires the one of the full off-shell scattering matrix, the longitudinal WTI and its transverse counterparts relate respectively the divergence and the curl of the vertex in terms of the fermion 2-points function. These relations are exact for the divergence and truncated for the curl, allowing for a viable closed system [12,22].

Another important ingredient, though more technical, is represented by the so-called Nakanishi integral representation (NIR) of a generic $n$-leg transition amplitude [55-57]. Indeed, such a tool has allowed one to undertake new efforts for developing methods for solving in Minkowski space both truncated DSEs (see, e.g., Refs. [44,58-63]) and BSE (see, e.g., Refs. [64-78], where systems with and without spin degrees of freedom are investigated).

The main motivation for adopting the NIR, closely related to the Stieltjes transform (see an application in Ref. [79]), is given by the possibility to express the $n$-leg transition amplitudes through their all-order perturbative form. The freedom needed for exploring a non-perturbative regime is assured via the unknown Nakanishi weight functions (NWFs), that are real functions fulfilling a uniqueness theorem, within the Feynman diagrammatic framework [57]. Such a freedom has shown all its relevance in the numerical studies of the bound states (by using both ladder and cross-ladder interaction kernels), that are the main instance where the realistic description of the non-perturbative regime is necessary. Furthermore, the NWFs to be used for the self-energies do not depend upon the external momenta, greatly simplifying our formal elaboration, as shown in what follows. The advantage of the NIR is that the four-momentum dependence is made explicit, allowing direct algebraic manipulations, and eventually making affordable analytic integrations. This is an important virtue of the NIR approach, since it simplifies the treatment of the expected singularities. On the phenomenology side, when light-cone observables have to be evaluated, e.g. for describing the partonic structure of hadrons $[35,80$ 88], the explicit dependence upon the momenta facilitates the needed projection onto the light-cone. However, in the NIR context, the dynamical assumptions are still much simpler than the one made in Euclidean calculations. For instance, in the above mentioned works when solving Minkowskian BSE for mesons, the constituent fermions are most of the time considered perturbative-like, i.e. omitting the running of the dressed quark mass (with the exception of Ref. [76] where it has been proposed to import the running mass of the quarks from the Euclidean lattice into the BSE framework). 
Our present effort aims at formally developing a method based on NIR for solving a coupled system composed by the gap-equations for both fermion and gauge boson, directly in Minkowski space. It should be pointed out that the final goal (to be presented elsewhere) of the program we are pursuing is to provide both implementation and quantitative solutions of the BSE with dressed propagators, in order to achieve a more and more realistic description of an interacting system within the QFT framework, directly in the physical space. The integral-equation system, we arrive at, is obtained by using a self-consistent truncation scheme of DSEs, valid in the whole dynamical range of QED, and by adopting both dimensional regularization and momentum-subtraction procedure for the renormalization. Moreover, to pragmatically remove the well-known IR divergences, a tiny mass-regulator has been introduced for the gauge boson, (see, e.g., Ref. [89] for a more general discussion and Ref. [90] for a recent analysis). In general, we share the same spirit of works as: (i) Ref. [44] where, within a quenched approximation, a spectral representation of the fermion propagator was adopted, in combination with its Källén-Lehman (KL) representation, and, importantly, a vertex function was constructed by exploiting the form suggested by the Gauge Technique [91] plus transverse terms, added for matching the perturbative expressions of the renormalized fermion self-energy (see also Refs. [77,92] for further Minkowskian exploration of a massive QED, in quenched approximation); (ii) Refs. [58,60,61] (see also Ref. [93] for a first study of the transverse vertex contribution), where a more direct link to the NIR technique (with different sets of approximations) can be found. Simplifying, the main difference with the previous works is a fully dressing of fermion and photon self-energies, by introducing a vertex function composed by the standard Ball-Chiu component [50] and a minimal Ansätz for the purely transverse contribution [22], able to ensure the multiplicative renormalizability of the whole approach.

The paper is organized as follows. In Sect. 2, the general formalism is introduced for fermion and photon propagators and self-energies, in terms of the KL representations and the NIR, respectively. In Sect. 3, the adopted vertex function is discussed. In Sect. 4, the gap-equations are introduced and the main result of our formal analysis, i.e. the coupled system of integral equations for determining the NWFs, of both electron and photon self-energies, is illustrated. In Sect. 5 an initial application of the coupled system, based on its first iteration, is shown. In Sect. 6, the conclusions of our analysis of the truncated DSEs within the NIR framework are drawn and the perspectives of the future numerical studies are presented. Finally, it has to be emphasized that the Appendices have been written in a detailed form for making as simple as possible a check of the whole formalism, and therefore they have to be considered an essential part of the work.

\section{General formalism}

In this section, we summarize the general formalism that will be used in our investigation of QED in Minkowski space (see the review in Ref. [6] for the Euclidean version). We introduce first the expression of the self-energy (2-leg transition amplitude in the Nakanishi language [57], that emphasizes the set of external momenta) in terms of NIR, for both fermion and photon. Then, the KL representations of the corresponding propagators are given. The main goal of this initial step is the relations between KL weights and NWFs (see Refs. $[60,61]$, for an analogous approach, but with renormalization constants $Z_{1}=Z_{2}=1$ and with a bare vertex function or the Ball-Chiu one, respectively).

The suitable renormalization scheme we adopt is the momentum subtraction one, applied on the mass-shell (MOM), as discussed in what follows. This scheme is suitable when asymptotic states exist, and this property is actually needed for the KL representation we have adopted. Clearly, when a confined phase of the QED establishes, likely for large values of photon running mass, the KL representation becomes unproved (see, e.g., Ref. [47] and references quoted therein for the analysis of the confining phase in QED within the gap-equation formalism in Euclidean space, and Ref. [94] for a more recent investigation in Minkowski space). It should be anticipated that both electron and photon self-energies can be nicely renormalized by applying such a scheme, given the benefit from the presence of the transverse component of the vertex function.

\subsection{The renormalized propagator of a fermion}

By adapting the notations in Ref. [6], one can write the following relations involving the renormalized propagator of a fermion and the regularized self-energy.

The renormalized fermion propagator is given by

$S_{R}(\zeta, p)=\frac{i}{p-m(\zeta)-\Sigma_{R}(\zeta ; p)+i \epsilon}$

with $\zeta$ the renormalization point and $\Sigma_{R}(\zeta ; p)$ the renormalized self-energy. From Lorentz invariance, one can write

$\Sigma_{R}(\zeta ; p)=p \mathcal{A}_{R}(\zeta ; p)+\mathcal{B}_{R}(\zeta ; p)$,

with $\mathcal{A}_{R}(\zeta ; p)$ and $\mathcal{B}_{R}(\zeta ; p)$ suitable scalar functions. In terms of the expression in Eq. (2), the renormalized propagator reads

$$
\begin{aligned}
& S_{R}(\zeta, p) \\
& =i \frac{p\left(1-\mathcal{A}_{R}(\zeta ; p)\right)+m(\zeta)+\mathcal{B}_{R}(\zeta ; p)}{p^{2}\left(1-\mathcal{A}_{R}(\zeta ; p)\right)^{2}-\left(m(\zeta)+\mathcal{B}_{R}(\zeta ; p)\right)^{2}+i \epsilon}
\end{aligned}
$$


Noteworthy, by requesting that the renormalized propagator for $p^{2} \rightarrow \zeta^{2}$ has a pole at the mass $m(\zeta)=m_{\text {phys }}{ }^{3}$ and the same residue of the free propagator, one finds the constraints to be fulfilled by the two scalar functions, at the renormalization point. Needless to say, those constraints are crucial for establishing the relations between the regularized self-energy and the two renormalization constants $\delta m$ and $Z_{2}(\zeta, \Lambda$ ) (see what follows). As a matter of fact, from the well-known general approach illustrated, e.g., in Ref. [89] (or adopting Eq. (3) and imposing $-i\left(p^{2}-m^{2}(\zeta)\right) S_{R}(\zeta ; p) \rightarrow p_{\text {on }}+m(\zeta)$ for $p \rightarrow p_{o n}$, with $\left.p_{o n}^{2}=m^{2}(\zeta)\right)$ one gets

$$
\begin{aligned}
& m(\zeta) \mathcal{A}_{R}(\zeta ; \zeta)+\mathcal{B}_{R}(\zeta ; \zeta)=0 \\
& \mathcal{A}_{R}(\zeta ; \zeta)+2 m(\zeta) \\
& \quad \times\left[m(\zeta) \frac{\partial \mathcal{A}_{R}(\zeta ; p)}{\partial p^{2}}+\frac{\partial \mathcal{B}_{R}(\zeta ; p)}{\partial p^{2}}\right]_{p^{2}=\zeta^{2}}=0
\end{aligned}
$$

These two equations define the standard on-shell QED renormalization scheme. Bringing in mind that the natural outcome of our formal elaboration will be a system of integral equations, needed for determining $\mathcal{A}_{R}$ and $\mathcal{B}_{R}$, we adopt the following renormalization conditions defining the RI'/MOM scheme (see Ref. [95], for the renormalization independent method in the unquenched QED)

$\mathcal{A}_{R}(\zeta ; \zeta)=0, \quad \mathcal{B}_{R}(\zeta ; \zeta)=0$.

It is worth noticing the following remarks about this choice: (i) it preserves the pole at the physical mass of the fermion; (ii) it allows a numerical simplification, avoiding to implement boundary conditions where there is an interplay between $\mathcal{A}_{R}$ and $\mathcal{B}_{R}$; and last but not least (iii) exchanging the physical mass for the current mass evaluated at a space-like momentum, it is formally similar to the RI'/MOM scheme exploited in the literature devoted to the non-perturbative studies of QFT, e.g. in the context of the investigation on the lattice (see the discussions on the RI'/MOM scheme e.g., in Refs. $[96,97]$ ) as well as in continuous approaches (see, e.g., Refs. $[6,95])$. Since at the present stage of the novel approach we are exploring, the two boundary conditions in Eq. (5) turn out to simplify the determination of the two renormalization constants, we will leave the study of QED in the standard renormalization scheme, Eq. (4), for further investigation.

The propagator $S_{R}$ can be expressed in terms of the regularized quantity, $\Sigma(\zeta, \Lambda ; p)$, where $\Lambda$ stands for a Poincaré invariant regulator, e.g. $\Lambda=1 / \epsilon$ within a dimensional regularization framework with $d=4-\epsilon$. To make the mathematical notation less heavy, in what follows it is understood that the relations involving renormalized quantities hold only in the limit $\Lambda \rightarrow \infty$ (notice that above the critical coupling,

\footnotetext{
${ }^{3}$ For the sake of generality, we will leave the notation $m(\zeta)$ in the following expressions, though an on-mass-shell renormalization is adopted.
}

one expects to meet well-known difficulties for QED, as illustrated e.g., in Refs. $[48,95])$. Hence, one writes

$$
\begin{aligned}
& S_{R}(\zeta, p)=\frac{1}{Z_{2}(\zeta, \Lambda)} S(\zeta, \Lambda ; k) \\
& \quad=\frac{1}{Z_{2}(\zeta, \Lambda)} \frac{i}{p-m(\zeta)+\delta m-\Sigma(\zeta, \Lambda ; p)+i \epsilon},
\end{aligned}
$$

where $Z_{2}(\zeta, \Lambda)$ is the renormalization factor affecting the fermionic field and $\delta m=m(\zeta)-m_{0}$, with $m_{0}$ the bare mass. The analogous form of Eq. (2), for the regularized self-energy reads (it is useful to include the renormalization constant $Z_{2}$ in the definition)

$$
\begin{aligned}
& \Sigma_{Z}(\zeta, \Lambda ; p)=Z_{2}(\zeta, \Lambda) \Sigma(\zeta, \Lambda ; p) \\
& \quad=p \mathcal{A}_{Z}(\zeta, \Lambda ; p)+\mathcal{B}_{Z}(\zeta, \Lambda ; p)
\end{aligned}
$$

with $\mathcal{A}_{Z}(\zeta, \Lambda ; p)$ and $\mathcal{B}_{Z}(\zeta, \Lambda ; p)$ suitable scalar functions. In particular, comparing Eq. (1) and Eq. (6), one obtains

$$
\begin{aligned}
\mathcal{A}_{R}(\zeta ; p)= & \mathcal{A}_{Z}(\zeta, \Lambda ; p)-\left(Z_{2}(\zeta, \Lambda)-1\right), \\
\mathcal{B}_{R}(\zeta ; p)= & \mathcal{B}_{Z}(\zeta, \Lambda ; p)-\left[m(\zeta)\left(1-Z_{2}(\zeta, \Lambda)\right)\right. \\
& \left.+Z_{2}(\zeta, \Lambda) \delta m\right]
\end{aligned}
$$

Indeed, those relations amount to the outcomes of the subtraction scheme for the renormalization of each scalar function. Moreover, by taking into account Eq. (5), one has

$$
\begin{aligned}
& \mathcal{A}_{Z}(\zeta, \Lambda ; \zeta)=Z_{2}(\zeta, \Lambda)-1, \\
& \mathcal{B}_{Z}(\zeta, \Lambda ; \zeta)=m(\zeta)\left(1-Z_{2}(\zeta, \Lambda)\right)+Z_{2}(\zeta, \Lambda) \delta m,
\end{aligned}
$$

and therefore in the limit $\Lambda \rightarrow \infty$ :

$$
\begin{aligned}
\Sigma_{R}(\zeta ; p)= & \Sigma_{Z}(\zeta, \Lambda ; p)-\left.\Sigma_{Z}(\zeta, \Lambda ; p)\right|_{p^{2}=\zeta^{2}} \\
= & p\left[\mathcal{A}_{Z}(\zeta, \Lambda ; p)-\left.\mathcal{A}_{Z}(\zeta, \Lambda ; p)\right|_{p^{2}=\zeta^{2}}\right] \\
& +\left[\mathcal{B}_{Z}(\zeta, \Lambda ; p)-\left.\mathcal{B}_{Z}(\zeta, \Lambda ; p)\right|_{p^{2}=\zeta^{2}}\right]
\end{aligned}
$$

Pursuing our goal of establishing a formal framework where one can get actual solutions of the gap equation, and eventually describe the renormalized propagator, we usefully introduce the NIR for the fermionic self-energy. This can be achieved by starting from the approach proposed for a scalar case by Nakanishi (see Ref. [57]), for summing up the infinite contributions to a given n-leg amplitude, and generalizing in two respects. One is the transition from scalars to fermions, and the second one, more important, from a perturbative to a non-perturbative regime. Those steps have been explored for the BSEs in Refs. [64-78]. For the fermion self-energy, it is necessary to introduce two NWFs, since one has to deal with two scalar functions. Hence, the regularized self-energy can be written in terms of the following scalar functions

$$
\mathcal{A}_{Z}(\zeta, \Lambda ; p)=\int_{s_{t h}}^{\infty} d s \frac{\rho_{A}(s, \zeta, \Lambda)}{p^{2}-s+i \epsilon},
$$


$\mathcal{B}_{Z}(\zeta, \Lambda ; p)=\int_{s_{t h}}^{\infty} d s \frac{\rho_{B}(s, \zeta, \Lambda)}{p^{2}-s+i \epsilon}$

with $s_{t h}$ the multiparticle threshold and $\rho_{A(B)}$ the NWFs. It should be recalled that the NWFs are real functions, and do not depend upon the external momenta. This last remark will be useful for simplifying the formal elaboration aiming to get the suitable integral equations for $\rho_{A(B)}$.

Moreover, the NWFs have to fulfill the relation entailed by Eq. (9), i.e.

$$
\begin{aligned}
& Z_{2}(\zeta, \Lambda)=1+\int_{s_{t h}}^{\infty} d s \frac{\rho_{A}(s, \zeta, \Lambda)}{\zeta^{2}-s+i \epsilon} \\
& Z_{2}(\zeta, \Lambda) \delta m=\int_{s_{t h}}^{\infty} d s \frac{m(\zeta) \rho_{A}(s, \zeta, \Lambda)+\rho_{B}(s, \zeta, \Lambda)}{\zeta^{2}-s+i \epsilon}
\end{aligned}
$$

It is easily seen that NWFs with a constant behavior for $s \rightarrow$ $\infty$ generate an expected logarithmic divergence.

By using Eqs. (8), (9) and (11) one can write

$$
\begin{aligned}
\mathcal{A}_{R}(\zeta ; p) & =\lim _{\Lambda \rightarrow \infty}\left[\mathcal{A}_{Z}(\zeta, \Lambda ; p)-\left.\mathcal{A}_{Z}(\zeta, \Lambda ; p)\right|_{p^{2}=\zeta^{2}}\right] \\
& =\left(\zeta^{2}-p^{2}\right) \int_{s_{t h}}^{\infty} d s \frac{\rho_{A}(s, \zeta)}{\left(p^{2}-s+i \epsilon\right)\left(\zeta^{2}-s+i \epsilon\right)}, \\
\mathcal{B}_{R}(\zeta ; p) & =\lim _{\Lambda \rightarrow \infty}\left[\mathcal{B}_{Z}(\zeta, \Lambda ; p)-\left.\mathcal{B}_{Z}(\zeta, \Lambda ; p)\right|_{p^{2}=\zeta^{2}}\right] \\
& =\left(\zeta^{2}-p^{2}\right) \int_{s_{t h}}^{\infty} d s \frac{\rho_{B}(s, \zeta)}{\left(p^{2}-s+i \epsilon\right)\left(\zeta^{2}-s+i \epsilon\right)},
\end{aligned}
$$

where the notation $\rho_{A(B)}(s, \zeta)=\rho_{A(B)}(s, \zeta, \Lambda \rightarrow \infty)$ is adopted from now on.

It should be pointed out that the actual form of the NFWs will follow from the solution of the coupled system of integral equations we are going to elaborate. Anticipating on the next sections, a possible constant behavior of the NWFs $\rho_{A(B)}$ for $s \rightarrow \infty$ would be regularized by the quadratic dependence upon $s$ in the denominator, allowing to safely take $\Lambda \rightarrow \infty$. A situation in which $\rho_{A(B)}$ is not bounded at infinity would create regulator dependent results, and may appear above the critical coupling in QED as already mentioned.

Dealing with the gap-equations, it is fruitful to use the KL representation of the renormalized propagators, and therefore one has to establish the relation between KL weights and NWFs of the corresponding self-energy (see also Ref. [58] for the scalar case and Refs. [60,61] for $Q E D_{3+1}$ ). Recalling the following KL representation

$$
\begin{aligned}
S_{R}(\zeta, p)= & i \mathcal{R}_{S} \frac{p+m(\zeta)}{p^{2}-m^{2}(\zeta)+i \epsilon} \\
& +i \int_{s_{t h}}^{\infty} d s \frac{\not p \sigma_{V}(s, \zeta)+\sigma_{S}(s, \zeta)}{p^{2}-s+i \epsilon},
\end{aligned}
$$

where $\mathcal{R}_{S}$ is the fermion propagator residue, controlled by the choice of the renormalization scheme (here we have adopted RI'/MOM). Using $\Sigma_{R}(\zeta, p)$ from Eq. (2), one gets

$$
\begin{aligned}
i \int_{s_{t h}}^{\infty} & d s \frac{\not p \sigma_{V}(s, \zeta)+\sigma_{S}(s, \zeta)}{p^{2}-s+i \epsilon} \\
\quad= & i \frac{p\left(1-\mathcal{A}_{R}(\zeta ; p)\right)+m(\zeta)+\mathcal{B}_{R}(\zeta ; p)}{\mathcal{D}(p ; \zeta)+i \epsilon} \\
& \quad-i \mathcal{R}_{S} \frac{p+m(\zeta)}{p^{2}-m^{2}(\zeta)+i \epsilon},
\end{aligned}
$$

with

$\mathcal{D}(p ; \zeta)=p^{2}\left(1-\mathcal{A}_{R}(\zeta ; p)\right)^{2}-\left(m(\zeta)+\mathcal{B}_{R}(\zeta ; p)\right)^{2}$

By evaluating the needed traces, one can obtain the following relations

$$
\begin{aligned}
\int_{s_{t h}}^{\infty} d s \frac{\sigma_{V}(s, \zeta)}{p^{2}-s+i \epsilon}= & \frac{1-\mathcal{A}_{R}(\zeta ; p)}{\mathcal{D}(p ; \zeta)+i \epsilon}-\frac{\mathcal{R}_{S}}{p^{2}-m^{2}(\zeta)+i \epsilon} \\
\int_{s_{t h}}^{\infty} d s \frac{\sigma_{S}(s, \zeta)}{p^{2}-s+i \epsilon}= & \frac{m(\zeta)+\mathcal{B}_{R}(\zeta ; p)}{\mathcal{D}(p ; \zeta)+i \epsilon} \\
& -\frac{\mathcal{R}_{S} m(\zeta)}{p^{2}-m^{2}(\zeta)+i \epsilon}
\end{aligned}
$$

If one assumes that both KL weights and NWFs match the hypotheses for applying the Sokhotski-Plemelj formula, that reads

$\int_{-\infty}^{\infty} d s \frac{f(s)}{\omega-s+i \epsilon}=\mathrm{PV}\left[\frac{f(s)}{\omega-s}\right]-i \pi f(\omega)$,

with an understood $\theta\left(s-s_{t h}\right)$ inside $f(s)$, then one can manipulate the singular integrals in the lhs of Eq. (18) and the rhs of Eqs. (13) and (14) as follows

$$
\begin{aligned}
\int_{s_{t h}}^{\infty} d s & \frac{\sigma_{V(S)}\left(s^{\prime}, \zeta\right)}{\left(\omega-s^{\prime}+i \epsilon\right)} \\
= & \mathrm{PV}\left[\frac{\sigma_{V(S)}\left(s^{\prime}, \zeta\right)}{\left(\omega-s^{\prime}\right)}\right]-i \pi \sigma_{V(S)}(\omega, \zeta), \\
\int_{s_{t h}}^{\infty} d s & \frac{\rho_{A(B)}(s, \zeta)}{(\omega-s+i \epsilon)\left(\zeta^{2}-s+i \epsilon\right)} \\
= & \mathrm{PV}\left[\frac{\rho_{A(B)}(s, \zeta)}{(\omega-s)\left(\zeta^{2}-s\right)}\right]-i \pi \frac{\rho_{A(B)}(\omega, \zeta)}{\left(\zeta^{2}-\omega\right)} .
\end{aligned}
$$

Let us recall that $\rho_{A(B)}\left(s=\zeta^{2}, \zeta\right)=0$ and values $\omega \geq s_{t h}$ are relevant in what follows. By inserting Eqs. (21) in (13) and (14), the real and the imaginary parts of $\mathcal{A}_{R}(\zeta ; \omega)$ become

$$
\begin{aligned}
& \Re e\left\{\mathcal{A}_{R}(\zeta ; \omega)\right\}=\left(\zeta^{2}-\omega\right)\left\langle\rho_{A}\right\rangle, \\
& \Im m\left\{\mathcal{A}_{R}(\zeta ; \omega)\right\}=-\pi \rho_{A}(\omega, \zeta),
\end{aligned}
$$

with the notation $\left\langle\rho_{A}\right\rangle$ indicating the principal value in Eq. (21). Analogous expressions hold for $\mathcal{B}_{R}(\zeta ; \omega)$. Hence, one 
can formally gets the following relations between KL weights and NWFs for $\omega>\omega_{t h}=s_{t h}$

$\begin{aligned} \sigma_{V}(\omega, \zeta) & =\frac{D_{I}\left[1-\left(\zeta^{2}-\omega\right)\left\langle\rho_{A}\right\rangle\right]-\rho_{A}(\omega, \zeta) D_{R}}{D_{R}^{2}+\pi^{2} D_{I}^{2}} \\ \sigma_{S}(\omega, \zeta) & =\frac{D_{I}\left[m(\zeta)+\left(\zeta^{2}-\omega\right)\left\langle\rho_{B}\right\rangle\right]+\rho_{B}(\omega, \zeta) D_{R}}{D_{R}^{2}+\pi^{2} D_{I}^{2}},\end{aligned}$

where

$$
\begin{aligned}
D_{R}= & \omega\left[\left(1-\left(\zeta^{2}-\omega\right)\left\langle\rho_{A}\right\rangle\right)^{2}-\pi^{2} \rho_{A}^{2}(\omega, \zeta)\right] \\
& -\left[\left(m(\zeta)+\left(\zeta^{2}-\omega\right)\left\langle\rho_{B}\right\rangle\right)^{2}-\pi^{2} \rho_{B}^{2}(\omega, \zeta)\right], \\
D_{I}= & 2 \omega \rho_{A}(\omega, \zeta)\left[1-\left(\zeta^{2}-\omega\right)\left\langle\rho_{A}\right\rangle\right] \\
& +2 \rho_{B}(\omega, \zeta)\left[m(\zeta)+\left(\zeta^{2}-\omega\right)\left\langle\rho_{B}\right\rangle\right] .
\end{aligned}
$$

It has to be pointed out that the knowledge of the KL weights $\sigma_{S(V)}(\omega, \zeta)$ for $\omega>\omega_{t h}$ is enough for determining the fermion propagator for all the possible values of $p^{2}$.

\subsection{The renormalized propagator of a photon}

In the Landau gauge, the free propagator of the photon reads

$D^{\mu \nu}(q)=-i \frac{T^{\mu \nu}(q)}{q^{2}-\zeta_{p}^{2}+i \epsilon}$,

where $T^{\mu \nu}(q)$ is the standard transverse projector

$T^{\mu \nu}(q)=g^{\mu \nu}-\frac{q^{\mu} q^{v}}{q^{2}}$,

with its useful properties,

$$
\begin{aligned}
T_{\mu \nu}(q) & =g_{\mu \nu}-\frac{q_{\mu} q_{v}}{q^{2}}, \quad T_{\mu \alpha}(q) T_{\nu}^{\alpha}(q)=T_{\mu \nu}(q) \\
T_{\mu \nu}(q) g^{v \mu} & =T_{\mu}^{\mu}(q)=3
\end{aligned}
$$

and $\zeta_{p}$ is a IR-regulator, (see, e.g., Ref. [89]). For the sake of light notation, the dependence upon $\zeta_{p}$ will be understood in the renormalized quantities. Hence, the renormalized photon propagator reads

$D_{R}^{\mu \nu}(\zeta, q)=-i \frac{T^{\mu \nu}}{\left(q^{2}-\zeta_{p}^{2}+i \epsilon\right)\left[1+\Pi_{R}(\zeta ; q)\right]}$,

where $\Pi_{R}(\zeta ; q)$ can be called the photon self-energy, fulfilling the following condition, able to lead to the correct residue at photon pole

$\Pi_{R}\left(\zeta ; \zeta_{p}\right)=0$

Notice that the photon propagator would present a problematic pole if there exists a critical value, $q_{\text {sing }}$, such that $1+\Pi_{R}\left(\zeta ; q_{\text {sing }}\right)=0$. Interestingly, the IR pole in (28) could be removed if also $\Pi_{R}\left(q^{2}\right)$ develops an IR pole, i.e. the so-called Schwinger mechanism [98], that leads to a massive photon (see, e.g., Ref. [99] for the absence of this phenomenon in $\mathrm{QED}_{3}$ and Ref. [100] for a comprehensive review in the case of QCD).

The relation between $D_{R}^{\mu \nu}(\zeta ; q)$ and both the regularized self-energy and the renormalization constant $Z_{3}(\zeta, \Lambda)$ is

$$
\begin{aligned}
& D_{R}^{\mu \nu}(\zeta ; q) \\
& \quad=-i \frac{T^{\mu \nu}}{Z_{3}(\zeta, \Lambda)\left(q^{2}-\zeta_{p}^{2}+i \epsilon\right)[1+\Pi(\zeta, \Lambda, q)]} .
\end{aligned}
$$

Comparing the denominators in Eqs. (28) and (30) one has for $\Lambda \rightarrow \infty$

$\Pi_{R}(\zeta, q)=\Pi_{Z}(\zeta, \Lambda ; q)+Z_{3}(\zeta, \Lambda)-1$,

with

$\Pi_{Z}(\zeta, \Lambda ; q)=Z_{3}(\zeta, \Lambda) \Pi(\zeta, \Lambda ; q)$

By imposing the condition in Eq. (29), one gets the following normalization

$\Pi_{Z}\left(\zeta, \Lambda ; \zeta_{p}\right)=1-Z_{3}(\zeta, \Lambda)$

and writes for $\Lambda \rightarrow \infty$

$\Pi_{R}(\zeta ; q)=\Pi_{Z}(\zeta, \Lambda ; q)-\Pi_{Z}\left(\zeta, \Lambda ; \zeta_{p}\right)$,

It is also useful to recall that the renormalized propagator fulfills the well-known integral equation, given by

$$
\begin{aligned}
D_{R}^{\mu \nu}(\zeta, q)= & D^{\mu v}(q)+D^{\mu \alpha}(q) \\
& \times\left[i \Pi_{\alpha \beta}^{R}(\zeta, q)\right] D_{R}^{\beta v}(\zeta, q),
\end{aligned}
$$

where $\Pi_{R}^{\mu \nu}\left(\zeta, q^{2}\right)$ is the renormalized vacuum polarization tensor, defined by

$\Pi_{R}^{\mu v}(\zeta, q)=-q^{2} T^{\mu v}(q) \Pi_{R}(\zeta ; q)$.

This quantity is involved in the gap-equation for the photon (see Sect. 4 for more details).

Analogously to the fermion case, one introduces the following NIR for $\Pi_{Z}(\zeta, \Lambda ; q)$

$\Pi_{Z}(\zeta, \Lambda ; q)=\int_{s_{t h}^{p}}^{\infty} d s \frac{\rho_{\gamma}(s, \zeta, \Lambda)}{\left(q^{2}-s+i \epsilon\right)}$,

where the real function $\rho_{\gamma}(s, \zeta, \Lambda)$ is the NWF for the regularized photon self-energy, and $s_{t h}^{p}$ the multiparticle threshold, i.e. $s_{t h}^{p}=4 m^{2}(\zeta)$.

Using Eqs. (32) and (36), one gets the following expression for $Z_{3}(\zeta, \Lambda)$

$Z_{3}(\zeta, \Lambda)=1-\int_{s_{t h}^{p}}^{\infty} d s \frac{\rho_{\gamma}(s, \zeta, \Lambda)}{\left(\zeta_{p}^{2}-s+i \epsilon\right)}$

The same observation below Eq. (12) is relevant also for Eq. (37). 
By exploiting Eqs. (32) and (36) in (33), $\Pi_{R}(\zeta ; q)$ can be written in terms of NWFs, viz

$$
\begin{aligned}
\Pi_{R}(\zeta ; q)= & \left(\zeta_{p}^{2}-q^{2}\right) \\
& \times \int_{s_{t h}^{p}}^{\infty} d s \frac{\rho_{\gamma}(s, \zeta)}{\left(\zeta_{p}^{2}-s+i \epsilon\right)\left(q^{2}-s+i \epsilon\right)},
\end{aligned}
$$

where $\rho_{\gamma}(s, \zeta)=\rho_{\gamma}(s, \zeta, \Lambda \rightarrow \infty)$.

The KL representation of $D_{\mu \nu}^{R}(\zeta, q)$ reads

$$
\begin{aligned}
D_{\mu \nu}^{R}(\zeta, q)= & -i T_{\mu \nu}(q) \\
& \times\left(\frac{1}{q^{2}-\zeta_{p}^{2}+i \epsilon}+\int_{s_{t h}^{p}}^{\infty} d s \frac{\sigma_{\gamma}(\omega, \zeta)}{q^{2}-s+i \epsilon}\right),
\end{aligned}
$$

and has to be compared with the following expression obtained from Eq. (28)

$$
\begin{aligned}
D_{R}^{\mu \nu}(\zeta, q)= & -i T_{\mu \nu}(q)\left[\frac{1}{q^{2}-\zeta_{p}^{2}+i \epsilon}\right. \\
& \left.-\frac{\Pi_{R}(\zeta ; q)}{\left(q^{2}-\zeta_{p}^{2}+i \epsilon\right)\left(1+\Pi_{R}(\zeta ; q)\right)}\right] .
\end{aligned}
$$

Hence one gets

$$
\int_{s_{t h}^{p}}^{\infty} d s \frac{\sigma_{\gamma}(s, \zeta)}{q^{2}-s+i \epsilon}=-\frac{\Pi_{R}(\zeta ; q)}{\left(q^{2}-\zeta_{p}^{2}+i \epsilon\right)\left(1+\Pi_{R}(\zeta ; q)\right)}
$$

By using Eqs. (19) and (38), the real and imaginary parts of $\Pi_{R}(\zeta ; q)$ can be easily written in terms of the NWF $\rho_{\gamma}(\omega, \zeta)$ as follows (recall that $q^{2} \geq s_{t h}^{p}$ )

$$
\begin{aligned}
& \Re e\left\{\Pi_{R}\left(\zeta ; q^{2}\right)\right\}=\left(q^{2}-\zeta_{p}^{2}\right) \mathrm{PV}\left[\frac{\rho_{\gamma}(s, \zeta)}{\left(q^{2}-s\right)\left(\zeta_{p}^{2}-s\right)}\right], \\
& \Im m\left\{\Pi_{R}\left(\zeta ; q^{2}\right)\right\}=-\pi \rho_{\gamma}\left(q^{2}, \zeta\right) .
\end{aligned}
$$

Finally, by using once more Eq. (19), one obtains the desired relation between $\rho_{\gamma}$ and $\sigma_{\gamma}$, given by

$$
\begin{aligned}
\sigma_{\gamma}(\omega, \zeta)= & -\frac{1}{\left(\omega-\zeta_{p}^{2}\right)} \\
& \times \frac{\rho_{\gamma}(\omega, \zeta)}{\left[\left(1+\left(\zeta_{p}^{2}-\omega\right)\left\langle\rho_{\gamma}\right\rangle\right)^{2}+\pi^{2} \rho_{\gamma}^{2}(\omega, \zeta)\right]},
\end{aligned}
$$

with $\omega \geq s_{\text {th }}^{p}$ and $\left\langle\rho_{\gamma}\right\rangle$ the principal value in Eq. (42).

\section{The renormalized vertex function}

The amputated three-leg transition amplitude, or vertex function, is the basic ingredient for any dynamical approach that aims at determining the self-energies of particle and quanta, involved in a given theory. Unfortunately, the fully dressed vertex function can be formally obtained only through the proper DSE where, in turn, the four-leg transition amplitude (i.e. the fully off-shell fermion-antifermion scattering kernel in the case of QED) is present. This fact makes clear the structure of the infinite tower of DSEs, where each n-leg transition amplitude fulfills an integral equation containing transition amplitudes with a number of legs greater than $n$. In spite of this, by using general principles, one can devise an overall form of the vertex, in terms of the Dirac structures allowed by both the Lorentz covariance, the parity conservation and time reversal (see, e.g., Refs [50,101]), when QED is investigated. Following well-known steps, one decomposes the vertex into two parts: (i) the standard component introduced in the early eighties by Ball and Chiu [50], in order to fulfill WTIs and to avoid any kinematical singularity, and (ii) a contribution purely transverse, i.e. containing the possible Dirac structures orthogonal to the momentum transfer $q=p_{f}-p_{i}$ (see Fig. 1. for the pictorial representation and the kinematics). As a matter of fact, one writes the renormalized vertex (or the regularized one, with the proper modification in the notations) as follows

$\Gamma_{R}^{\mu}\left(\zeta, p_{f}, p_{i}\right)=\Gamma_{R, B C}^{\mu}\left(\zeta, p_{f}, p_{i}\right)+\Gamma_{R, T}^{\mu}\left(\zeta, p_{f}, p_{i}\right)$

where $q \cdot \Gamma_{R, T}\left(\zeta, p_{f}, p_{i}\right)=0$ and $\Gamma_{R, B C}^{\mu}\left(\zeta, p_{f}, p_{i}\right)$ is the Ball-Chiu vertex dictated by the WTI i.e.

$$
\begin{aligned}
q \cdot & \Gamma_{R}\left(\zeta, p_{f}, p_{i}\right)=q \cdot \Gamma_{R, B C}\left(\zeta, p_{f}, p_{i}\right) \\
= & i S_{R}^{-1}\left(\zeta, p_{f}\right)-i S_{R}^{-1}\left(\zeta, p_{i}\right) \\
= & p_{f}-m(\zeta)-\Sigma_{R}\left(\zeta, p_{f}\right)-\left[p_{i}-m(\zeta)-\Sigma_{R}\left(\zeta, p_{i}\right)\right] \\
= & p_{f}\left[1-\mathcal{A}_{R}\left(\zeta ; p_{f}\right)\right]-\mathcal{B}_{R}\left(\zeta ; p_{f}\right) \\
& -p_{i}\left[1-\mathcal{A}_{R}\left(\zeta ; p_{i}\right)\right]+\mathcal{B}_{R}\left(\zeta ; p_{i}\right)
\end{aligned}
$$

The actual expression of $\Gamma_{R ; B C}^{\mu}[50]$, is given by

$$
\begin{aligned}
\Gamma_{R ; B C}^{\mu}\left(\zeta, p_{f}, k_{f}\right)= & \frac{\gamma^{\mu}}{2} F_{\mathcal{A}_{+}}\left(p_{f}, p_{i}, \zeta\right) \\
& -\frac{\left(p_{f}+p_{i}\right)\left(p_{f}+p_{i}\right)^{\mu}}{2} F_{\mathcal{A}_{-}}\left(p_{f}, p_{i}, \zeta\right) \\
& -\left(p_{f}+p_{i}\right)^{\mu} F_{\mathcal{B}}\left(p_{f}, p_{i}, \zeta\right)
\end{aligned}
$$

where

$$
\begin{aligned}
& F_{\mathcal{A}_{+}}\left(p_{f}, p_{i}, \zeta\right)=2-\mathcal{A}_{R}\left(\zeta ; p_{f}\right)-\mathcal{A}_{R}\left(\zeta ; p_{i}\right) \\
& =2+\int_{s_{t h}}^{\infty} d s \frac{\rho_{A}(s, \zeta)}{\left(\zeta^{2}-s+i \epsilon\right)} \\
& \quad \times\left[\frac{\left(p_{f}^{2}-\zeta^{2}\right)}{\left(p_{f}^{2}-s+i \epsilon\right)}+\frac{\left(p_{i}^{2}-\zeta^{2}\right)}{\left(p_{i}^{2}-s+i \epsilon\right)}\right] \\
& F_{\mathcal{A}_{-}}\left(p_{f}, p_{i}, \zeta\right)=\frac{\mathcal{A}_{R}\left(\zeta ; p_{f}\right)-\mathcal{A}_{R}\left(\zeta ; p_{i}\right)}{\left(p_{f}^{2}-p_{i}^{2}\right)}
\end{aligned}
$$




$$
\begin{gathered}
=-\int_{s_{t h}}^{\infty} d s \frac{\rho_{A}(s, \zeta)}{\left(p_{f}^{2}-s+i \epsilon\right)\left(p_{i}^{2}-s+i \epsilon\right)}, \\
F_{\mathcal{B}}\left(p_{f}, p_{i}, \zeta\right)=\frac{\mathcal{B}_{R}\left(\zeta ; p_{f}\right)-\mathcal{B}_{R}\left(\zeta ; p_{i}\right)}{\left(p_{f}^{2}-p_{i}^{2}\right)} \\
=-\int_{s_{t h}}^{\infty} d s \frac{\rho_{B}(s, \zeta)}{\left(p_{f}^{2}-s+i \epsilon\right)\left(p_{i}^{2}-s+i \epsilon\right)} .
\end{gathered}
$$

While $\Gamma_{R ; B C}^{\mu}$ is elaborated starting from WTIs and the crucial request of avoiding kinematical singularities, the transverse part $\Gamma_{R ; T}^{\mu}$ has to fulfill the constraint imposed by the curl of the current, $q^{\mu} \Gamma_{R}^{v}-q^{v} \Gamma_{R}^{\mu}$ [51] (see also the analysis in Ref. [22]), and it can be expressed in terms of eight Dirac structures, $T_{i}^{\mu}$, such that $q \cdot T_{i}=0$ (see Ref. [50] for the complete list) and eight scalar functions, $\mathcal{F}_{i}$, viz

$\Gamma_{R ; T}^{\mu}\left(\zeta, p_{f}, p_{i}\right)=\sum_{i=1,8} \mathcal{F}_{i}\left(p_{f}, p_{i}, \zeta\right) T_{i}^{\mu}\left(p_{f}, p_{i}\right)$

In general the functions $\mathcal{F}_{i}\left(p_{f}, p_{i}, \zeta\right)$ cannot be written only in terms of $\mathcal{A}_{R}$ and $\mathcal{B}_{R}$ [22], but the whole set of functions has to cooperate for ensuring another fundamental property: the multiplicative renormalizability of both self-energies (see Eqs. (6) and (30)) and vertex, viz

$$
\Gamma_{R}^{\mu}\left(\zeta, p_{f}, p_{i}\right)=Z_{1}(\zeta, \Lambda) \Gamma^{\mu}\left(\zeta, \Lambda ; p_{f}, p_{i}\right)
$$

with the constraint $Z_{1}(\zeta, \Lambda)=Z_{2}(\zeta, \Lambda)$. It is fundamental to notice that, given the DSEs, the multiplicative renormalizability of both two-leg and three-leg functions are intimately related. This has been elucidated by a vast literature, in different frameworks. In particular, in Refs. [40,42-44, 52, 54] (and references quoted therein) a close analysis, ranging from a first perturbative study to non-perturbative ones, was carried out, pointing to the role played by leading logarithms in determining the aforementioned property, through an unavoidable cooperation between the scalar functions present in $\Gamma_{R ; B C}^{\mu}$ and $\Gamma_{R ; T}^{\mu}$. Differently, in Refs. [40,53,102], within a quenched approximation, the requirement of multiplicative renormalization is implemented by looking for solutions of the fermion gap-equation with a power-law behavior.

In our unquenched approach, we take into account the transverse vertex, retaining only some contributions, as it will be explained in what follows. Indeed, this is a distinctive feature of our work, in comparison with approaches sharing the same spirit, i.e. exploiting spectral representations of both propagators and self-energies (see Refs. [44,58,60-63]). In particular we consider the following two Dirac structures, of the eight identified in Ref. [101],

$$
\begin{aligned}
T_{3}^{\mu}\left(p_{f}, p_{i}\right) & =q^{2} \gamma^{\mu}-q^{\mu} \phi \\
T_{8}^{\mu}\left(p_{f}, p_{i}\right) & =p_{f}^{\mu} p_{i}-p_{i}^{\mu} p_{f}-i \gamma^{\mu} \sigma_{\nu \rho} p_{i}^{v} p_{f}^{\rho} \\
& =-i \gamma_{5} \epsilon_{\alpha \nu \rho}^{\mu} \gamma^{\alpha} p_{i}^{v} q^{\rho}
\end{aligned}
$$

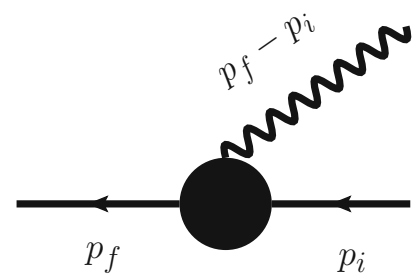

Fig. 1 The pictorial representation of the regularized fermion-photon vertex, with a fermion absorbing a photon

with $\sigma_{v \rho}=i\left[\gamma_{\nu}, \gamma_{\rho}\right] / 2$ and $\epsilon^{0123}=+1$. Notice that there is an overall different sign with respect to Ref. [50].

It is worth mentioning that in the fermion massless case (relevant for studying the dynamical generation of the mass) only the Dirac structures with $i=2,3,5,8$ contribute [42], and moreover, in the same limiting case, $T_{3}^{\mu}$ and $T_{8}^{\mu}$ allow one to implement the gauge covariance of the fermion propagator [103]. Finally, as pointed out in Ref. [22] the contribution $T_{8}^{\mu}$ is able to generate an anomalous magnetic moment term, within a perturbative framework [21].

The adopted expressions of $\mathcal{F}_{3}$ and $\mathcal{F}_{8}$ are the ones given in [22] (see also [53]), where a very detailed formal analysis of $\Gamma_{T}^{\mu}$ was carried out (adopting a Euclidean metric) and general expression were found. In particular, due to the curl of the current, it turns out that $\mathcal{F}_{3}$ and $\mathcal{F}_{8}$ can be minimally chosen as linear combinations of $\mathcal{A}$ and $\mathcal{B}$ (see Sect. 2.1). In this way, one has a workable Ansätz for $\Gamma_{R ; T}^{\mu}$, that has the virtue of closing the equations involving the fermion and photon self-energies. The actual $\mathcal{F}_{3}$ and $\mathcal{F}_{8}$ are given by (see also Ref. [53])

$\mathcal{F}_{3}\left(p_{f}, p_{i}, \zeta\right)=-\frac{1}{2} F_{\mathcal{A}_{-}}\left(p_{f}, p_{i}, \zeta\right)$

$\mathcal{F}_{8}\left(p_{f}, p_{i}, \zeta\right)=F_{\mathcal{A}_{-}}\left(p_{f}, p_{i}, \zeta\right)$

that match the expected perturbative behavior for $p_{f}^{2}>>p_{i}^{2}$ (see the discussion in Ref. [53]). Hence, one can write

$$
\begin{aligned}
& \Gamma_{R ; T}^{\mu}\left(\zeta, p_{f}, p_{i}\right) \\
& =-\frac{1}{2}\left[q^{2} \gamma^{\mu}-q^{\mu} \phi+2 i \gamma_{5} \epsilon_{\nu \rho}^{\mu \alpha} \gamma_{\alpha} p_{i}^{v} q_{\rho}\right] F_{\mathcal{A}_{-}}\left(p_{f}, p_{i}, \zeta\right)
\end{aligned}
$$

In conclusion, we use $\Gamma_{R}^{\mu}$ given by the sum of the BallChiu vertex [50] and one of the minimal Ansätze for $\Gamma_{R ; T}^{\mu}$, proposed in Ref. [22]. In particular, by using Eq. (52) one can (i) fulfill the multiplicative renormalizability, (ii) establish a non-perturbative framework, where a closed coupled system of integral equations allows one to investigate the self-energies of both fermion and photon. 


\section{Coupled gap equations}

This section, in particular Sects. 4.1 and 4.2, contains the main outcomes of our formal elaboration that aims to get a mathematical tool for determining the fermion and photon NWFs and eventually yield the fermion and photon selfenergies. In order to accomplish such a task, it is necessary to proceed by writing down the DSEs for the self-energies (see, e.g., Ref. [6] for a general introduction), and insert the results obtained in Sect. 2 (see details in Appendix A and Appendix B).

The DSE for the regularized fermion self-energy, defined in Eq. (7), is given by

$$
\begin{aligned}
\Sigma_{Z}(\zeta, \Lambda ; p)= & -i Z_{1}(\zeta, \Lambda) e_{R}^{2} \int_{\Lambda} \frac{d^{4} k}{(2 \pi)^{4}} \\
& \times \gamma^{\beta} D_{\beta \alpha}^{R}(\zeta, p-k) S_{R}(\zeta, k) \Gamma_{R}^{\alpha}(\zeta ; k, p),
\end{aligned}
$$

where it is important to emphasize that the dependence upon $\Lambda$ means that the rhs can have singular contributions (indeed this is the case). But such terms become finite after introducing a suitable regularization procedure, that in our case it turns out to be the dimensional one with $d=4-\epsilon$ and $\Lambda=1 / \epsilon$ (see the details in Appendix A). Then, the renormalized self-energy fulfills

$$
\begin{aligned}
\Sigma_{R}(\zeta ; p)= & -i Z_{1}(\zeta, \Lambda) e_{R}^{2} \int_{\Lambda} \frac{d^{4} k}{(2 \pi)^{4}} \gamma^{\beta} S_{R}(\zeta, k) \\
& \times\left\{D_{\beta \alpha}^{R}(\zeta, p-k) \Gamma_{R}^{\alpha}(\zeta ; k, p)\right. \\
& \left.-\left[D_{\beta \alpha}^{R}(\zeta, p-k) \Gamma_{R}^{\alpha}(\zeta ; k, p)\right]_{p^{2}=\zeta^{2}}\right\}
\end{aligned}
$$

The two scalar functions describing $\Sigma_{R}(\zeta ; p)$ (see Eq. (2)) can be obtained by evaluating the suitable traces, i.e.

$$
\begin{aligned}
& \mathcal{A}_{R}(\zeta ; p)=\frac{1}{4 p^{2}} \operatorname{Tr}\left[p \Sigma_{R}(\zeta ; p)\right], \\
& \mathcal{B}_{R}(\zeta ; p)=\frac{1}{4} \operatorname{Tr}\left[\Sigma_{R}(\zeta ; p)\right]
\end{aligned}
$$

In Sect. 4.1 the results of the traces will be presented and the relation with the NWFs established.

In the Landau gauge we are adopting (recall that the polarization tensor is transverse in this gauge), one can start from the following expression of the regularized polarization tensor (see Appendix B for details) in terms of the renormalized quantities

$$
\begin{aligned}
& \Pi^{\mu \nu}(\zeta, \Lambda ; q) \\
& =-q^{2} T^{\mu \nu} \Pi(\zeta, \Lambda ; q)-i \frac{Z_{1}(\zeta, \Lambda)}{Z_{3}(\zeta, \Lambda)} e_{R}^{2} \int_{\Lambda} \frac{d^{4} k}{(2 \pi)^{4}} \\
& \quad \times \operatorname{Tr}\left\{\gamma^{\mu} S_{R}(\zeta, k) \Gamma_{R}^{v}(\zeta ; k, k-q) S_{R}(\zeta, k-q)\right\} .
\end{aligned}
$$

Notice that $T^{\mu \nu}$ is a symmetric tensor, and therefore also the rightmost term it has to be. One can convince himself by recalling that one has at disposal only one four-vector, $q^{\mu}$ for constructing antisymmetric contributions. It is understood that $\Pi^{\mu \nu}$, microscopically described by the second line in Eq. (56), must satisfy the transversity property, i.e $q_{\mu} \Pi^{\mu \nu}=$ $0, \quad \Pi^{\mu v} q_{v}=0$. Hence, one has to verify that

$$
\begin{aligned}
& \int_{\Lambda} \frac{d^{4} k}{(2 \pi)^{4}} \operatorname{Tr}\left\{q S_{R}(\zeta, k)\right. \\
& \left.\quad \times \Gamma_{R}^{v}(\zeta ; k, k-q) S_{R}(\zeta, k-q)\right\}=0, \\
& \int_{\Lambda} \frac{d^{4} k}{(2 \pi)^{4}} \operatorname{Tr}\left\{\gamma^{\mu} S_{R}(\zeta, k)\right. \\
& \left.\quad \times \Gamma_{R}(\zeta ; k, k-q) \cdot q S_{R}(\zeta, k-q)\right\}=0 .
\end{aligned}
$$

Since, we are adopting a vertex that automatically fulfills the WTI, the second line in Eq. (56) can be easily demonstrated by using the WTI itself and the dimensional regularization, in order to make formally allowed a shift in the integrand. As a matter of fact, one gets

$$
\begin{aligned}
& \int \frac{d^{d} k}{(2 \pi)^{4}} \operatorname{Tr}\left\{\gamma^{\mu} S_{R}(\zeta, k)\right. \\
& \left.\quad \times \Gamma_{R}(\zeta ; k, k-q) \cdot q S_{R}(\zeta, k-q)\right\} \\
& =\int \frac{d^{d} k}{(2 \pi)^{4}} \operatorname{Tr}\left\{\gamma^{\mu}\left[S_{R}(\zeta, k-q)-S_{R}(\zeta, k)\right]\right\}=0
\end{aligned}
$$

The equality in the first line of Eq. (57) is more involved, but for ensuring that the microscopic calculation of $\Pi^{\mu \nu}$ be proportional to $T^{\mu \nu}$ one should recover the structure

$g^{\mu v} A+\frac{q^{\mu} q^{v}}{q^{2}} B$

with the needed relations $A=-B$. This is guaranteed by Eq. (58), that follow from the fulfillment of WTI.

In order to single out the photon self-energy, $\Pi(\zeta, \Lambda ; q)$, one can proceed by saturating the polarization tensor with any combination of $g^{\mu \nu}$ and $q^{\mu} q^{\nu} / q^{2}$, but it is extremely useful to take full advantage and guidance from the analyses carried out in perturbative regime, (see, e.g., Refs. [6,42]). Hence, one can saturate both sides in Eq. (56) with the tensor $\mathcal{P}^{\mu \nu}$ given by

$\mathcal{P}^{\mu \nu}=g^{\mu \nu}-4 \frac{q^{\mu} q^{v}}{q^{2}}$.

This tensor has been introduced in previous works (see Refs. $[42,104,105])$ in order to project $T^{\mu \nu}$ on its $q^{\mu} q^{\nu}$ part, avoiding to deal with quadratic singularities proportional to $g^{\mu \nu}$ present in $\Pi^{\mu \nu}$. We emphasize that such a projector is adopted for convenience reasons. As a matter of fact, apparent quadratic singularities are met in the following elabo- 
ration, but the choice of the vertex presented in Sect. 3 (see also Appendix B) ensures their cancellations. These apparent singularities are easily bypassed by exploiting $\mathcal{P}^{\mu \nu}$, without carrying out a lengthy algebra (see also Refs. [104,105]). As a final remark, it should be pointed out that the formal manipulation shown in Appendix B needs a dimensional regularization of some terms and therefore one should substitute 4 with a generic dimension $d$ in the expression of $\mathcal{P}^{\mu \nu}$.

In conclusion, one gets the following expressions for the regularized self-energy

$$
\begin{aligned}
q^{2} & \Pi_{Z}(\zeta, \Lambda ; q)=-i \frac{Z_{1}(\zeta, \Lambda)}{3} e_{R}^{2} \int_{\Lambda} \frac{d^{4} k}{(2 \pi)^{4}} \mathcal{P}_{\mu \nu} \\
& \times \operatorname{Tr}\left[\gamma^{\mu} S_{R}(\zeta, k) \Gamma_{R}^{v}(\zeta, k, q) S_{R}(k-q)\right]
\end{aligned}
$$

where $\Pi_{Z}(\zeta, \Lambda ; q)=Z_{3}(\zeta, \Lambda) \Pi(\zeta, \Lambda ; q)$. This entails for the renormalized self-energy, Eq. (33),

$$
\begin{aligned}
& \Pi_{R}(\zeta ; q)=-i Z_{1}(\zeta, \Lambda) \frac{4}{3} e_{R}^{2} \int_{\Lambda} \frac{d^{4} k}{(2 \pi)^{4}} \mathcal{P}_{\mu \nu} \\
& \quad \times\left\{\frac{1}{4 q^{2}} \operatorname{Tr}\left[\gamma^{\mu} S_{R}(\zeta, k) \Gamma_{R}^{v}(\zeta, k, q) S_{R}(\zeta, k-q)\right]\right. \\
& \left.\quad-\frac{1}{4 \zeta_{p}^{2}} \operatorname{Tr}\left[\gamma^{\mu} S_{R}(\zeta, k) \Gamma_{R}^{v}(\zeta, k, q) S_{R}(\zeta, k-q)\right]_{q^{2}=\zeta_{p}^{2}}\right\}
\end{aligned}
$$

\subsection{The fermion gap equation and the NWFs}

As it is shown in details in Appendix A, one can exploit the NIR of $\mathcal{A}_{R}(\zeta ; p)$ and $\mathcal{B}_{R}(\zeta ; p)$, Eq. (55), and the KL representations of both fermion and photon propagators, Eqs. (15) and (39) respectively, for obtaining the following relations

$$
\begin{aligned}
\mathcal{A}_{R}(\zeta ; p) & =\int_{s_{t h}}^{\infty} d s \frac{\left(\zeta^{2}-p^{2}\right) \rho_{A}(s, \zeta)}{\left(p^{2}-s+i \epsilon\right)\left(\zeta^{2}-s+i \epsilon\right)} \\
& =\mathcal{T}_{A}(\zeta, \Lambda ; p)-\left.\mathcal{T}_{A}(\zeta, \Lambda ; p)\right|_{p^{2}=\zeta^{2}}
\end{aligned}
$$

and

$$
\begin{aligned}
\mathcal{B}_{R}(\zeta ; p) & =\int_{s_{t h}}^{\infty} d s \frac{\left(\zeta^{2}-p^{2}\right) \rho_{B}(s, \zeta)}{\left(p^{2}-s+i \epsilon\right)\left(\zeta^{2}-s+i \epsilon\right)} \\
& =\mathcal{T}_{B}(\zeta, \Lambda ; p)-\left.\mathcal{T}_{B}(\zeta, \Lambda ; p)\right|_{p^{2}=\zeta^{2}}
\end{aligned}
$$

where

$$
\begin{aligned}
& \mathcal{T}_{A}(\zeta, \Lambda ; p)=-i Z_{1}(\zeta, \Lambda) e_{R}^{2} \int_{0}^{\infty} d \omega \bar{\sigma}_{\gamma}\left(\omega, \zeta, \zeta_{p}^{2}\right) \\
& \quad \times \int_{0}^{\infty} d s^{\prime} \int_{\Lambda} \frac{d^{4} k}{(2 \pi)^{4}} \frac{1}{(p-k)^{2}-\omega+i \epsilon} \frac{1}{k^{2}-s^{\prime}+i \epsilon} \\
& \quad \times \frac{1}{4 p^{2}} \operatorname{Tr}\left\{\left[k \bar{\sigma}_{V}\left(s^{\prime}, \zeta, s_{t h}^{\prime}\right)+\bar{\sigma}_{S}\left(s^{\prime}, \zeta, s_{t h}^{\prime}\right)\right] \bar{\Gamma}_{T}^{\beta} p \gamma_{\beta}\right\},
\end{aligned}
$$

and

$$
\begin{aligned}
& \mathcal{T}_{B}(\zeta, \Lambda ; p)=-i Z_{1}(\zeta, \Lambda) e_{R}^{2} \int_{0}^{\infty} d \omega \bar{\sigma}_{\gamma}\left(\omega, \zeta, \zeta_{p}^{2}\right) \\
& \quad \times \int_{0}^{\infty} d s^{\prime} \int_{\Lambda} \frac{d^{4} k}{(2 \pi)^{4}} \frac{1}{(p-k)^{2}-\omega+i \epsilon} \frac{1}{k^{2}-s^{\prime}+i \epsilon} \\
& \quad \times \frac{1}{4} \operatorname{Tr}\left\{\left[\not k \bar{\sigma}_{V}\left(s^{\prime}, \zeta, s_{t h}^{\prime}\right)+\bar{\sigma}_{S}\left(s^{\prime}, \zeta, s_{t h}^{\prime}\right)\right] \bar{\Gamma}_{T}^{\beta} \gamma_{\beta}\right\} .
\end{aligned}
$$

In Eqs. (64) and (65), we have

$$
\begin{aligned}
& \bar{\sigma}_{\gamma}\left(\omega, \zeta, \zeta_{p}\right)=\delta\left(\omega-\zeta_{p}^{2}\right)+\sigma_{\gamma}(\omega, \zeta) \Theta\left(\omega-\zeta_{p}^{2}\right), \\
& \bar{\sigma}_{S(V)}\left(s^{\prime}, \zeta, s_{t h}^{\prime}\right)=\delta\left(s^{\prime}-m^{2}(\zeta)\right)+\sigma_{S(V)}\left(s^{\prime}, \zeta\right) \Theta\left(s^{\prime}-s_{t h}^{\prime}\right), \\
& \bar{\Gamma}_{T}^{\beta}=\frac{\gamma_{T}^{\beta}}{2} F_{\mathcal{A}_{+}}(k, p, \zeta)-\left((p+\not k) p_{T}^{\beta}+(p-k)^{2} \frac{\gamma_{T}^{\beta}}{2}\right. \\
& \left.\quad+i \gamma_{5} \epsilon^{\beta \alpha \nu \rho} \gamma_{\alpha} p_{v} k_{\rho}\right) F_{\mathcal{A}_{-}}(k, p, \zeta)-2 p_{T}^{\beta} F_{\mathcal{B}}(k, p, \zeta)
\end{aligned}
$$

with $\gamma_{T}^{\nu}=\gamma^{\nu}-q^{\nu} q \cdot \gamma / q^{2}$ and $p_{T}^{\mu}=p^{\mu}-q^{\mu} q \cdot p / q^{2}$.

In both $F_{\mathcal{A}_{+}}$and $F_{\mathcal{A}_{-}}$, a term $\mathcal{A}_{R}(\zeta ; p)$ is present. The one in $F_{\mathcal{A}_{+}}$generates a severe divergent behavior in $k$ (see Eqs. (64) and (65)) that cannot be regularized by subtraction, since the corresponding term in $\mathcal{T}_{A(B)}$, being evaluated at $p^{2}=\zeta^{2}$, yields $\mathcal{A}_{R}(\zeta ; \zeta)=0$, by definition. A simple power counting in $k^{2}$ reveals that in $\mathcal{T}_{A}$ and $\mathcal{T}_{B}$, only the combination proportional to $F_{\mathcal{A}_{+}}-\left(k^{2}-p^{2}\right) F_{\mathcal{A}_{-}}$allows one to mitigate the divergent behavior due to $\mathcal{A}_{R}(\zeta ; p)$ in $F_{\mathcal{A}_{+}}$, leading to a logarithmic divergence that can be regularized by the subtraction in Eqs.(62) and (63) (see details in Appendix A). In fact, one has

$$
F_{\mathcal{A}_{+}}(k, p, \zeta)-\left(k^{2}-p^{2}\right) F_{\mathcal{A}_{-}}(k, p, \zeta)=2\left(1-\mathcal{A}_{R}(\zeta ; k)\right)
$$

This cancellation highlights the intrinsic limitation of the $\mathrm{BC}$ vertex, since it is necessary to go beyond such a contribution for restoring the multiplicative renormalizability. This has been known from a long time (see, e.g. Ref. [52]), but it is relatively more recent the suggestion that the constraints coming from the curl of the vertex allow one to elaborate transverse contributions suitable for ensuring the multiplicative renormalizability (see, e.g., Ref. [22]). In Appendix A it is explicitly shown how non-multiplicatively renormalizable contributions, from the BC term, Eq. (46), and the transverse ones, Eq. (52), cancel each other.

Once the explicit expressions of the relations in Eqs. (62) and (63), are obtained as in Eqs. (A.63) and (A.50), respectively, by using a spacelike external momentum $p$ in order to avoid unnecessary formal complexities (recall that the NWFs are real functions that do not depend upon the external momenta as one can also assess a posteriori), one can 
extract the integral equations fulfilled by the corresponding NWFs $\rho_{A}$ and $\rho_{B}$, after assuming that the uniqueness theorem by Nakanishi [57] can be applicable to the nonperturbative regime.

In particular comparing Eq. (A.63) and the lhs of Eq. (62), one gets the desired relation for $\rho_{A}$ (see Appendix A)

$$
\begin{aligned}
& \Theta\left(y-s_{t h}\right) \rho_{A}(y, \zeta)=\frac{3}{(4 \pi)^{2}} e_{R}^{2} \lim _{\Lambda \rightarrow \infty} Z_{1}(\zeta, \Lambda) \\
& \quad \times \int_{0}^{\infty} d \omega \bar{\sigma}_{\gamma}(\omega, \zeta, \zeta p, \Lambda) \int_{0}^{1} d \xi \int_{0}^{\infty} d s^{\prime} \\
& \quad \times\left\{\overline { \sigma } _ { V } ( s ^ { \prime } , \zeta , s _ { t h } ^ { \prime } , \Lambda ) \left[\xi \Theta\left(y \xi(1-\xi)-\xi \omega-(1-\xi) s^{\prime}\right)\right.\right. \\
& \left.\quad-\int_{0}^{1-\xi} d t \Theta\left(y t(1-t)-\xi \omega-t s^{\prime}\right)\right]+\bar{\sigma}_{V}\left(s^{\prime}, \zeta, s_{t h}^{\prime}, \Lambda\right) \\
& \quad \times\left[\int_{s_{t h}}^{\infty} d s \rho_{A}(s, \zeta, \Lambda) \mathcal{C}_{A V}^{(0)}\left(\zeta, \omega, s, s^{\prime}, \xi, y\right)\right. \\
& \left.\quad+y \int_{s_{t h}}^{\infty} d s \rho_{A}(s, \zeta, \Lambda) \mathcal{C}_{A V}^{(1)}\left(\zeta, \omega, s, s^{\prime}, \xi, y\right)\right] \\
& \quad-y \bar{\sigma}_{S}\left(s^{\prime}, \zeta, s_{t h}^{\prime}, \Lambda\right) \int_{0}^{1-\xi} d t \int_{0}^{1-\xi-t} d w \\
& \quad \times \int_{s_{t h}}^{\infty} d s \rho_{B}(s, \zeta, \Lambda) \\
& \quad \times \Delta^{\prime}\left[y-s+\frac{s \mathcal{A}_{4}(t, w)-\xi \omega-t s^{\prime}-w s}{\mathcal{A}_{4}(t, w)}\right.
\end{aligned}
$$

with $\mathcal{A}_{4}(t, w)=(t+w)(1-t-w)$,

$$
\begin{aligned}
& \Delta^{\prime} {[y-s+(s A-B) / A] } \\
&=\frac{\delta[y-s+(s A-B) / A]-\delta(y-s)}{(s A-B)}, \\
& \mathcal{C}_{A V}^{(0)}\left(\zeta, \omega, s, s^{\prime}, \xi, y\right)=\frac{1}{\left(\zeta^{2}-s+i \epsilon\right)} \\
& \quad \times\left\{\xi \Theta\left[y \xi(1-\xi)-\xi \omega-(1-\xi) s^{\prime}\right]\right. \\
&\left.-\int_{0}^{1-\xi} d t \Theta\left[y t(1-t)-\xi \omega-t s^{\prime}\right]\right\} \\
&+\int_{0}^{1-\xi} d t\left\{\frac{1}{(1-\xi)} \delta\left[y-\frac{\xi \omega+t s^{\prime}+(1-\xi-t) s}{\xi(1-\xi)}\right]\right. \\
&\left.-\int_{0}^{1-\xi-t} \frac{d w}{\mathcal{A}_{4}(t, w)} \delta\left[y-\frac{\xi \omega+t s^{\prime}+w s}{\mathcal{A}_{4}(t, w)}\right]\right\}
\end{aligned}
$$

and

$$
\begin{aligned}
& \mathcal{C}_{A V}^{(1)}\left(\zeta, \omega, s, s^{\prime}, \xi, y\right)=\int_{0}^{1-\xi} d t\{(1+\xi) \xi \\
& \quad \times \Delta^{\prime}\left[y-s+\frac{s \xi(1-\xi)-\xi \omega-t s^{\prime}-(1-\xi-t) s}{\xi(1-\xi)}\right]
\end{aligned}
$$

$$
\left.-\int_{0}^{1-\xi-t} d w \Delta^{\prime}\left[y-s+\frac{s \mathcal{A}_{4}(t, w)-\xi \omega-t s^{\prime}-w s}{\mathcal{A}_{4}(t, w)}\right]\right\} .
$$

It has to be pointed out that the presence of the function $\Delta^{\prime}$ does not prevent a quantitative investigation (to be presented elsewhere) once an integration with a function smooth enough is carried out. Indeed, the existence of NWFs fulfilling a suitable smoothness property will be the target of future numerical investigations. In the meanwhile, we should consider as an encouraging hint the results of the first quantitative check discussed in Sect. 5 since the obtained first-order NWFs lead to well-defined integrals in Eq. (68) when iterating further.

As to $\rho_{B}$, after comparing Eq. (A.50) and the lhs of Eq. (63), one extracts

$$
\begin{aligned}
\Theta & \left(y-s_{t h}\right) \rho_{B}(y, \zeta)=-\frac{3}{(4 \pi)^{2}} e_{R}^{2} \lim _{\Lambda \rightarrow \infty} Z_{1}(\zeta, \Lambda) \\
& \times \int_{0}^{\infty} d \omega \bar{\sigma}_{\gamma}\left(\omega, \zeta, \zeta_{p}, \Lambda\right) \int_{0}^{1} d \xi \int_{0}^{\infty} d s^{\prime} \\
& \times\left\{\bar{\sigma}_{S}\left(s^{\prime}, \zeta, s_{t h}^{\prime}, \Lambda\right) \Theta\left[y \xi(1-\xi)-\xi \omega-(1-\xi) s^{\prime}\right]\right. \\
& +\bar{\sigma}_{S}\left(s^{\prime}, \zeta, s_{t h}^{\prime}, \Lambda\right) \\
& \times\left[\int_{s_{t h}}^{\infty} d s \rho_{A}(s, \zeta, \Lambda) \mathcal{C}_{A S}^{(0)}\left(\zeta, \omega, s, s^{\prime}, \xi, y\right)\right. \\
& \left.+y \int_{s_{t h}}^{\infty} d s \rho_{A}(s, \zeta, \Lambda) \mathcal{C}_{A S}^{(1)}\left(\zeta, \omega, s, s^{\prime}, \xi, y\right)\right] \\
& +y \bar{\sigma}_{V}\left(s^{\prime}, \zeta, s_{t h}^{\prime}, \Lambda\right) \int_{0}^{1-\xi} d t \int_{0}^{1-\xi-t} d w \\
& \times \int_{s_{t h}}^{\infty} d s \rho_{B}(s, \zeta, \Lambda) \\
& \left.\times \Delta^{\prime}\left[y-s+\frac{s \mathcal{A}_{4}(t, w)-\xi \omega-t s^{\prime}-w s}{\mathcal{A}_{4}(t, w)}\right]\right\}
\end{aligned}
$$

with

$$
\begin{aligned}
& \mathcal{C}_{A S}^{(0)}\left(\zeta, \omega, s, s^{\prime}, \xi, y\right)=\frac{1}{\zeta^{2}-s+i \epsilon} \\
& \times \Theta\left[y \xi(1-\xi)-\xi \omega-(1-\xi) s^{\prime}\right]+\frac{1}{\xi(1-\xi)} \\
& \quad \times \int_{0}^{1-\xi} d z \delta\left[y-\frac{\xi \omega+z s^{\prime}+(1-\xi-z) s}{\xi(1-\xi)}\right],
\end{aligned}
$$

and

$$
\begin{aligned}
& \mathcal{C}_{A S}^{(1)}\left(\zeta, \omega, s, s^{\prime}, \xi, y\right)=\frac{1}{\zeta^{2}-s+i \epsilon} \int_{0}^{1-\xi} d t\{(1-\xi) \\
& \quad \times \Delta^{\prime}\left[y-s+\frac{s \xi(1-\xi)-\xi \omega-t s^{\prime}-(1-\xi-t) s}{\xi(1-\xi)}\right] \\
& \quad+\int_{0}^{1-\xi-t} d w
\end{aligned}
$$




$$
\left.\times \Delta^{\prime}\left[y-s+\frac{s \mathcal{A}_{4}(t, w)-\xi \omega-t s^{\prime}-w s}{\mathcal{A}_{4}(t, w)}\right]\right\} .
$$

\subsection{The photon gap equation and the NWF}

In Appendix B, the details are given for obtaining the integral equation fulfilled by the NWF $\rho_{\gamma}$ (see Eq. (38)), exploiting both the integral equation that determines the renormalized photon self-energy, Eq. (61) and the uniqueness theorem [57]. One can write

$$
\begin{aligned}
\Pi_{R}(\zeta ; q) & =\int_{\zeta_{p}^{2}}^{\infty} d s \frac{\left(\zeta_{p}^{2}-q^{2}\right) \rho_{\gamma}(s, \zeta)}{\left(\zeta_{p}^{2}-s+i \epsilon\right)\left(q^{2}-s+i \epsilon\right)} \\
& =\left[\mathcal{T}_{P}(\zeta, \Lambda ; q)-\left.\mathcal{T}_{P}(\zeta, \Lambda ; q)\right|_{q^{2}=\zeta_{p}^{2}}\right]
\end{aligned}
$$

where

$$
\begin{aligned}
\mathcal{T}_{P}(\zeta, \Lambda ; q)= & -i Z_{1}(\zeta, \Lambda) \frac{4}{3} e_{R}^{2} \int_{\Lambda} \frac{d^{4} k}{(2 \pi)^{4}} \mathcal{P}_{\mu \nu} \frac{1}{4 q^{2}} \\
& \times \operatorname{Tr}\left[\gamma^{\mu} S_{R}(\zeta, k) \Gamma_{R}^{\nu}(\zeta, k, q) S_{R}(\zeta, k-q)\right] .
\end{aligned}
$$

Then, following the formal steps in Appendix B, where a spacelike $q^{2}$ has been adopted for a straightforward elaboration without loss of generality (as in the fermionic case), one gets

$$
\begin{aligned}
\Theta & \left.y-s_{t h}^{p}\right) \rho_{\gamma}(y, \zeta)=-\frac{e_{R}^{2}}{(2 \pi)^{2}} \lim _{\Lambda \rightarrow \infty} Z_{1}(\zeta, \Lambda) \int_{0}^{\infty} d s \\
& \times \int_{0}^{\infty} d s^{\prime} \int_{0}^{1} d \xi\left\{\bar{\sigma}_{V}\left(s^{\prime}, \zeta, s_{t h}^{\prime}, \Lambda\right) \bar{\sigma}_{V}\left(s, \zeta, s_{t h}, \Lambda\right)\right. \\
& \left.\times 2 \xi(1-\xi) \Theta\left[y \xi(1-\xi)-\xi s^{\prime}-(1-\xi) s\right)\right] \\
& \times\left(1+\int_{s_{t h}}^{\infty} d \omega \frac{\rho_{A}(\omega, \zeta, \Lambda)}{\left(\zeta^{2}-\omega+i \epsilon\right)}\right) \\
& +\int_{s_{t h}}^{\infty} d \omega \rho_{A}(\omega, \zeta, \Lambda) \mathcal{C}_{\gamma}\left(s, s^{\prime}, \xi, \omega\right) \\
& +2 \bar{\sigma}_{S}\left(s^{\prime}, \zeta, s_{t h}^{\prime}, \Lambda\right) \bar{\sigma}_{V}\left(s, \zeta, s_{t h}, \Lambda\right) \\
& \times \int_{s_{t h}}^{\infty} d \omega \rho_{B}(\omega, \zeta, \Lambda) \int_{0}^{1-\xi} d v \int_{0}^{1-\xi-v} d w(v+w) \\
& \left.\times \frac{1-2(v+w)}{\mathcal{A}_{4}^{2}(v, w)} \frac{\partial}{\partial y} \delta\left[y-\mathcal{A}_{7}\left(s, s^{\prime}, \omega, v, \xi, w\right)\right]\right\},(77)
\end{aligned}
$$

with

$$
\begin{aligned}
& \mathcal{A}_{7}\left(s, s^{\prime}, \omega, v, \xi, w\right) \\
& =\frac{v s^{\prime}+(\xi+w) \omega+(1-\xi-v-w) s}{(v+w)(1-v-w)} \\
& \mathcal{C}_{\gamma}\left(s, s^{\prime}, \xi, \omega\right)=\int_{0}^{1-\xi} d v \int_{0}^{1-\xi-v} \frac{d w}{\mathcal{A}_{4}(v, w)} \\
& \quad \times\left\{2 \bar{\sigma}_{V}\left(s^{\prime}, \zeta, s_{t h}^{\prime}\right) \bar{\sigma}_{V}\left(s, \zeta, s_{t h}\right)\right.
\end{aligned}
$$

$$
\begin{aligned}
& \times\left(\omega \frac{\partial}{\partial y} \delta\left[y-\mathcal{A}_{7}\left(s, s^{\prime}, \omega, v, \xi, w\right)\right]\right. \\
& \left.+\delta\left[y-\mathcal{A}_{7}\left(s, s^{\prime}, \omega, v, \xi, w\right)\right]\right) \\
& +\bar{\sigma}_{S}\left(s^{\prime}, \zeta, s_{t h}^{\prime}\right) \bar{\sigma}_{S}\left(s, s_{t h}^{\prime}, \zeta\right) \\
& \left.\times \frac{\partial}{\partial y} \delta\left[y-\mathcal{A}_{7}\left(s, s^{\prime}, \omega, v, \xi, w\right)\right]\right\},
\end{aligned}
$$

and $\mathcal{A}_{4}(v, w)=(t+w)(1-t-w)$. In order to obtain numerical solutions, the derivatives of the Dirac $\delta$ can be traded for the derivative of the $\rho$ functions, which, contrary to the $\bar{\sigma}$, does not contain itself any Dirac $\delta$. Just like in the fermion case, the first check performed in Sect. 5 yields results compatible with well defined integrals in (77). One can also note that in the right-hand side of Eq. (77), the dependence upon $\rho_{\gamma}$ is not explicit, but buried into the fermionic quantities.

\subsection{Some remarks}

Concluding this section, that presents our formal results, some remarks are in order. The coupled systems for determining $\rho_{A}, \rho_{B}$ and $\rho_{\gamma}$ is composed by the set of Eqs. (68), (72) and (77), supplemented with Eqs. (23) and (43). The fact that the NWFs do not depend on external momenta has been extensively used to build the system of equations. Indeed, Eqs. (23) and (43) are obtained from timelike (above threshold) momenta, taking advantage of the real and imaginary part decomposition, while Eqs. (68), (72) and (77) are derived for spacelike external momenta to avoid complications coming from singularities. Beside the above feature, the uniqueness theorem allows one to finalize the formal steps. Interestingly, derivatives of Dirac delta distribution naturally appear in our derivations. In summary, (i) the NWFs are real functions that do not depend upon the external momentum, as a posteriori can be checked by a direct inspection of the coupled system; (ii) once the NWFs are numerically evaluated, the scalar functions $\mathcal{A}_{R}(\zeta ; p), \mathcal{B}_{R}(\zeta ; p)$ and $\Pi_{R}(\zeta ; q)$ are known for any value of any momenta; (iii) the presence of the derivative of the delta-function is not an issue from the numerical point of view, as already observed when the NIR approach has been applied to the numerical solution of BSE (see, e.g., Refs. [70,72]).

\section{A first application}

After establishing the formal results, i.e. the system of integral equations that the NWFs $\rho_{A}, \rho_{B}$ and $\rho_{\gamma}$ have to fulfill, it is important to test the consistency. Following the same spirit of the first applications of the NIR approach to the two-scalar system, where the Wick-Cutokski model had to stem from the formal elaboration (see, e.g., Refs. [106, 107]), 
we have performed the first iteration of the coupled system, as an initial step, in view of the quantitative investigation in full, to be presented elsewhere. It should be pointed out that the first iteration offers the possibility to partly check our results, as it corresponds to the standard one-loop computation. Once the analytical expressions of $\rho_{A}^{(1)}, \rho_{B}^{(1)}$ and $\rho_{\gamma}^{(1)}$ have been obtained, we have carried out the evaluations of (i) the KL weights for both fermion and photon propagators, (ii) the fermionic running mass and iii) the charge renormalization function, and eventually compared with one-loop results (see, e.g., Ref. [89], but noting the different renormalization scheme).

The numerical investigation, aimed at establishing the validity of our approach by assessing the convergence of the iterative method, will be presented elsewhere. We stress that, generally speaking, the result of the iterative procedure may differ significantly from the first iteration one (see, e.g., Refs. [108,109] and Ref. [110] for QCD studies), and that we perform here a basic test of consistency. In Appendix C all the details for obtaining the aforementioned first iteration, Eqs. (C.116), (C.120) and (C.122) respectively, are illustrated with also some of their features, while in Appendix D, the full expressions of the coupled system is summarized for the convenience of the interested reader.

In Fig. 2, the first-order Källén-Lehman weights for the fermionic propagator, Eq. (15), are presented for different values of the IR regulator $\zeta_{p}$. They can be easily obtained from Eq. (23) after inserting $\rho_{A}^{(1)}$ and $\rho_{B}^{(1)}$ given by (see Eqs. (C.116), with its careful discussion, and (C.120))

$$
\begin{aligned}
& \Theta\left(y-s_{t h}\right) \rho_{A}^{(1)}(y, \zeta)=-\frac{e_{R}^{2}}{2(4 \pi)^{2}} \frac{1}{\zeta_{p}^{2} y^{2}} \\
& \quad \times \Theta\left(y-m^{2}(\zeta)\right)\left\{\Theta\left[\left[m(\zeta)+\zeta_{p}\right]^{2}-y\right]\left(y-m^{2}(\zeta)\right)^{3}\right. \\
& \quad+\Theta\left[y-\left[m(\zeta)+\zeta_{p}\right]^{2}\right]\left(y-m^{2}(\zeta)\right)^{3} \\
& \left.\quad \times\left[1-f\left(y, \zeta, \zeta_{p}^{2}\right)\right]\right\}
\end{aligned}
$$

with

$$
\begin{aligned}
f\left(y, \zeta^{2}, \zeta_{p}^{2}\right)= & \sqrt{1-\zeta_{p}^{2} \frac{2 y+2 m^{2}(\zeta)-\zeta_{p}^{2}}{\left(y-m^{2}(\zeta)\right)^{2}}} \\
& \times\left[1+\zeta_{p}^{2} \frac{y+m^{2}(\zeta)-2 \zeta_{p}^{2}}{\left(y-m^{2}(\zeta)\right)^{2}}\right]
\end{aligned}
$$

and

$$
\begin{aligned}
& \Theta\left(y-s_{t h}\right) \rho_{B}^{(1)}(y, \zeta) \\
& \quad=-\frac{3 e_{R}^{2}}{(4 \pi)^{2}} m(\zeta) \Theta\left[y-\left[m(\zeta)+\zeta_{p}\right]^{2}\right]
\end{aligned}
$$

$$
\times \frac{1}{y} \sqrt{\left[y-m^{2}(\zeta)-\zeta_{p}^{2}\right]^{2}-4 m^{2}(\zeta) \zeta_{p}^{2}}
$$

Qualitatively $\sigma_{V}$ and $\sigma_{S}$ are quite similar, though width and tail of $\sigma_{S}$ are slightly larger than the $\sigma_{V}$ ones. The common features are (i) the negative values (in Ref. [44] the scalar weight is also negative), that is a consequence of our choice of the renormalization scheme, and (ii) the sharp peaks for $\zeta_{p} \rightarrow 0$. The latter are very close to the threshold and clearly depend upon the IR-regulator $\zeta_{p}$, while the tails are unaffected, as expected. To complete the discussion it is useful to recall that the residue at the pole of the renormalized fermion propagator, $\mathcal{R}_{S}$, is not equal to 1 , as expected in the RI'/MOM scheme [96,97]. Table 1 summarizes the different values of the residues associated with our curves in Fig. 2.

Another simple check is the formal comparison between the expression of $\rho_{B}^{(1)}(y, \zeta)$ for vanishing values of $\zeta_{p}$,

Table 1 Values of the fermion propagator residue $\mathcal{R}_{S}$ for different $\zeta_{p}$

\begin{tabular}{lllll}
\hline $100 \zeta_{p} / m$ & 4 & 3 & 2 & 1 \\
\hline $\mathcal{R}_{S}$ & 1.10 & 1.11 & 1.13 & 1.16 \\
\hline
\end{tabular}
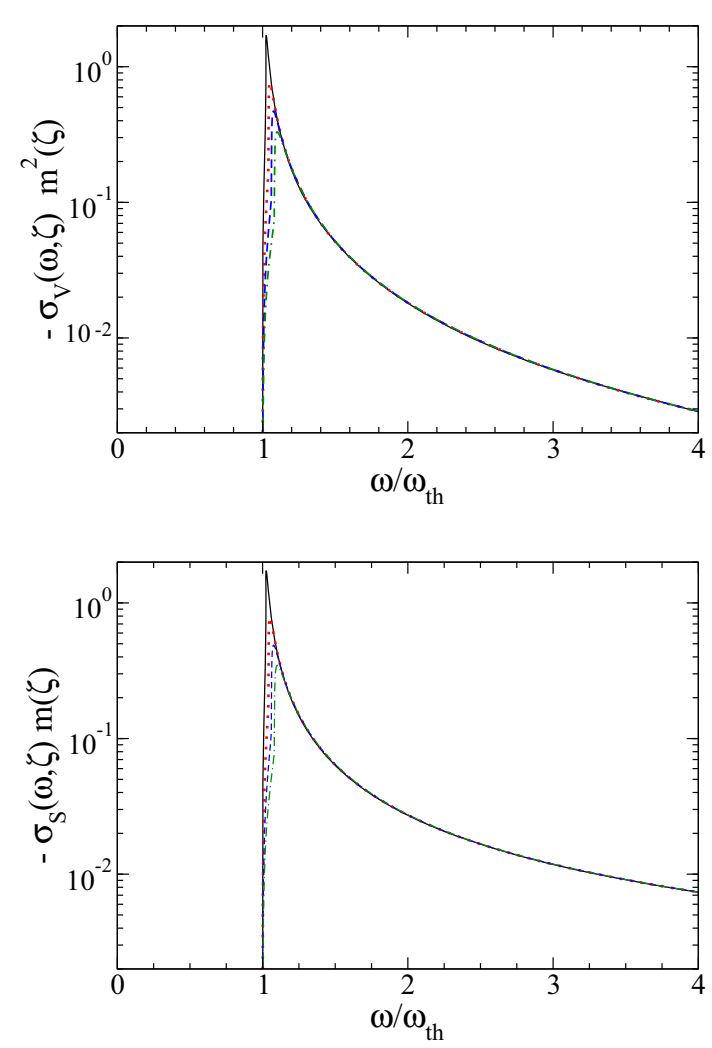

Fig. 2 The first iteration of the Källén-Lehman weights for the fermionic propagator, Eq. (15), for different values of the IR-regulator, $\zeta_{p}$. The threshold is given by $\omega_{t h}=m^{2}(\zeta)$. Upper panel the vector weight, $\sigma_{V}(\omega, \zeta)$. Lower panel: scalar weight $\sigma_{S}(\omega, \zeta)$. Solid line: $\zeta_{p}=0.01 m(\zeta)$. Dotted line: $\zeta_{p}=0.02 m(\zeta)$. Dashed line: $\zeta_{p}=0.03 \mathrm{~m}(\zeta)$. Dash-dotted line: $\zeta_{p}=0.04 \mathrm{~m}(\zeta)$ 
with the expression one can extract from standard one-loop computations of $\mathcal{B}_{R}(\zeta ; p)$ (see e.g. [89]), but within the RI'/MOM scheme. In the limit $\zeta_{p} \rightarrow 0$, one has from Eq. (81)

$$
\begin{aligned}
\lim _{\zeta_{p} \rightarrow 0} \Theta\left(y-s_{t h}\right) \rho_{B}^{(1)}(y, \zeta)= & -3 \frac{\alpha_{e m}}{4 \pi} \Theta\left(y-m^{2}(\zeta)\right) \\
& \times m(\zeta)\left[1-\frac{m^{2}(\zeta)}{y}\right]
\end{aligned}
$$

with $\alpha_{e m}=e_{R}^{2} / 4 \pi$. Moreover from the definition in Eq. (14) one writes

$\Im m\left\{\mathcal{B}_{R}(\zeta, p)\right\}=-\pi \Theta\left(p^{2}-s_{t h}\right) \rho_{B}^{(1)}\left(p^{2}, \zeta\right)$.

After imposing $\mathcal{B}_{R}(\zeta, \zeta)=0$, perturbative computations yield (see e.g. [89])

$$
\begin{aligned}
\mathcal{B}_{R}^{1 \text {-loop }}(\zeta, p)= & m(\zeta) \frac{\alpha_{\text {em }}}{4 \pi} 3 \frac{m^{2}-p^{2}}{p^{2}} \\
& \times \ln \left(1-\frac{p^{2}}{m^{2}(\zeta)}\right),
\end{aligned}
$$

Hence, for $p^{2}>m^{2}(\zeta)$ the logarithm becomes complex, and adopting the same analytic continuation as in Ref. [89] (i.e. $\ln (-\rho)=\ln \rho-i \pi)$, eventually one has

$\Im m\left\{\mathcal{B}_{R}^{I Z}(\zeta, p\}=\pi m(\zeta) 3 \frac{\alpha_{e m}}{4 \pi}\left[1-\frac{m^{2}(\zeta)}{p^{2}}\right]\right.$.

that coincides with the result one gets from Eqs. (81) and (83). As a final remark, one should point out that in the same limit $\mathcal{A}_{R}(\zeta, p)$ vanishes both in our case (see Eq. (C.119)) as well as in Ref. [89].

Figure 3 shows the Källén-Lehman weight for the photon propagator, Eq. (43), obtained from $\rho_{\gamma}^{(1)}$ given by (see also

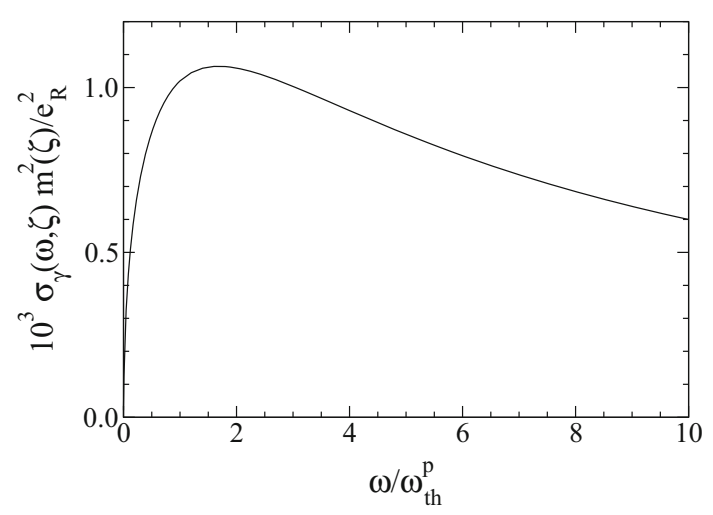

Fig. 3 The first iteration of the Källén-Lehman weight for the photon propagator, Eq. (39). N.B. in this case there is no dependence upon the IR-regulator, $\zeta_{p}$, as shown in Eq. (86). The threshold is given by $\omega_{t h}^{p}=[2 m(\zeta)]^{2}$
Eq. (C.122))

$$
\begin{aligned}
\Theta\left(y-s_{t h}^{p}\right) \rho_{\gamma}^{(1)}(y, \zeta)= & -\frac{e_{R}^{2}}{3(2 \pi)^{2}} \Theta(y) \Theta\left(y-4 m^{2}(\zeta)\right) \\
& \times\left(1+2 \frac{m^{2}(\zeta)}{y}\right) \sqrt{1-4 \frac{m^{2}(\zeta)}{y}}
\end{aligned}
$$

The independence from the IR-regulator $\zeta_{p}$, as shown in the expression of $\rho_{\gamma}^{(1)}$, is the standard feature of the one-loop calculation, and only the higher-order contributions will make apparent such a dependence. Differently from the fermion case, the KL weight of the photon is positive, as expected. This bosonic result points to the highly non trivial interplay of the two scalar functions $\mathcal{A}_{R}$ and $\mathcal{B}_{R}$, in order to obtain positive KL weights for the fermionic source. We have also calculated the running mass (see Eq. (4) for the value at the renormalization point)

$$
\begin{aligned}
M(\zeta ; p) & =\frac{m(\zeta)+\mathcal{B}_{R}(\zeta ; p)}{1-\mathcal{A}_{R}(\zeta ; p)} \\
& =m(\zeta)+\frac{m(\zeta) \mathcal{A}_{R}(\zeta ; p)+\mathcal{B}_{R}(\zeta ; p)}{1-\mathcal{A}_{R}(\zeta ; p)}
\end{aligned}
$$

and the charge renormalization function (Eq. (29) for the value at the renormalization point)

$$
G\left(\zeta ; q^{2}\right)=\frac{\alpha_{R}\left(\zeta ; q^{2}\right)}{\alpha_{e m}}=\frac{1}{1+\Pi_{R}(\zeta ; q)} .
$$

In Fig. 4, the running mass is shown for values of the four-momentum below the threshold, $s_{t h}=m^{2}(\zeta)$, adopting a tiny $\zeta_{p}$ up to $\zeta_{p} / m(\zeta)=10^{-4}$, while in Fig. 5 both real and imaginary terms, generated for $p^{2} \geq s_{t h}$, are presented. Notice that the positive sign of the imaginary part is a consequence of the first-order calculation (see. Eq. (85) and the vanishing of $\mathcal{A}_{R}$ for $\zeta_{p} \rightarrow 0$ ).

In Figs. 6 and 7, the running charge defined in Eq. (88) is shown for values below and above the threshold, that in this

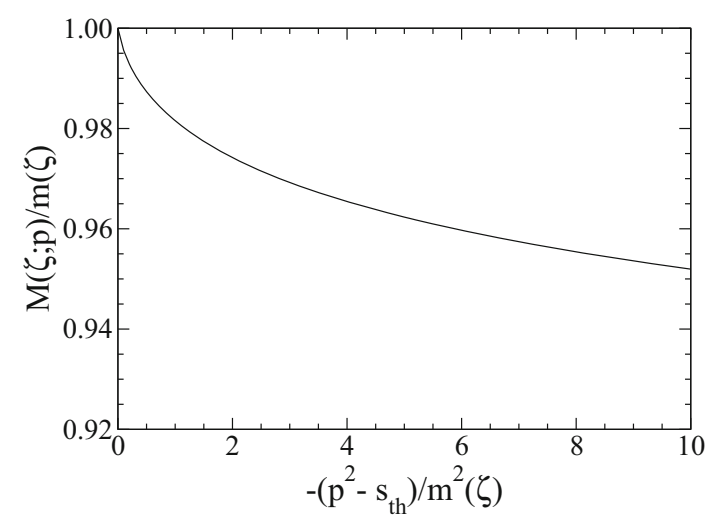

Fig. 4 The invariant mass $M(\zeta ; p)$, Eq. (87), below the threshold $s_{t h}=$ $m(\zeta)^{2}$, vs $p^{2}$ 

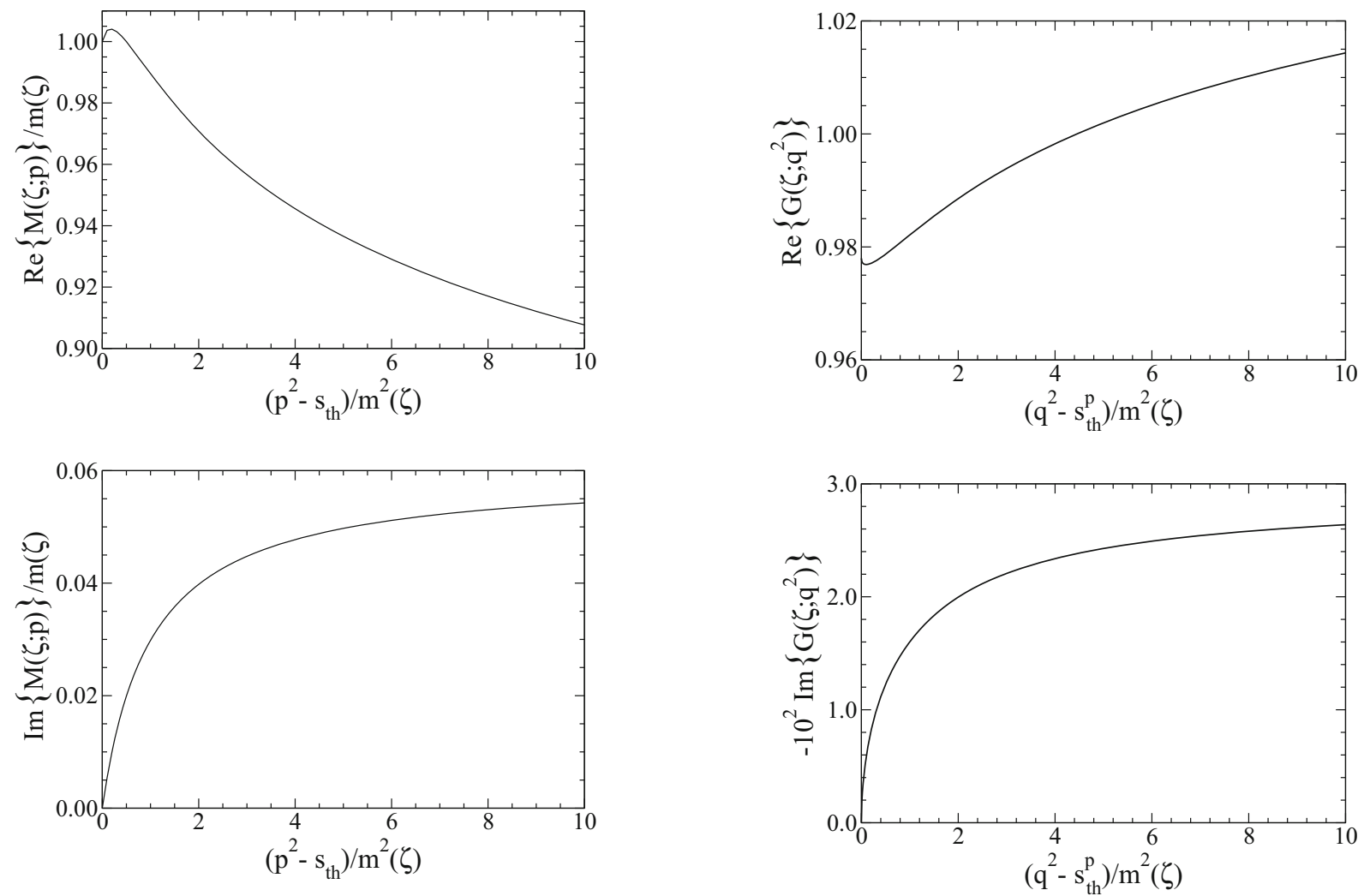

Fig. 5 The same as in Fig. 4, but in the timelike region. Upper panel: real part of the running mass. Lower panel: imaginary part of the running mass

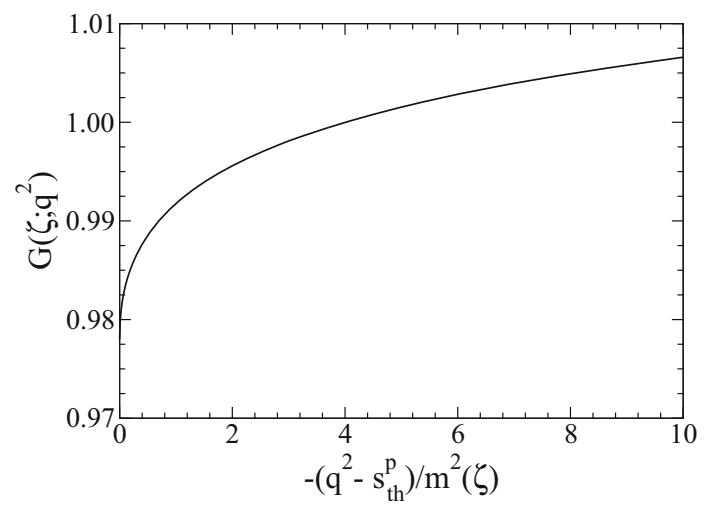

Fig. 6 The running charge $G\left(\zeta ; q^{2}\right)$, Eq. (88), below the threshold vs $q^{2}$, with $s_{t h}^{p}=4 m^{2}(\zeta)$

case holds $s_{t h}^{p}=4 m^{2}(\zeta)$. The comparison with the results of Ref. [61] (where $\Im m\{M(\zeta, p)\}$ has a negative sign) can be be performed only at the qualitative level, since the quantitative one is too early for our numerical efforts. In any case, one can recognize quite similar pattern, in particular, for the case of small values of the coupling constant $\alpha_{e m}$. It should be pointed out that the first-order self-energies (both fermion and photon ones) depend linearly upon the coupling constant

Fig. 7 The same as in Fig. 6, but for the timelike region. Upper panel: the real part. Lower panel: the imaginary part

and therefore, the values of the running mass and the relative running charge are not affected by such a dependence.

We would like to conclude this section by shortly anticipating a remark stemming from the detailed numerical investigations to be presented elsewhere. Indeed, in the quenched approximation, one can verify that the integrals relevant for the second iteration are all converging, even the ones including the function $\Delta^{\prime}$ (see Eq. (69)). Of course, when extending the calculations to large values of the coupling constant, extra care on the convergence of the above quantities is requested, as one should expect additional singularity issues from Minkowski and Euclidean calculations focusing on the strong-coupling regime, both below and above the critical value (see, e.g., Refs. [47,61,94,111] for the challenges beyond the critical value).

\section{Conclusions and perspectives}

This work belongs to the set of the early attempts (not too much numerous) to explore the non-perturbative regime of $Q E D_{3+1}$ directly in Minkowski space, by exploiting the framework based on the so-called Nakanishi integral rep- 
resentation for describing the self-energies of both fermion and photon.

The originality of this work, elaborated within the RI'/MOM scheme, lies in the choice of the fermion-photon vertex, able to fulfill constraints coming from both the Ward-Takashi identity and the multiplicative renormalizability, that calls for purely transverse contributions. We have shown that despite the apparent complexity, it is possible to derive a well-defined system of equations for the Nakanishi weight functions, that we recall are real functions fulfilling a uniqueness theorem, within the Feynman diagrammatic framework [57]. In addition, we have presented an initial check based on the evaluation of the first iteration of the coupled system. In particular, we have initiated the comparison with known results of (i) the Källén-Lehmann weights for both fermion and photon, (ii) the running mass and (iii) the charge renormalization function. Beyond this, we have also verified that numerical stability remains under control, encouraging toward a more vast numerical investigation.

It has to be pointed out that the present results readily calls for two natural extensions on a short-time scale. First, complete numerical studies should be performed, allowing one to assess the convergence of the whole approach and to move the comparison to a quantitative level, e.g. with the results in Refs. [44,61]. Second, the expected residue equal to one at the mass pole should be implemented at the level of the NWFs, i.e. going from the RI'/MOM scheme to the standard on-shell renormalization scheme.

On a longer time-scale, the third desirable extension would be to move from QED to QCD. An educated reader might object that many ingredients we used are not available or available in a much more complicated way for QCD. For instance, there is no formal proof that the propagators of confined particles should have Källén-Lehmann-like representation (positive definite). Nonetheless, lattice-QCD computations seem to be consistent with a spectral representation (although not a positive one) [112]. Furthermore, the WardTakahashi identities must be replaced by the Slavnov-Taylor ones, forcing deep modifications of the quark-gluon vertex function, playing an important role in realizing a dynamical breakdown of chiral symmetry. Also for this issue, progresses have been recently done in that direction, with the definition of the non-Abelian generalization of the Ball-Chiu vertex [27]. Therefore, despite the technical difficulties to jump from QED to QCD, we believe that such a possibility should deserve a careful investigation.

As a final remark, it is appropriate to recall that, for a given interacting system, our final goal is to implement and solve the BSE with dressed propagators for both particles and quanta, directly in Minkowski space, i.e. where the physical processes take place. In view of this, our present elaboration, that belongs to the phenomenological realm, should be considered a step forward for setting up a viable tool for the actual investigation of strong-coupling regimes (first below the critical value), within a QFT framework where the dynamical ingredients are made transparent and under control.

Acknowledgements We gratefully thank Massimo Testa for very stimulating discussions, and $\mathrm{Si}$-xue Qin for illustrating in detail the issues related to the transverse vertex. C.M. acknowledges discussions with Jose Rodriguez-Quintero and the warm hospitality of INFN Sezione di Roma, where his INFN PostDoc fellowship for the NINPHA project has been granted.

Note added in proof During the production process we became aware of Ref. [113], devoted to the study of the quark propagator in Minkowski space by using a dispersive method.

Data Availability Statement This manuscript has no associated data or the data will not be deposited. [Authors' comment: This is a theoretical study and no experimental data has been listed.]

Open Access This article is licensed under a Creative Commons Attribution 4.0 International License, which permits use, sharing, adaptation, distribution and reproduction in any medium or format, as long as you give appropriate credit to the original author(s) and the source, provide a link to the Creative Commons licence, and indicate if changes were made. The images or other third party material in this article are included in the article's Creative Commons licence, unless indicated otherwise in a credit line to the material. If material is not included in the article's Creative Commons licence and your intended use is not permitted by statutory regulation or exceeds the permitted use, you will need to obtain permission directly from the copyright holder. To view a copy of this licence, visit http://creativecomm ons.org/licenses/by/4.0/.

Funded by $\mathrm{SCOAP}^{3}$.

\section{Appendix A: DSE for the fermion self-energy}

In this Appendix, the formal elaboration for obtaining the equation that determines the renormalized self-energy $\Sigma_{R}(\zeta ; p)$ is given in details.

The starting point is the integral equation fulfilled by the regularized self-energy $\Sigma(\zeta, \Lambda ; p)$ (see Itzykson and Zuber [89], p. 275, for the adopted notations, and also Fig. 8), that reads

$$
\begin{aligned}
\Sigma(\zeta, \Lambda ; p)= & i\left(-i e_{0}\right)^{2} \int_{\Lambda} \frac{d^{4} k}{(2 \pi)^{4}} \gamma^{\beta} S(\zeta, \Lambda ; k) \\
& \times \Gamma^{\alpha}(\zeta, \Lambda ; k, p) D_{\alpha \beta}(\zeta, \Lambda ; p-k)
\end{aligned}
$$

By introducing in Eq. (A.1) the following relation between regularized and renormalized quantities

$$
\begin{aligned}
& S(\zeta, \Lambda ; k)=Z_{2}(\zeta, \Lambda) S_{R}(\zeta, k) \\
& D_{\alpha \beta}(\zeta, \Lambda ; p-k)=Z_{3}(\zeta, \Lambda) D_{\alpha \beta}^{R}(\zeta ; p-k) \\
& \Gamma^{\alpha}(\zeta, \Lambda ; k, p)=\frac{\Gamma_{R}^{\alpha}(\zeta ; k, p)}{Z_{1}(\zeta, \Lambda)} \\
& e_{0}^{2} Z_{3}(\zeta, \Lambda)\left[\frac{Z_{2}(\zeta, \Lambda)}{Z_{1}(\zeta, \Lambda)}\right]^{2}=e_{R}^{2}
\end{aligned}
$$




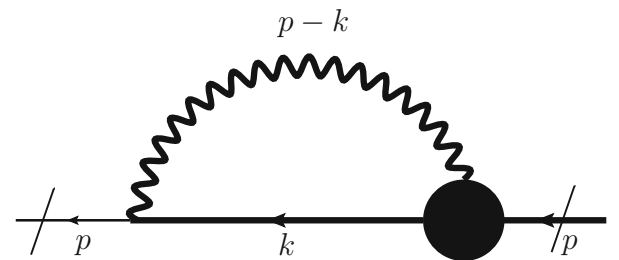

Fig. 8 The pictorial representation of the regularized fermion selfenergy in Eq. (A.3), with the external legs amputated. The thick lines are the renormalized propagators of both the fermion and the photon, respectively, while the thin one is the free fermion propagator The full dot represents the renormalized interaction vertex

one can rewrite

$\Sigma_{Z}(\zeta, \Lambda ; p)=-i Z_{1}(\zeta, \Lambda) e_{R}^{2} \int_{\Lambda} \frac{d^{4} k}{(2 \pi)^{4}} \gamma^{\beta} S_{R}(\zeta, k)$

$\Gamma_{R}^{\alpha}(\zeta ; k, p) D_{\alpha \beta}^{R}(\zeta ; p-k)$,

with $\Sigma_{Z}(\zeta, \Lambda ; p)=Z_{2} \Sigma(\zeta, \Lambda ; p)$. From Eq. (10), one gets the following integral equation for the renormalized selfenergy

$$
\begin{aligned}
& \Sigma_{R}(\zeta ; p)=p \mathcal{A}_{R}(\zeta ; p)+\mathcal{B}_{R}(\zeta ; p) \\
&= \Sigma_{Z}(\zeta, \Lambda ; p)-\left.\Sigma_{Z}(\zeta, \Lambda ; p)\right|_{p^{2}=\zeta^{2}} \\
&=-i Z_{1}(\zeta, \Lambda) e_{R}^{2} \int_{\Lambda} \frac{d^{4} k}{(2 \pi)^{4}} \gamma^{\beta} S_{R}(\zeta, k) \\
& \quad \times\left\{\Gamma_{R}^{\alpha}(\zeta ; k, p) D_{\alpha \beta}^{R}(\zeta ; p-k)\right. \\
&\left.\quad-\left[\Gamma_{R}^{\alpha}(\zeta ; k, p) D_{\alpha \beta}^{R}(\zeta ; p-k)\right]_{p^{2}=\zeta^{2}}\right\} .
\end{aligned}
$$

The scalar functions $\mathcal{A}_{R}(\zeta ; p)$ and $\mathcal{B}_{R}(\zeta ; p)$ can be obtained by evaluating the following traces

$$
\begin{aligned}
& \mathcal{A}_{R}(\zeta ; p)=\mathcal{T}_{A}(\zeta, \Lambda ; p)-\left.\mathcal{T}_{A}(\zeta, \Lambda ; p)\right|_{p^{2}=\zeta^{2}}, \\
& \mathcal{B}_{R}(\zeta ; p)=\mathcal{T}_{B}(\zeta, \Lambda ; p)-\left.\mathcal{T}_{B}(\zeta, \Lambda ; p)\right|_{p^{2}=\zeta^{2}}
\end{aligned}
$$

with

$$
\begin{aligned}
& \mathcal{T}_{A}(\zeta, \Lambda ; p)=\frac{1}{4 p^{2}} \operatorname{Tr}\left[p \Sigma_{Z}(\zeta, \Lambda ; p)\right], \\
& \mathcal{T}_{B}(\zeta, \Lambda ; p)=\frac{1}{4} \operatorname{Tr}\left[\Sigma_{Z}(\zeta, \Lambda ; p)\right] .
\end{aligned}
$$

Since in the Landau gauge the photon is transverse to the momentum transfer, only the transverse projection $T_{\beta \alpha} \Gamma_{R}^{\alpha}(\zeta ; k, p)$ is relevant (see Eq. (27) for the definition of $T_{\beta \alpha}$ ). It is important to notice that the transverse projection of the Ball-Chiu vertex is not vanishing, i.e. $T_{\beta \alpha} \Gamma_{R ; B C}^{\alpha}(\zeta ; k, p) \neq 0$. In particular, by using Eqs. (26) and (46) one gets

$$
\begin{aligned}
T_{\alpha}^{\beta} & \Gamma_{R ; B C}^{\alpha}(\zeta ; k, p)=\frac{\gamma_{T}^{\beta}}{2} F_{\mathcal{A}_{+}}(k, p, \zeta) \\
& -(p+\not k) p_{T}^{\beta} F_{\mathcal{A}_{-}}(k, p, \zeta)-2 p_{T}^{\beta} F_{\mathcal{B}}(k, p, \zeta),
\end{aligned}
$$

where the subscript $T$ on a four-vector means

$\mathcal{V}_{T}^{\beta}=\mathcal{V}^{\beta}-q^{\beta} \frac{\mathcal{V} \cdot q}{q^{2}}$,

with $q=p-k$ (notice that the photon is outcoming), so that $k_{T}^{\beta}=p_{T}^{\beta}$. Moreover, from Eq. (47) one has

$$
\begin{aligned}
& F_{\mathcal{A}_{+}}(k, p, \zeta)=2-\mathcal{A}_{R}(\zeta ; k)-\mathcal{A}_{R}(\zeta ; p), \\
& F_{\mathcal{A}_{-}}(k, p, \zeta)=\frac{\mathcal{A}_{R}(\zeta ; k)-\mathcal{A}_{R}(\zeta ; p)}{k^{2}-p^{2}} \\
&=-\int_{s_{t h}}^{\infty} d s \frac{\rho_{A}(s, \zeta)}{\left(k^{2}-s+i \epsilon\right)\left(p^{2}-s+i \epsilon\right)}, \\
& F_{\mathcal{B}}(k, p, \zeta)=\frac{\mathcal{B}_{R}(\zeta ; k)-\mathcal{B}_{R}(\zeta ; p)}{k^{2}-p^{2}} \\
&=-\int_{s_{t h}}^{\infty} d s \frac{\rho_{B}(s, \zeta)}{\left(k^{2}-s+i \epsilon\right)\left(p^{2}-s+i \epsilon\right)} .
\end{aligned}
$$

For the purely transverse component $\Gamma_{R ; T}^{\alpha}(\zeta ; k, p)$, Eq. (52), one has

$$
\begin{aligned}
T_{\alpha}^{\beta} & \Gamma_{R ; T}^{\alpha}(\zeta ; k, p)=-\frac{1}{2}\left[(p-k)^{2} \gamma_{T}^{\beta}\right. \\
& \left.+2 i \gamma_{5} \epsilon^{\beta \mu \nu \rho} \gamma_{\mu} p_{\nu} k_{\rho}\right] F_{\mathcal{A}_{-}}(k, p, \zeta),
\end{aligned}
$$

where $(p-k)_{T}^{\beta}=0$ has been used. Summing the two contributions to $\Gamma_{R ; T}^{\beta}$, one gets:

$$
\begin{aligned}
\bar{\Gamma}_{T}^{\beta} & =\frac{\gamma_{T}^{\beta}}{2} F_{\mathcal{A}_{+}}(k, p, \zeta)-\left[(p+\not k) p_{T}^{\beta}+(p-k)^{2} \frac{\gamma_{T}^{\beta}}{2}\right. \\
& \left.+i \gamma_{5} \epsilon^{\beta \alpha v \rho} \gamma_{\alpha} p_{v} k_{\rho}\right] F_{\mathcal{A}_{-}}(k, p, \zeta)-2 p_{T}^{\beta} F_{\mathcal{B}}(k, p, \zeta) .
\end{aligned}
$$

After inserting in Eq. (A.3), the expressions of the fermion and photon propagators in terms of the respective KL representations, i.e. Eqs. (15) and (39), and exploiting Eqs. (A.7) and (A.10), one can obtain the following expressions for the traces

$$
\begin{aligned}
& \mathcal{T}_{A(B)}(\zeta, \Lambda ; p)=-i Z_{1}(\zeta, \Lambda) e_{R}^{2} \int_{0}^{\infty} d \omega \bar{\sigma}_{\gamma}\left(\omega, \zeta, \zeta_{p}\right) \\
& \quad \times \int_{0}^{\infty} d s^{\prime} \int_{\Lambda} \frac{d^{4} k}{(2 \pi)^{4}} \frac{1}{(p-k)^{2}-\omega+i \epsilon} \frac{1}{k^{2}-s^{\prime}+i \epsilon} \\
& \quad \times \frac{1}{4} \operatorname{Tr}\left\{\left[k \bar{\sigma}_{V}\left(s^{\prime}, \zeta, s_{t h}^{\prime}\right)+\bar{\sigma}_{S}\left(s^{\prime}, \zeta, s_{t h}^{\prime}\right)\right] O_{A(B)}\right\},
\end{aligned}
$$

where

$O_{A}=\frac{1}{p^{2}} \bar{\Gamma}_{T}^{\beta} p \gamma_{\beta}, \quad O_{B}=\bar{\Gamma}_{T}^{\beta} \gamma_{\beta}$,

and

$$
\begin{aligned}
& \bar{\sigma}_{\gamma}\left(\omega, \zeta, \zeta_{p}\right)=\delta\left(\omega-\zeta_{p}^{2}\right)+\sigma_{\gamma}(\omega, \zeta) \Theta\left(\omega-\zeta_{p}^{2}\right), \\
& \bar{\sigma}_{S(V)}\left(s^{\prime}, \zeta, s_{t h}^{\prime}\right)=\delta\left(s^{\prime}-m^{2}(\zeta)\right)
\end{aligned}
$$




$$
+\sigma_{S(V)}\left(s^{\prime}, \zeta\right) \Theta\left(s^{\prime}-s_{t h}^{\prime}\right)
$$

\section{Appendix A.1: Traces evaluation}

From Eqs. (A.12) and (A.13), one gets the following traces. The one involved in the calculation of $\mathcal{T}_{A}$ is

$$
\begin{aligned}
& \frac{1}{4} \operatorname{Tr}\left\{\left[\not k \bar{\sigma}_{V}\left(s^{\prime}, \zeta, s_{t h}^{\prime}\right)+\bar{\sigma}_{S}\left(s^{\prime}, \zeta, s_{t h}^{\prime}\right)\right] O_{A}\right\} \\
& \quad=\bar{\sigma}_{V}\left(s^{\prime}, \zeta, s_{t h}^{\prime}\right) T r_{1}+\bar{\sigma}_{S}\left(s^{\prime}, \zeta, s_{t h}^{\prime}\right) T r_{2},
\end{aligned}
$$

where

$$
\begin{aligned}
\operatorname{Tr}_{1}= & -\frac{1}{p^{2}}\left\{\left[\frac{3}{2} k \cdot p-\frac{k^{2} p^{2}-(k \cdot p)^{2}}{(p-k)^{2}}\right] F_{\mathcal{A}_{+}}(k, p, \zeta)\right. \\
& +[\underbrace{\frac{k^{2} p^{2}-(k \cdot p)^{2}}{(p-k)^{2}}\left(k^{2}+p^{2}\right)}_{\Gamma_{B C}} \\
& -\underbrace{\left(\frac{3}{2} k \cdot p(p-k)^{2}-k^{2} p^{2}+(k \cdot p)^{2}\right)}_{\Gamma_{T_{3}}} \\
& -\underbrace{2\left(k^{2} p^{2}-(k \cdot p)^{2}\right)}_{\Gamma_{T_{8}}}] F_{\mathcal{A}_{-}}(k, p, \zeta)\},
\end{aligned}
$$

and

$\operatorname{Tr}_{2}=-\frac{2}{p^{2}} \frac{k^{2} p^{2}-(k \cdot p)^{2}}{(p-k)^{2}} F_{\mathcal{B}}(k, p, \zeta)$.

In Eq. (A.16), the underbraces emphasize the contributions generated by each term present in the vertex (see Eqs. (46) and (52)). This is motivated by the needed cooperation for eliminating the contribution produced by $\mathcal{A}_{R}(\zeta ; p)$ present in $F_{\mathcal{A}_{+}}$. Such a contribution generates a singular integral in $\mathcal{T}_{A}\left(\zeta, \Lambda ; p^{2}\right)$ that cannot be canceled by an analogous term in $\mathcal{T}_{A}\left(\zeta, \Lambda ; p^{2}=\zeta^{2}\right)$, since $\mathcal{A}_{R}(\zeta ; p=\zeta)=0$. Also in $F_{\mathcal{A}-}$ there is $\mathcal{A}_{R}(\zeta ; p)$, but in a combination with $\mathcal{A}_{R}(\zeta ; k)$, such that it does not plague the further calculation (see below). Notice that also in $\mathcal{T}_{B}\left(\zeta, \Lambda ; p^{2}\right)$ the same issue will be met. In conclusion, all the terms in the vertex function play an essential role for properly restoring the multiplicative renormalizability of the self-energy, as expressed in Eq. (A.4). This result is expected from the perturbative analysis (see, e.g., [42]), but it is gratifying to be achieved within a nonperturbative approach.

The aforementioned cancellation of $\mathcal{A}_{R}(\zeta ; p)$ in $\mathcal{T}_{A}$ can be attained by usefully recasting Eq. (A.16) as follows

$$
\begin{aligned}
\operatorname{Tr}_{1}= & -\frac{1}{p^{2}}\left\{\left[\frac{3}{2} k \cdot p-\frac{k^{2} p^{2}-(k \cdot p)^{2}}{(p-k)^{2}}\right]\right. \\
& \times\left[F_{\mathcal{A}_{+}}(k, p, \zeta)-\left(k^{2}-p^{2}\right) F_{\mathcal{A}_{-}}(k, p, \zeta)\right]
\end{aligned}
$$

$$
\begin{aligned}
& -\left[2 p^{2}\left(\frac{3}{2} k \cdot p-\frac{k^{2} p^{2}-(k \cdot p)^{2}}{(p-k)^{2}}\right)\right. \\
& \left.\left.+\left(k^{2} p^{2}-4(k \cdot p)^{2}\right)\right] F_{\mathcal{A}_{-}}(k, p, \zeta)\right\},
\end{aligned}
$$

Then, the problematic $\mathcal{A}_{R}(\zeta ; p)$ is canceled in the combination

$$
\begin{aligned}
& F_{\mathcal{A}_{+}}(k, p, \zeta)-\left(k^{2}-p^{2}\right) F_{\mathcal{A}_{-}}(k, p, \zeta) \\
& \quad=2\left[1-\mathcal{A}_{R}(\zeta ; k)\right] .
\end{aligned}
$$

obtained from the contributions produced by $T_{3}$ and $T_{8}$. It must be noticed in Eq. (A.18) that for getting the result one produces the term

$k^{2}-4(k \cdot p)^{2}$

that in principle can generate a singular integral. Indeed, an other fortunate cancellation takes place by exploiting the 4D angular integration and the difference between $\mathcal{T}_{A}\left(\zeta, \Lambda ; p^{2}\right)$ and $\mathcal{T}_{A}\left(\zeta, \Lambda ; \zeta^{2}\right)$ (see also Appendix A.4). Remarkably the factor of 4 is essential for obtaining the finite result.

For evaluating the trace in $\mathcal{T}_{B}$ (see Eq. (A.12)), one gets

$$
\begin{aligned}
& \operatorname{Tr}\left\{\left[k \bar{\sigma}_{V}\left(s^{\prime}, \zeta, s_{t h}^{\prime}\right)+\bar{\sigma}_{S}\left(s^{\prime}, \zeta, s_{t h}^{\prime}\right)\right] O_{B}\right\} \\
& \quad=\bar{\sigma}_{V}\left(s^{\prime}, \zeta, s_{t h}^{\prime}\right) T r_{3}+\bar{\sigma}_{S}\left(s^{\prime}, \zeta, s_{t h}^{\prime}\right) T r_{4}
\end{aligned}
$$

where

$\operatorname{Tr}_{3}=-2 \frac{k^{2} p^{2}-(k \cdot p)^{2}}{(p-k)^{2}} F_{\mathcal{B}}(k, p, \zeta)$,

and

$$
\begin{aligned}
T r_{4} & =\frac{3}{2}\left[F_{\mathcal{A}_{+}}(k, p, \zeta)-\left(k^{2}-p^{2}\right) F_{\mathcal{A}_{-}}(k, p, \zeta)\right] \\
& -\left[2 \frac{k^{2} p^{2}-(k \cdot p)^{2}}{(p-k)^{2}}+3\left(p^{2}-k \cdot p\right)\right] F_{\mathcal{A}_{-}}(k, p, \zeta) .
\end{aligned}
$$

Finally, collecting all the results, one has the following expressions for $\mathcal{T}_{A}(\zeta, \Lambda ; p)$ and $\mathcal{T}_{B}(\zeta, \Lambda ; p)$

$$
\begin{aligned}
\mathcal{T}_{A}(\zeta, \Lambda ; p)=i Z_{1}(\zeta, \Lambda) e_{R}^{2} \frac{3}{p^{2}} \int_{0}^{\infty} d \omega \bar{\sigma}_{\gamma}\left(\omega, \zeta, \zeta_{p}\right) \\
\quad \times \int_{0}^{\infty} d s^{\prime} \int_{\Lambda} \frac{d^{4} k}{(2 \pi)^{4}} \frac{1}{(p-k)^{2}-\omega+i \epsilon} \frac{1}{k^{2}-s^{\prime}+i \epsilon} \\
\quad \times\left\{\overline { \sigma } _ { V } ( s ^ { \prime } , \zeta , s _ { t h } ^ { \prime } ) \left[\left(k \cdot p-\frac{2}{3} \mathcal{R}(k, p)\right)\right.\right. \\
\quad \times\left(1-\mathcal{A}_{R}(\zeta ; k)\right)-\left(p^{2}\left(k \cdot p-\frac{2}{3} \mathcal{R}(k, p)\right)\right. \\
\left.\left.\quad+\frac{k^{2} p^{2}-4(k \cdot p)^{2}}{3}\right) F_{\mathcal{A}}(k, p, \zeta)\right] \\
\left.+\frac{2}{3} \bar{\sigma}_{S}\left(s^{\prime}, \zeta, s_{t h}^{\prime}\right) \mathcal{R}(k, p) F_{\mathcal{B}}(k, p, \zeta)\right\},
\end{aligned}
$$


and

$$
\begin{aligned}
& \mathcal{T}_{B}(\zeta, \Lambda ; p)=-i Z_{1}(\zeta, \Lambda) e_{R}^{2} 3 \int_{0}^{\infty} d \omega \bar{\sigma}_{\gamma}\left(\omega, \zeta, \zeta_{p}\right) \\
& \quad \times \int_{0}^{\infty} d s^{\prime} \int_{\Lambda} \frac{d^{4} k}{(2 \pi)^{4}} \frac{1}{(p-k)^{2}-\omega+i \epsilon} \frac{1}{k^{2}-s^{\prime}+i \epsilon} \\
& \quad \times\left\{\overline { \sigma } _ { S } ( s ^ { \prime } , \zeta , s _ { t h } ^ { \prime } ) \left[1-\mathcal{A}_{R}(\zeta ; k)\right.\right. \\
& \left.\quad-\left(p^{2}-k \cdot p+\frac{2}{3} \mathcal{R}(k, p)\right) F_{\mathcal{A}_{-}}(k, p, \zeta)\right] \\
& \left.-\bar{\sigma}_{V}\left(s^{\prime}, \zeta, s_{t h}^{\prime}\right) \frac{2}{3} \mathcal{R}(k, p) F_{\mathcal{B}}(k, p, \zeta)\right\},
\end{aligned}
$$

with

$\mathcal{R}(k, p)=\frac{k^{2} p^{2}-(k \cdot p)^{2}}{(p-k)^{2}}=\frac{q^{2} p^{2}-(q \cdot p)^{2}}{q^{2}}$.

with $q=p-k$.

By using Eq. (13) for $\mathcal{A}_{R}(\zeta ; k)$ and (47) for $F_{\mathcal{A}_{-}}$and $F_{\mathcal{B}}$, one can write $\mathcal{T}_{A}$ and $\mathcal{T}_{B}$, as follows

$$
\begin{aligned}
& \mathcal{T}_{A}(\zeta, \Lambda ; p)=i Z_{1}(\zeta, \Lambda) e_{R}^{2} \frac{3}{p^{2}} \int_{0}^{\infty} d \omega \bar{\sigma}_{\gamma}\left(\omega, \zeta, \zeta_{p}\right) \\
& \quad \times\left\{\int_{0}^{\infty} d s^{\prime} \bar{\sigma}_{V}\left(s^{\prime}, \zeta, s_{t h}^{\prime}\right) \mathcal{I}_{1}\left(p, \omega, s^{\prime}\right)\right. \\
& \quad+\int_{s_{t h}}^{\infty} d s \rho_{A}(s, \zeta) \int_{0}^{\infty} d s^{\prime} \bar{\sigma}_{V}\left(s^{\prime}, \zeta, s_{t h}^{\prime}\right) \\
& \times\left[\frac{\mathcal{I}_{1}\left(p, \omega, s^{\prime}\right)}{\left(\zeta^{2}-s+i \epsilon\right)}-\mathcal{I}_{4}\left(p, \omega, s^{\prime}, s\right)\right] \\
& +\int_{s_{t h}}^{\infty} d s \frac{\rho_{A}(s, \zeta)}{\left(p^{2}-s+i \epsilon\right)} \int_{s_{t h}}^{\infty} d s^{\prime} \bar{\sigma}_{V}\left(s^{\prime}, \zeta, s_{t h}^{\prime}\right) \\
& \quad \times\left[p^{2} \mathcal{I}_{4}\left(p, \omega, s^{\prime}, s\right)+\frac{1}{3} \mathcal{I}_{5}\left(p, \omega, s^{\prime}, s\right)\right]-\frac{2}{3} \int_{s_{t h}}^{\infty} d s \\
& \left.\quad \times \frac{\rho_{B}(s, \zeta)}{\left(p^{2}-s+i \epsilon\right)} \int_{s_{t h}}^{\infty} d s^{\prime} \bar{\sigma}_{S}\left(s^{\prime}, \zeta, s_{t h}^{\prime}\right) \mathcal{I}_{3}\left(p, \omega, s^{\prime}, s\right)\right\}
\end{aligned}
$$

and

$$
\begin{aligned}
& \mathcal{T}_{B}(\zeta, \Lambda ; p)=-i 3 Z_{1}(\zeta, \Lambda) e_{R}^{2} \int_{0}^{\infty} d \omega \bar{\sigma}_{\gamma}\left(\omega, \zeta, \zeta_{p}\right) \\
& \quad \times\left\{\int_{0}^{\infty} d s^{\prime} \bar{\sigma}_{S}\left(s^{\prime}, \zeta, s_{t h}^{\prime}\right) \mathcal{I}_{0}\left(p, \omega, s^{\prime}\right)\right. \\
& \quad+\int_{s_{t h}}^{\infty} d s \rho_{A}(s, \zeta) \int_{0}^{\infty} d s^{\prime} \bar{\sigma}_{S}\left(s^{\prime}, \zeta, s_{t h}^{\prime}\right) \\
& \quad \times\left[\frac{\mathcal{I}_{0}\left(p, \omega, s^{\prime}\right)}{\left(\zeta^{2}-s+i \epsilon\right)}-\mathcal{I}_{2}\left(p, \omega, s^{\prime}, s\right)\right] \\
& \quad+\int_{s_{t h}}^{\infty} d s \frac{\rho_{A}(s, \zeta)}{\left(p^{2}-s+i \epsilon\right)} \int_{0}^{\infty} d s^{\prime} \bar{\sigma}_{S}\left(s^{\prime}, \zeta, s_{t h}^{\prime}\right) \\
& \quad \times\left(p^{2} \mathcal{I}_{2}\left(p, \omega, s^{\prime}, s\right)-\mathcal{I}_{4}\left(p, \omega, s^{\prime}, s\right)\right)
\end{aligned}
$$

$$
\begin{aligned}
& +\frac{2}{3} \int_{s_{t h}}^{\infty} d s \frac{\rho_{B}(s, \zeta)}{\left(p^{2}-s+i \epsilon\right)} \\
& \left.\times \int_{0}^{\infty} d s^{\prime} \bar{\sigma}_{V}\left(s^{\prime}, \zeta, s_{t h}^{\prime}\right) \mathcal{I}_{3}\left(p, \omega, s^{\prime}, s\right)\right\}
\end{aligned}
$$

where

$$
\begin{aligned}
\mathcal{I}_{0}\left(p, \omega, s^{\prime}\right)= & \int_{\Lambda} \frac{d^{4} k}{(2 \pi)^{4}} \frac{1}{(p-k)^{2}-\omega+i \epsilon} \\
& \times \frac{1}{k^{2}-s^{\prime}+i \epsilon} \\
\mathcal{I}_{1}\left(p, \omega, s^{\prime}\right)= & \int_{\Lambda} \frac{d^{4} k}{(2 \pi)^{4}}\left[k \cdot p-\frac{2}{3} \mathcal{R}(k, p)\right] \\
& \times \frac{1}{(p-k)^{2}-\omega+i \epsilon} \frac{1}{k^{2}-s^{\prime}+i \epsilon} \\
\mathcal{I}_{2}\left(p, \omega, s^{\prime}, s\right)= & \int_{\Lambda} \frac{d^{4} k}{(2 \pi)^{4}} \frac{1}{(p-k)^{2}-\omega+i \epsilon} \\
& \times \frac{1}{k^{2}-s^{\prime}+i \epsilon} \frac{1}{k^{2}-s+i \epsilon} \\
& \times \frac{1}{k^{2}-s^{\prime}+i \epsilon} \frac{1}{k^{2}-s+i \epsilon} \\
\mathcal{I}_{3}\left(p, \omega, s^{\prime}, s\right)= & \int_{\Lambda} \frac{d^{4} k}{(2 \pi)^{4}} \mathcal{R}(k, p) \frac{1}{(p-k)^{2}-\omega+i \epsilon} \\
\mathcal{I}_{4}\left(p, \omega, s^{\prime}, s\right)= & \int_{\Lambda} \frac{d^{4} k}{(2 \pi)^{4}}\left[k \cdot p-\frac{2}{3} \mathcal{R}(k, p)\right] \\
& \times \frac{1}{(p-k)^{2}-\omega+i \epsilon} \frac{1}{k^{2}-s^{\prime}+i \epsilon} \frac{1}{k^{2}-s+i \epsilon} \\
\mathcal{I}_{5}\left(p, \omega, s^{\prime}, s\right)= & \int_{\Lambda} \frac{d^{4} k}{(2 \pi)^{4}} \frac{k^{2} p^{2}-4(k \cdot p)^{2}}{(p-k)^{2}-\omega+i \epsilon} \\
& \times \frac{1}{k^{2}-s^{\prime}+i \epsilon} \frac{1}{k^{2}-s+i \epsilon}
\end{aligned}
$$

with $\omega, s^{\prime}, s \geq 0$.

It has to point out that $\mathcal{I}_{0}, \mathcal{I}_{1}$, and $\mathcal{I}_{5}$ are divergent integrals for $d=4$, and only after applying i) the dimensional regularization and ii) the subtraction of the corresponding integrals evaluated at $p^{2}=\zeta^{2}$, one gets finite results for $\mathcal{A}_{R}$ and $\mathcal{B}_{R}$, as it will be shown in Appendix A.3 and Appendix A.4, respectively.

Differently, the three integrals $\mathcal{I}_{2}, \mathcal{I}_{3}$ and $\mathcal{I}_{4}$ are finite, and they can be evaluated by (i) applying the Feynman parametric formula and introducing a new variable $q=k-\alpha p$ ( $\alpha$ is a proper combination of Feynman parameters); (ii) changing the variable $q_{0} \rightarrow i q_{4}$ and iii) eventually using $4 \mathrm{D}$ polar coordinates, $q_{E} \equiv\left\{q_{x}, q_{y}, q_{z}, q_{4}\right\}$, given by

$q_{E} \equiv \rho\left\{\sin \theta_{2} \sin \theta_{1} \cos \phi, \sin \theta_{2} \sin \theta_{1} \sin \phi\right.$, $\left.\sin \theta_{2} \cos \theta_{1}, \cos \theta_{2}\right\}$. 
Appendix A.2: Analytic integrals

The evaluation of the analytic integrals in $\mathcal{T}_{A}(\zeta, \Lambda ; p)$ and $\mathcal{T}_{B}(\zeta, \Lambda ; p)$ represents the most lengthy part of the formal elaboration. It is helpful to recall that our goal is to achieve a form of both $\mathcal{A}_{R}$ and $\mathcal{B}_{R}$ suitable for exploiting the uniqueness of the NWFs $\rho_{A}$ and $\rho_{B}$, as suggested by a theorem demonstrated by Nakanishi for a generic n-leg transition amplitude [57].

To proceed in a simple way, it is very useful to consider spacelike values for the external momentum $p$. This choice, as it becomes immediately clear, simplifies a lot the formal elaboration, and it is not restrictive, since the NWFs do not depend upon the values of the external momentum, but noteworthy they are used for obtaining the scalar functions $\mathcal{A}_{R}$ and $\mathcal{B}_{R}$ at any value of $p^{2}$.

For the finite integral $\mathcal{I}_{2}$ one gets

$$
\begin{aligned}
\mathcal{I}_{2}\left(p, \omega, s^{\prime}, s\right)=\int_{0}^{1} d \xi \int_{0}^{1-\xi} d v \int_{2} \frac{d^{4} q}{(2 \pi)^{4}} \\
\times \frac{\left[q^{2}+\xi(1-\xi) p^{2}-\xi \omega-v s^{\prime}-(1-\xi-v) s+i \epsilon\right]^{3}}{\left[\begin{array}{l}
1 \\
=
\end{array}\right.} \\
\quad \times \frac{1}{(4 \pi)^{2}} \int_{0}^{1} d \xi \int_{0}^{1-\xi} d v
\end{aligned}
$$

The last step can be easily carried out without any concern, given the aforementioned choice of $p^{2}<0$.

The second finite integral, $\mathcal{I}_{3}$, becomes

$$
\begin{aligned}
\mathcal{I}_{3}\left(p, \omega, s^{\prime}, s\right)=\int_{0}^{1} d \xi \int_{0}^{1-\xi} d v \int_{0}^{1-\xi-v} d w \int \frac{d^{4} q}{(2 \pi)^{4}} \\
\times \frac{6\left[q^{2} p^{2}-(q \cdot p)^{2}\right]}{\left[q^{2}+\mathcal{A}_{4}(v, w) p^{2}-\xi \omega-v s^{\prime}-w s+i \epsilon\right]^{4}} \\
=\frac{3}{2} p^{2} \frac{i}{(4 \pi)^{2}} \int_{0}^{1} d \xi \int_{0}^{1-\xi} d v \int_{0}^{1-\xi-v} d w \\
\quad \times \frac{1}{\left[\mathcal{A}_{4}(v, w) p^{2}-\xi \omega-v s^{\prime}-w s+i \epsilon\right]}
\end{aligned}
$$

with $\mathcal{A}_{4}(v, w)=(v+w)(1-v-w)$.

Finally, $\mathcal{I}_{4}$ can be evaluated by using the result in Eq. (A.36), i.e.

$$
\begin{aligned}
\mathcal{I}_{4}\left(p, \omega, s^{\prime}, s\right)=2 p^{2} \int_{0}^{1} d \xi \int_{0}^{1-\xi} d v \int \frac{d^{4} q}{(2 \pi)^{4}} \\
\times \frac{\xi}{\left[q^{2}+\xi(1-\xi) p^{2}-\xi \omega-v s^{\prime}-(1-\xi-v) s+i \epsilon\right]^{3}}
\end{aligned}
$$

$$
\begin{aligned}
& -\frac{2}{3} \mathcal{I}_{3}\left(p, \omega, s^{\prime}, s\right) \\
= & p^{2} \frac{i}{(4 \pi)^{2}} \int_{0}^{1} d \xi \int_{0}^{1-\xi} d v \\
& \times\left\{\frac{\xi}{\left[\xi(1-\xi) p^{2}-\xi \omega-v s^{\prime}-(1-\xi-v) s+i \epsilon\right]}\right. \\
& \left.-\int_{0}^{1-\xi-v} d w \frac{1}{\left[\mathcal{A}_{4}(v, w) p^{2}-\xi \omega-v s^{\prime}-w s+i \epsilon\right]}\right]
\end{aligned}
$$

In the above 4D integration on $q$ the subscript $\Lambda$ has been removed since the regularization is not necessary.

Appendix A.3: The $\mathcal{B}_{R}$ contribution to the fermion self-energy

Let us start the evaluation of the contribution $\mathcal{B}_{R}$, since it contains only one divergent integral, i.e. $\mathcal{I}_{0}$, Eq. (A.28). To get a finite value for the contribution from $\mathcal{I}_{0}$, it is compulsory to exploit both the dimensional regularization, that legitimates the variable shift in the integrand, and the subtraction of the corresponding term in $\left.\mathcal{T}_{B}(\zeta, \Lambda ; p)\right|_{p^{2}=\zeta^{2}}$, as shown in Eq. (A.5). With this in mind, we simplify the formal elaboration removing the dependence upon $\Lambda$ in what follows, and directly using the $4 \mathrm{D}$ integration. $\mathcal{B}_{R}(\zeta ; p)$ in Eq. (A.5) (see also Eq. (A.27)) reads

$$
\begin{aligned}
& \frac{\mathcal{B}_{R}(\zeta ; p)}{\left(\zeta^{2}-p^{2}\right)}=3 Z_{1}(\zeta) e_{R}^{2} \int_{0}^{\infty} d \omega \bar{\sigma}_{\gamma}\left(\omega, \zeta, \zeta_{p}\right) \int_{0}^{\infty} d s^{\prime} \\
& \quad \times\left\{\bar{\sigma}_{S}\left(s^{\prime}, \zeta, s_{t h}^{\prime}\right) \mathcal{D}_{0}\left(p, \zeta, \omega, s^{\prime}\right)+\int_{s_{t h}}^{\infty} d s \rho_{A}(s, \zeta)\right. \\
& \quad \times \bar{\sigma}_{S}\left(s^{\prime}, \zeta, s_{t h}^{\prime}\right)\left[\frac{\mathcal{D}_{0}\left(p, \zeta, \omega, s^{\prime}\right)}{\zeta^{2}-s+i \epsilon}-\mathcal{D}_{2}\left(p, \zeta, \omega, s^{\prime}, s\right)\right. \\
& \left.\quad+\mathcal{D}_{24}\left(p, \zeta, \omega, s^{\prime}, s\right)\right]+\int_{s_{t h}}^{\infty} d s \rho_{B}(s, \zeta)^{\prime} \\
& \left.\quad \times \bar{\sigma}_{V}\left(s^{\prime}, \zeta, s_{t h}^{\prime}\right) \mathcal{D}_{3}\left(p, \zeta, \omega, s^{\prime}, s\right)\right\}
\end{aligned}
$$

where $Z_{1}(\zeta)=Z_{1}(\zeta, \Lambda \rightarrow \infty)$ and the differences of integrals (see Eqs. (A.28), (A.35), (A.36) and (A.37)) are defined as follows

$$
\mathcal{D}_{0}\left(p, \zeta, \omega, s^{\prime}\right)=-i \frac{\left[\mathcal{I}_{0}\left(p, \omega, s^{\prime}\right)-\mathcal{I}_{0}\left(\zeta, \omega, s^{\prime}\right)\right]}{\left(\zeta^{2}-p^{2}\right)}
$$

$\mathcal{D}_{2}\left(p, \zeta, \omega, s^{\prime}, s\right)=-i \frac{\left[\mathcal{I}_{2}\left(p, \omega, s^{\prime}, s\right)-\mathcal{I}_{2}\left(\zeta, \omega, s^{\prime}, s\right)\right]}{\left(\zeta^{2}-p^{2}\right)}$

$\mathcal{D}_{3}\left(p, \zeta, \omega, s^{\prime}, s\right)=-i \frac{2}{3} \frac{1}{\left(\zeta^{2}-p^{2}\right)}$ 
$\times\left[\frac{\mathcal{I}_{3}\left(p, \omega, s^{\prime}, s\right)}{p^{2}-s+i \epsilon}-\frac{\mathcal{I}_{3}\left(\zeta, \omega, s^{\prime}, s\right)}{\zeta^{2}-s+i \epsilon}\right]$

(A.41)

$$
\begin{aligned}
\mathcal{D}_{24}\left(p, \zeta, \omega, s^{\prime}, s\right)= & -i \frac{1}{\left(\zeta^{2}-p^{2}\right)} \\
& \times\left[\frac{p^{2} \mathcal{I}_{2}\left(p, \omega, s^{\prime}, s\right)-\mathcal{I}_{4}\left(p, \omega, s^{\prime}, s\right)}{p^{2}-s+i \epsilon}\right. \\
& \left.-\frac{\zeta^{2} \mathcal{I}_{2}\left(\zeta, \omega, s^{\prime}, s\right)-\mathcal{I}_{4}\left(\zeta, \omega, s^{\prime}, s\right)}{\zeta^{2}-s+i \epsilon}\right]
\end{aligned}
$$

The actual evaluation of the differences is briefly sketched. The first one, $\mathcal{D}_{0}$, can be obtained from Eq. (A.28) and recalling the need of the regularization, viz

$$
\begin{gathered}
\mathcal{D}_{0}\left(p, \zeta, \omega, s^{\prime}\right)=\frac{-i}{\left(\zeta^{2}-p^{2}\right)} \int_{0}^{1} d \xi \int \frac{d^{4} q}{(2 \pi)^{4}} \\
\times \frac{\times\left[\chi_{0}(p, q)-. \chi_{0}(\zeta, q)\right]=\frac{1}{(4 \pi)^{2}} \int_{0}^{1} d \xi \int_{0}^{1} d v}{\left\{\xi(1-\xi)\left[v \zeta^{2}+(1-v) p^{2}\right]-\xi \omega-(1-\xi) s^{\prime}+i \epsilon\right\}} \\
=\frac{1}{(4 \pi)^{2}} \int_{0}^{1} d v \int_{0}^{\infty} d y \int_{0}^{1} d \xi \delta\left[y-\frac{\xi \omega+(1-\xi) s^{\prime}}{\xi(1-\xi)}\right] \\
\times \frac{1}{\left[v \zeta^{2}+(1-v) p^{2}-y+i \epsilon\right]}=-\frac{1}{(4 \pi)^{2}} \int_{0}^{1} d v \int_{0}^{\infty} d y \\
\quad \times \int_{0}^{1} d \xi \frac{\Theta\left[\xi(1-\xi) y-\xi \omega-(1-\xi) s^{\prime}\right]}{\left[v \zeta^{2}+(1-v) p^{2}-y+i \epsilon\right]^{2}},
\end{gathered}
$$

where

$$
\chi_{0}(p, q)=\frac{1}{\left[q^{2}+\xi(1-\xi) p^{2}-\xi \omega-(1-\xi) s^{\prime}+i \epsilon\right]^{2}}
$$

and the formal manipulations are allowed by the dimensional regularization. Eventually, the last line has been introduced after applying an integration by parts for preparing the application of the uniqueness theorem [57] to the NWF $\rho_{B}$. Notice that $\xi \omega+(1-\xi) s^{\prime}+\eta \geq 0$ and the exchange of the integration on $\eta$ and on $y$ has been assumed to be allowed.

From Eq. (A.35), one gets

$$
\begin{gathered}
\mathcal{D}_{2}\left(p, \zeta, \omega, s^{\prime}, s\right)=\frac{1}{(4 \pi)^{2}} \int_{0}^{1} d v \int_{0}^{\infty} d y \int_{0}^{1} \frac{d \xi}{\xi(1-\xi)} \\
\times \int_{0}^{1-\xi} d z \frac{\delta\left[y-\frac{\xi \omega+z s^{\prime}+(1-\xi-z) s}{\xi(1-\xi)}\right]}{\left[v \zeta^{2}+(1-v) p^{2}-y+i \epsilon\right]^{2}}
\end{gathered}
$$

To evaluate $\mathcal{D}_{3}$, one can introduce the following difference with $A>0$ and $B>0$

$$
\begin{aligned}
\mathcal{D}= & \frac{p^{2}}{\left(p^{2}-s+i \epsilon\right)\left(A p^{2}-B+i \epsilon\right)} \\
& -\frac{\zeta^{2}}{\left(\zeta^{2}-s+i \epsilon\right)\left(A \zeta^{2}-B+i \epsilon\right)} \\
= & \frac{1}{(s A-B)}\left[\frac{s}{\left(p^{2}-s+i \epsilon\right)}-\frac{B / A}{\left(p^{2}-B / A+i \epsilon\right)}\right. \\
& \left.-\frac{s}{\left(\zeta^{2}-s+i \epsilon\right)}+\frac{B / A}{\left(\zeta^{2}-B / A+i \epsilon\right)}\right] \\
= & -\frac{s}{[s A-B)} \int_{0}^{1} d v\left\{\frac{p^{2}}{\left[v \zeta^{2}+(1-v) p^{2}-s+i \epsilon\right]^{2}}\right. \\
= & -\left(\zeta^{2}-p^{2}\right) \int_{0}^{1} d v \int_{0}^{\infty} d y y \\
& \times \frac{\Delta^{\prime}[y-s+(s-B / A)]}{\left[v \zeta^{2}+(1-v) p^{2}-y+i \epsilon\right]^{2}}, \quad(\mathrm{~A} .46)
\end{aligned}
$$

where $\Delta^{\prime}$ indicates

$$
\begin{aligned}
\Delta^{\prime} & {[y-s+(s A-B) / A] } \\
& =\frac{\delta[y-s+(s A-B) / A]-\delta(y-s)}{(s A-B)}
\end{aligned}
$$

From (A.36) and by suitably modifying Eq. (A.46), one has

$$
\begin{aligned}
& \mathcal{D}_{3}\left(p, \zeta, \omega, s^{\prime}, s\right)=-\frac{1}{(4 \pi)^{2}} \int_{0}^{1} d v \int_{0}^{\infty} d y \\
& \quad \times \frac{1}{\left[\left(v \zeta^{2}+(1-v) p^{2}\right)-y+i \epsilon\right]^{2}} \int_{0}^{1} d \xi \\
& \quad \times \int_{0}^{1-\xi} d t \int_{0}^{1-\xi-t} d w y \\
& \quad \times \Delta^{\prime}\left[y-s+\left(s \mathcal{A}_{4}(t, w)-\xi \omega-t s^{\prime}-w s\right) / \mathcal{A}_{4}(t, w)\right],
\end{aligned}
$$

with $\mathcal{A}_{4}(t, w)=(t+w)(1-t-w)$. Finally, from Eqs. (A.35), (A.37), (A.46) and (A.48), one writes

$$
\begin{aligned}
& \mathcal{D}_{24}\left(p, \zeta, \omega, s^{\prime}, s\right)=\frac{1}{(4 \pi)^{2}\left(\zeta^{2}-p^{2}\right)} \int_{0}^{1} d \xi(1-\xi) \\
& \times \int_{0}^{1-\xi} d t\left\{\frac{p^{2}}{\left(p^{2}-s+i \epsilon\right)}\right.
\end{aligned}
$$


34 Page 22 of 35

Eur. Phys. J. C (2021) 81:34

$$
\begin{aligned}
& \times \frac{1}{\left[\xi(1-\xi) p^{2}-\xi \omega-t s^{\prime}-(1-\xi-t) s+i \epsilon\right]} \\
& -\frac{\zeta^{2}}{\left(\zeta^{2}-s+i \epsilon\right)} \\
& \left.\times \frac{1}{\left[\xi(1-\xi) \zeta^{2}-\xi \omega-t s^{\prime}-(1-\xi-t) s+i \epsilon\right]}\right\} \\
& +\mathcal{D}_{3}\left(p, \zeta, \omega, s, s^{\prime}\right) \\
& =\frac{1}{(4 \pi)^{2}} \int_{0}^{1} d v \int_{0}^{\infty} d y y \int_{0}^{1} d \xi \int_{0}^{1-\xi} d t \\
& \times\left\{(1-\xi) \frac{\Delta^{\prime}\left[y-s+\frac{\left(\xi(1-\xi) s-\xi \omega-t s^{\prime}-(1-\xi-t) s\right.}{\xi(1-\xi)}\right]}{\left[\left(v \zeta^{2}+(1-v) p^{2}\right)-y+i \epsilon\right]^{2}}\right. \\
& \left.+\int_{0}^{1-\xi-t} d w \frac{\Delta^{\prime}\left[y-s+\frac{\left(\mathcal{A}_{4}(t, w) s-\xi \omega-t s^{\prime}-w s\right)}{\mathcal{A}_{4}(t, w)}\right]}{\left[\left(v \zeta^{2}+(1-v) p^{2}\right)-y+i \epsilon\right]}\right\} .
\end{aligned}
$$

Inserting Eqs. (A.43), (A.45), (A.48), and (A.49), one gets

$$
\begin{aligned}
& \frac{\mathcal{B}_{R}(\zeta ; p)}{\left(\zeta^{2}-p^{2}\right)}=\frac{-3}{(4 \pi)^{2}} Z_{1}(\zeta) e_{R}^{2} \int_{0}^{1} d v \int_{0}^{\infty} d y \int_{0}^{\infty} d \omega \\
& \quad \times \int_{0}^{1} d \xi \frac{\bar{\sigma}_{\gamma}\left(\omega, \zeta, \zeta_{p}\right)}{\left[\left(v \zeta^{2}+(1-v) p^{2}\right)-y+i \epsilon\right]^{2}} \\
& \quad \times \int_{0}^{\infty} d s^{\prime}\left\{\overline { \sigma } _ { S } ( s ^ { \prime } , \zeta , s _ { t h } ^ { \prime } ) \left[\Theta\left[(1-\xi)\left(y \xi-s^{\prime}\right)-\xi \omega\right]\right.\right. \\
& \quad+\int_{s_{t h}}^{\infty} d s \rho_{A}(s, \zeta)\left(\mathcal{C}_{A S}^{(0)}\left(\zeta, \omega, s, s^{\prime}, \xi, y\right)\right. \\
& \left.\left.\quad+y \mathcal{C}_{A S}^{(1)}\left(\zeta, \omega, s, s^{\prime}, \xi, y\right)\right)\right] \\
& \quad+y \bar{\sigma}_{V}\left(s^{\prime}, \zeta, s_{t h}^{\prime}\right) \int_{s_{t h}}^{\infty} d s \rho_{B}(s, \zeta) \int_{0}^{1-\xi} d t \int_{0}^{1-\xi-t} d w \\
& \left.\quad \times \Delta^{\prime}\left[y-s+\frac{\mathcal{A}_{4}(t, w) s-\xi \omega-t s^{\prime}-w s}{\mathcal{A}_{4}(t, w)}\right]\right\}, \quad(\mathrm{A} .50)
\end{aligned}
$$

with $Z_{1}(\zeta)=Z_{1}(\zeta, \Lambda \rightarrow \infty)$,

$$
\begin{gathered}
\mathcal{C}_{A S}^{(0)}\left(\zeta, \omega, s, s^{\prime}, \xi, y\right)=\frac{\Theta\left[y \xi(1-\xi)-\xi \omega-(1-\xi) s^{\prime}\right]}{\zeta^{2}-s+i \epsilon} \\
+\frac{1}{\xi(1-\xi)} \int_{0}^{1-\xi} d z \delta\left[y-\frac{\xi \omega+z s^{\prime}+(1-\xi-z) s}{\xi(1-\xi)}\right],
\end{gathered}
$$

and

$$
\begin{aligned}
& \mathcal{C}_{A S}^{(1)}\left(\zeta, \omega, s, s^{\prime}, \xi, y\right)=\int_{0}^{1-\xi} d t\{(1-\xi) \\
& \quad \times \Delta^{\prime}\left[y-s+\frac{\xi(1-\xi) s-\xi \omega-t s^{\prime}-(1-\xi-t) s}{\xi(1-\xi)}\right]
\end{aligned}
$$

$$
\left.+\int_{0}^{1-\xi-t} d w \Delta^{\prime}\left[y-s+\frac{\mathcal{A}_{4}(t, w) s-\xi \omega-t s^{\prime}-w s}{\mathcal{A}_{4}(t, w)}\right]\right\} .
$$

Appendix A.4: The $\mathcal{A}_{R}$ contribution

In order to evaluate $\mathcal{A}_{R}$, (recall $p^{2}<0$, but without loss of generality on the final result for the NWFs) one has to face with the divergent behavior of $\mathcal{I}_{1}$, Eq. (A.29), and $\mathcal{I}_{5}$, Eq. (A.33). The strategy is exactly the same we have applied to $\mathcal{I}_{0}$ in the subsec. Appendix A.3, combining the dimensional regularization for shifting the integration variable and then exploiting the subtraction.

One can write from Eq. (A.5)

$$
\begin{aligned}
& \frac{\mathcal{A}_{R}(\zeta ; p)}{\zeta^{2}-p^{2}}=-3 Z_{1}(\zeta, \Lambda \rightarrow \infty) e_{R}^{2} \int_{0}^{\infty} d \omega \bar{\sigma}_{\gamma}\left(\omega, \zeta, \zeta_{p}\right) \\
& \quad \times \int_{0}^{\infty} d s^{\prime}\left\{\bar{\sigma}_{V}\left(s^{\prime}, \zeta, s_{t h}^{\prime}\right) \mathcal{D}_{1}\left(p, \zeta, \omega, s^{\prime}\right)\right. \\
& \quad+\int_{s_{t h}}^{\infty} d s \rho_{A}(s, \zeta) \bar{\sigma}_{V}\left(s^{\prime}, \zeta, s_{t h}^{\prime}\right)\left[\frac{\mathcal{D}_{1}\left(p, \zeta, \omega, s^{\prime}\right)}{\left(\zeta^{2}-s+i \epsilon\right)}\right. \\
& \quad-\mathcal{D}_{4}\left(p, \zeta, \omega, s^{\prime}, s\right) \\
& \left.\quad+\mathcal{D}_{4}^{\prime}\left(p, \zeta, \omega, s^{\prime}, s\right)+\mathcal{D}_{5}\left(p, \zeta, \omega, s^{\prime}, s\right)\right]-\int_{s_{t h}}^{\infty} d s \\
& \left.\quad \times \rho_{B}(s, \zeta) \bar{\sigma}_{S}\left(s^{\prime}, \zeta, s_{t h}^{\prime}\right) \mathcal{D}_{3}\left(p, \zeta, \omega, s^{\prime}, s\right)\right\} .
\end{aligned}
$$

where

$$
\begin{aligned}
\mathcal{D}_{1}\left(p, \zeta, \omega, s^{\prime}\right)= & -i \frac{1}{\left(\zeta^{2}-p^{2}\right)} \\
& \times\left[\frac{\mathcal{I}_{1}\left(p, \omega, s^{\prime}\right)}{p^{2}}-\frac{\mathcal{I}_{1}\left(\zeta, \omega, s^{\prime}\right)}{\zeta^{2}}\right] \\
\mathcal{D}_{4}\left(p, \zeta, \omega, s^{\prime}, s\right)= & -i \frac{1}{\left(\zeta^{2}-p^{2}\right)} \\
& \times\left[\frac{\mathcal{I}_{4}\left(p, \omega, s^{\prime}, s\right)}{p^{2}}-\frac{\mathcal{I}_{4}\left(\zeta, \omega, s^{\prime}, s\right)}{\zeta^{2}}\right]
\end{aligned}
$$

$$
\begin{aligned}
\mathcal{D}_{4}^{\prime}\left(p, \zeta, \omega, s^{\prime}, s\right)= & -i \frac{1}{\left(\zeta^{2}-p^{2}\right)} \\
& \times\left[\frac{\mathcal{I}_{4}\left(p, \omega, s^{\prime}, s\right)}{\left(p^{2}-s+i \epsilon\right)}-\frac{\mathcal{I}_{4}\left(\zeta, \omega, s^{\prime}, s\right)}{\left(\zeta^{2}-s+i \epsilon\right)}\right] \\
\mathcal{D}_{5}\left(p, \zeta, \omega, s^{\prime}, s\right)= & -i \frac{1}{3\left(\zeta^{2}-p^{2}\right)} \\
& \times\left[\frac{\mathcal{I}_{5}\left(p, \omega, s^{\prime}, s\right)}{p^{2}\left(p^{2}-s+i \epsilon\right)}-\frac{\mathcal{I}_{5}\left(\zeta, \omega, s^{\prime}, s\right)}{\zeta^{2}\left(\zeta^{2}-s+i \epsilon\right)}\right]
\end{aligned}
$$

By exploiting Eq. (A.43), $\mathcal{D}_{1}$ can be evaluated as follows

$$
\mathcal{D}_{1}\left(p, \zeta, \omega, s^{\prime}\right)=-i \frac{1}{\left(\zeta^{2}-p^{2}\right)} \int_{0}^{1} d \xi \int \frac{d^{4} q}{(2 \pi)^{4}}
$$

Springer 


$$
\begin{aligned}
& \times\left\{\frac{\xi}{\left[q^{2}+\xi(1-\xi) p^{2}-\xi \omega-(1-\xi) s^{\prime}+i \epsilon\right]^{2}}\right. \\
& -\frac{\xi}{\left[q^{2}+\xi(1-\xi) \zeta^{2}-\xi \omega-(1-\xi) s^{\prime}+i \epsilon\right]^{2}} \\
& -\frac{4}{3} \int_{0}^{1-\xi} d t\left[\frac{q^{2}-(q \cdot p)^{2} / p^{2}}{\left[q^{2}+t(1-t) p^{2}-\xi \omega-t s^{\prime}+i \epsilon\right]^{3}}\right. \\
& \left.\left.-\frac{q^{2}-(q \cdot \zeta)^{2} / \zeta^{2}}{\left[q^{2}+t(1-t) \zeta^{2}-\xi \omega-t s^{\prime}+i \epsilon\right]^{3}}\right]\right\} \\
& =-\frac{1}{(4 \pi)^{2}} \int_{0}^{1} d v \int_{0}^{\infty} d y \frac{1}{\left[v \zeta^{2}+(1-v) p^{2}-y+i \epsilon\right]^{2}} \\
& \times \int_{0}^{1} d \xi\left\{\xi \Theta\left[y \xi(1-\xi)-\xi \omega-(1-\xi) s^{\prime}\right]\right.
\end{aligned}
$$

From Eq. (A.37), one gets

$$
\begin{aligned}
& \mathcal{D}_{4}\left(p, \zeta, \omega, s^{\prime}, s\right)=\frac{1}{(4 \pi)^{2}} \int_{0}^{1} d v \int_{0}^{\infty} d y \\
& \quad \times \frac{1}{\left\{v \zeta^{2}+(1-v) p^{2}-y+i \epsilon\right\}^{2}} \int_{0}^{1} d \xi \int_{0}^{1-\xi} d t \\
& \quad \times\left\{\frac{1}{(1-\xi)} \delta\left[y-\frac{\xi \omega+t s^{\prime}+(1-\xi-t) s}{\xi(1-\xi)}\right]\right. \\
& \left.\quad-\int_{0}^{1-\xi-t} \frac{d w}{\mathcal{A}_{4}(t, w)} \delta\left[y-\frac{\xi \omega+t s^{\prime}+w s}{\mathcal{A}_{4}(t, w)}\right]\right\},
\end{aligned}
$$

and

$$
\begin{aligned}
& \mathcal{D}_{4}^{\prime}\left(p, \zeta, \omega, s^{\prime}, s\right)=-\frac{1}{(4 \pi)^{2}} \int_{0}^{1} d v \int_{0}^{\infty} d y \\
& \quad \times \int_{0}^{1} d \xi \int_{0}^{1-\xi} d t \frac{y}{\left[v \zeta^{2}+(1-v) p^{2}-y+i \epsilon\right]^{2}} \\
& \quad \times\left\{\xi \Delta^{\prime}\left[y-s+\frac{s \xi(1-\xi)-\xi \omega-t s^{\prime}-(1-\xi-t) s}{\xi(1-\xi)}\right]\right. \\
& \left.\quad-\int_{0}^{1-\xi-t} d w \Delta^{\prime}\left[y-s+\frac{s \mathcal{A}_{4}(t, w)-\xi \omega-t s^{\prime}-w s}{\mathcal{A}_{4}(t, w)}\right]\right\} .
\end{aligned}
$$

(A.60)

Recalling that one has first to apply the dimensional regularization to $\mathcal{I}_{5}$, from Eq. (A.33) and using Eq. (A.46), and introducing one has

$\mathcal{D}_{5}\left(p, \zeta, \omega, s^{\prime}, s\right)=-i \frac{2}{3\left(\zeta^{2}-p^{2}\right)} \int_{0}^{1} d \xi \int_{0}^{1-\xi} d t$

$\times \int \frac{d^{4} q}{(2 \pi)^{4}}\left[\chi_{5}(q, p)-\chi_{5}(q, \zeta)\right]$

$$
\begin{aligned}
= & -\frac{1}{(4 \pi)^{2}} \int_{0}^{1} d v \int_{0}^{\infty} d y \\
& \times \frac{y}{\left[\left(v \zeta^{2}+(1-v) p^{2}\right)-y+i \epsilon\right]^{2}} \int_{0}^{1} d \xi \xi^{2} \int_{0}^{1-\xi} d t \\
& \times \Delta^{\prime}\left[y-s+\frac{s \xi(1-\xi)-\xi \omega-t s^{\prime}-(1-\xi-t) s}{\xi(1-\xi)}\right] .
\end{aligned}
$$

with

$$
\begin{aligned}
& \chi_{5}(q, p)=\frac{1}{p^{2}\left(p^{2}-s+i \epsilon\right)} \\
& \times \frac{4\left[q^{2} p^{2}-(q \cdot p)^{2}\right]-3 p^{2} q^{2}-3 p^{4} \xi^{2}}{\left[q^{2}+\xi(1-\xi) p^{2}-\xi \omega-t s^{\prime}-(1-\xi-t) s+i \epsilon\right]^{3}} .
\end{aligned}
$$

By using Eqs. (A.48), (A.58), (A.59), (A.60) and (A.61), one has

$$
\begin{aligned}
& \frac{\mathcal{A}_{R}(\zeta ; p)}{\zeta^{2}-p^{2}}=\frac{3 e_{R}^{2} Z_{1}(\zeta)}{(4 \pi)^{2}} \int_{0}^{1} d v \int_{0}^{\infty} d y \int_{0}^{\infty} d \omega \int_{0}^{1} d \xi \\
& \quad \times \int_{0}^{\infty} d s^{\prime} \frac{\bar{\sigma}_{\gamma}(\omega, \zeta, \zeta p)}{\left[\left(v \zeta^{2}+(1-v) p^{2}\right)-y+i \epsilon\right]^{2}} \\
& \quad \times\left\{\overline { \sigma } _ { V } ( s ^ { \prime } , \zeta , s _ { t h } ^ { \prime } ) \left[\left(\xi \Theta\left[y \xi(1-\xi)-\xi \omega-(1-\xi) s^{\prime}\right]\right.\right.\right. \\
& \left.\quad-\int_{0}^{1-\xi} d t \Theta\left[y t(1-t)-\xi \omega-t s^{\prime}\right]\right) \\
& +\int_{s_{t h}}^{\infty} d s \rho_{A}(s, \zeta)\left(\mathcal{C}_{A V}^{(0)}\left(\zeta, \omega, s, s^{\prime}, \xi, y\right)\right. \\
& \left.\left.+y \mathcal{C}_{A V}^{(1)}\left(\zeta, \omega, s, s^{\prime}, \xi, y\right)\right)\right]-\bar{\sigma}_{S}\left(s^{\prime}, \zeta, s_{t h}^{\prime}\right) \\
& \quad \times \int_{s_{t h}}^{\infty} d s \rho_{B}(s, \zeta) \int_{0}^{1-\xi} d t \int_{0}^{1-\xi-t} d w y \\
& \left.\quad \times \Delta^{\prime}\left[y-s+\frac{s \mathcal{A}_{4}(t, w)-\xi \omega-t s^{\prime}-w s}{\mathcal{A}_{4}(t, w)}\right]\right\}
\end{aligned}
$$

with $Z_{1}(\zeta)=Z_{1}(\zeta, \Lambda \rightarrow \infty)$,

$$
\begin{aligned}
\mathcal{C}_{A V}^{(0)}\left(\zeta, \omega, s, s^{\prime}, \xi, y\right)=\frac{\xi \Theta\left[y \xi(1-\xi)-\xi \omega-(1-\xi) s^{\prime}\right]}{\zeta^{2}-s+i \epsilon} \\
-\int_{0}^{1-\xi} d t \frac{\Theta\left[y t(1-t)-\xi \omega-t s^{\prime}\right]}{\zeta^{2}-s+i \epsilon} \\
+\int_{0}^{1-\xi} d t\left\{\frac{1}{(1-\xi)} \delta\left[y-\frac{\xi \omega+t s^{\prime}+(1-\xi-t) s}{\xi(1-\xi)}\right]\right. \\
\left.-\int_{0}^{1-\xi-t} \frac{d w}{\mathcal{A}_{4}(t, w)} \delta\left[y-\frac{\xi \omega+t s^{\prime}+w s}{\mathcal{A}_{4}(t, w)}\right]\right\}, \quad \text { (A.64) }
\end{aligned}
$$




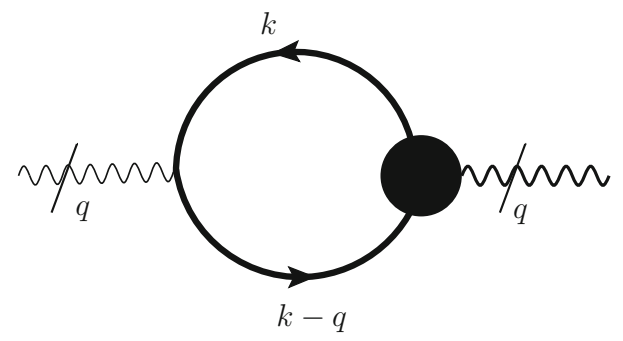

Fig. 9 The pictorial representation of the regularized photon selfenergy in Eq. (60), with the external legs amputated. The thick lines are the renormalized propagators of i) the fermion and antifermion pair and ii) the incoming photon, while the thin one is the free photon propagator. The full dot represents the renormalized interaction vertex

and

$$
\begin{aligned}
& \mathcal{C}_{A V}^{(1)}\left(\zeta, \omega, s, s^{\prime}, \xi, y\right)=\int_{0}^{1-\xi} d t\{(1+\xi) \xi \\
& \quad \times \Delta^{\prime}\left[y-s+\frac{s \xi(1-\xi)-\xi \omega-t s^{\prime}-(1-\xi-t) s}{\xi(1-\xi)}\right] \\
& \left.\quad-\int_{0}^{1-\xi-t} d w \Delta^{\prime}\left[y-s+\frac{s \mathcal{A}_{4}(t, w)-\xi \omega-t s^{\prime}-w s}{\mathcal{A}_{4}(t, w)}\right]\right\} .
\end{aligned}
$$

\section{Appendix B: DSE for the photon self-energy}

This Appendix is devoted to obtain the integral equation determining the renormalized photon self-energy. Equation (33). The initial step is given by the DSE for the regularized polarization tensor, Eq. (56), that we rewrite here for convenience, (the kinematical quantities are shown in Fig. 9)

$$
\begin{aligned}
& \Pi^{\mu \nu}(\zeta, \Lambda ; q)=-q^{2} T^{\mu v} \Pi(\zeta, \Lambda ; q)=-i \frac{Z_{1}(\zeta, \Lambda)}{Z_{3}(\zeta, \Lambda)} e_{R}^{2} \\
& \times \int_{\Lambda} \frac{d^{4} k}{(2 \pi)^{4}} \operatorname{Tr}\left\{\gamma^{\mu} S_{R}(\zeta, k) \Gamma_{R}^{v}(\zeta ; k, k-q) S_{R}(\zeta, k-q)\right\}
\end{aligned}
$$

where Eq. (A.2) has been used for the renormalized quantities. From Eq. (B.66) and the properties (59), it follows that the renormalized photon self-energy, Eq. (33) reads

$$
\Pi_{R}(\zeta ; q)=\left[\mathcal{T}_{P}(\zeta, \Lambda ; q)-\left.\mathcal{T}_{P}(\zeta, \Lambda ; q)\right|_{q^{2}=\zeta_{p}^{2}}\right]
$$

with

$$
\begin{aligned}
& \mathcal{T}_{P}(\zeta, \Lambda ; q)=-i Z_{1}(\zeta, \Lambda) \frac{4}{3} \frac{e_{R}^{2}}{q^{2}} \int_{s_{t h}} d s \\
& \quad \times \int_{s_{t h}^{\prime}} d s^{\prime} \int_{\Lambda} \frac{d^{4} k}{(2 \pi)^{4}} \frac{1}{k^{2}-s+i \epsilon} \\
& \quad \times \frac{1}{(k-q)^{2}-s^{\prime}+i \epsilon} \frac{\mathcal{P}_{\mu \nu}}{4} \operatorname{Tr}\left\{\gamma ^ { \mu } \left[\not k \bar{\sigma}_{V}\left(s, \zeta, s_{t h}\right)\right.\right.
\end{aligned}
$$

$$
\begin{aligned}
& \left.+\bar{\sigma}_{S}\left(s, \zeta, s_{t h}\right)\right] \Gamma_{R}^{v}(\zeta ; k, k-q) \\
& \left.\times\left[(\not k-\not \mid) \bar{\sigma}_{V}\left(s^{\prime}, \zeta, s_{t h}^{\prime}\right)+\bar{\sigma}_{S}\left(s^{\prime}, \zeta, s_{t h}^{\prime}\right)\right]\right\}
\end{aligned}
$$

where we have used: (i) the KL representation of the fermion propagator, Eq. (15), (ii) the definitions of $\bar{\sigma}_{V(S)}$ in Eq.(66). From the vertex contributions Eqs. (46), (52) and the relation $\gamma_{5} \epsilon^{\mu \alpha \nu \rho} \gamma_{\alpha}(k-q)_{\nu} k_{\rho}=\gamma_{5} \epsilon^{\mu \alpha \nu \rho} \gamma_{\alpha} k_{\nu} q_{\rho}$, one can define the relevant trace:

$$
\begin{aligned}
S p^{\mu \nu}= & \bar{\sigma}_{V}\left(s^{\prime}, \zeta, s_{t h}^{\prime}\right) \bar{\sigma}_{V}\left(s, \zeta, s_{t h}\right) T r_{1}^{\mu \nu} \\
& +\bar{\sigma}_{S}\left(s^{\prime}, \zeta, s_{t h}^{\prime}\right) \bar{\sigma}_{S}\left(s, \zeta, s_{t h}\right) T r_{2}^{\mu \nu} \\
& +\bar{\sigma}_{V}\left(s^{\prime}, \zeta, s_{t h}^{\prime}\right) \bar{\sigma}_{S}\left(s, \zeta, s_{t h}\right) \operatorname{Tr}_{3}^{\mu \nu} \\
& +\bar{\sigma}_{S}\left(s^{\prime}, \zeta, s_{t h}^{\prime}\right) \bar{\sigma}_{V}\left(s, \zeta, s_{t h}\right) \operatorname{Tr}_{4}^{\mu \nu}
\end{aligned}
$$

where

$$
\begin{aligned}
\operatorname{Tr}_{i}^{\mu \nu}= & \frac{1}{4} \operatorname{Tr}\left\{O _ { i } ^ { \mu } \left[\frac{\gamma^{v}}{2} F_{\mathcal{A}_{+}}(k, k-q, \zeta)\right.\right. \\
& -\frac{(2 \not k-\not \phi)(2 k-q)^{v}}{2} F_{\mathcal{A}_{-}}(k, k-q, \zeta) \\
& -(2 k-q)^{v} F_{\mathcal{B}}(k, k-q, \zeta)-\frac{1}{2}\left[q^{2} \gamma^{v}\right. \\
& \left.\left.\left.-q^{v} \phi+2 i \gamma^{5} \epsilon^{v \alpha \beta \rho} \gamma_{\alpha} k_{\beta} q_{\rho}\right] F_{\mathcal{A}_{-}}(k, k-q, \zeta)\right]\right\}
\end{aligned}
$$

with

$O_{1}^{\mu}=(\not k-\not k) \gamma^{\mu} \not k, \quad O_{2}^{\mu}=\gamma^{\mu}$,

$O_{3}^{\mu}=(\not k-\not k) \gamma^{\mu}, \quad O_{4}^{\mu}=\gamma^{\mu} \not k$.

Performing the traces (recall that $\epsilon^{0123}=1$ ), one has for the first trace

$$
\begin{aligned}
T_{1}^{\mu \nu} & =\frac{\tau_{1}^{\mu \nu}}{2} F_{\mathcal{A}_{+}}\left(k, k_{p}, \zeta\right) \\
& -\frac{\left(k+k_{p}\right)^{\nu}}{2}\left[k_{p}^{\mu} k \cdot\left(k+k_{p}\right)-\left(k+k_{p}\right)^{\mu} k_{p} \cdot k\right. \\
+ & \left.k^{\mu} k_{p} \cdot\left(k+k_{p}\right)\right] F_{\mathcal{A}_{-}}\left(k, k_{p}, \zeta\right)-[\frac{1}{2} \underbrace{\left(q^{2} \tau_{1}^{\mu \nu}-\tau_{2}^{\mu \nu}\right)}_{T 8} \\
& \underbrace{\left.-g^{\mu \nu}\left(k^{2} q^{2}-(k \cdot q)^{2}\right)-\tau_{3}^{\mu \nu} k \cdot q+\tau_{4}^{\mu \nu}\right]}_{T_{3}} \\
\times & F_{\mathcal{A}_{-}}\left(k, k_{p}, \zeta\right),
\end{aligned}
$$

where the following notation has been introduced for getting a more compact expression

$$
\begin{aligned}
k_{p} & =k-q \\
\tau_{1}^{\mu \nu} & =k_{p}^{\mu} k^{v}+k_{p}^{v} k^{\mu}-g^{\mu v} k_{p} \cdot k, \\
\tau_{2}^{\mu \nu} & =q^{v}\left(k_{p}^{\mu} k \cdot q-q^{\mu} k_{p} \cdot k+k^{\mu} k_{p} \cdot q\right), \\
\tau_{3}^{\mu \nu} & =q^{\mu} k^{v}+q^{v} k^{\mu}, \\
\tau_{4}^{\mu \nu} & =q^{\mu} q^{v} k^{2}+k^{\mu} k^{v} q^{2} .
\end{aligned}
$$


Moreover, the contributions from the transverse vertexes, i.e. $T_{3}$ and $T_{8}$, have been properly emphasized.

After introducing

$\mathcal{K}^{\mu \nu}=k^{\mu} k^{\nu}-\frac{1}{2}\left(q^{\mu} k^{\nu}+q^{\nu} k^{\mu}\right)$,

one can obtain:

$$
\begin{aligned}
\operatorname{Tr}_{1}^{\mu \nu}= & {\left[\mathcal{K}^{\mu \nu}-\frac{g^{\mu \nu}}{2}\left(k^{2}-q \cdot k\right)\right]\left[F_{\mathcal{A}_{+}}(k, k-q, \zeta)\right.} \\
& \left.+\left(2 k \cdot q-q^{2}\right) F_{\mathcal{A}_{-}}(k, k-q, \zeta)\right] \\
& -2\left[\tau_{4}^{\mu \nu}+k^{2} \mathcal{K}^{\mu \nu}-k \cdot q \tau_{3}^{\mu \nu}\right] F_{\mathcal{A}_{-}}(k, k-q, \zeta) \\
& +g^{\mu \nu}\left(k^{2} q^{2}-(k \cdot q)^{2}\right) F_{\mathcal{A}_{-}}(k, k-q, \zeta),
\end{aligned}
$$

The remaining traces are given by

$$
\begin{aligned}
\operatorname{Tr}_{2}^{\mu \nu} & =\frac{g^{\mu \nu}}{2} F_{\mathcal{A}_{+}}(k, k-q, \zeta)-\left[(2 k-q)^{\mu}(2 k-q)^{v}\right. \\
& +\underbrace{\left.\left(q^{2} g^{\mu \nu}-q^{\mu} q^{v}\right)\right]}_{T_{3}} \\
& =\frac{g^{\mu \nu}}{2}\left[F_{\mathcal{A}_{+}}(k, k-q, \zeta)-q^{2} F_{\mathcal{A}_{-}}(k, k-q, \zeta)\right] \\
& -2 \mathcal{K}^{\mu \nu} F_{\mathcal{A}_{-}}(k, k-q, \zeta), \\
\operatorname{Tr}_{3}^{\mu \nu} & =-(k-q)^{\mu}(2 k-q)^{v} F_{\mathcal{B}}(k, k-q, \zeta), \\
& =-\left[2 \mathcal{K}^{\mu \nu}+q^{\mu}\left(q^{v}-k^{v}\right)\right] F_{\mathcal{B}}(k, k-q, \zeta), \\
\operatorname{Tr}_{4}^{\mu \nu} & =-k^{\mu}(2 k-q)^{v} F_{\mathcal{B}}(k, k-q, \zeta) \\
= & -\left[2 \mathcal{K}^{\mu \nu}+k^{v} q^{\mu}\right] F_{\mathcal{B}}(k, k-q, \zeta) .
\end{aligned}
$$

Saturating the tensor $S p^{\mu \nu}$ with $\mathcal{P}^{\mu \nu}$ one gets for $T_{P}$ (recall that $g_{\mu \nu} \mathcal{P}^{\mu \nu}=0$ )

$$
\begin{aligned}
\mathcal{T}_{P} & (\zeta, \Lambda ; q)=-i Z_{1}(\zeta, \Lambda) \frac{4}{3} \frac{e_{R}^{2}}{q^{2}} \int_{s_{t h}} d s \int_{s_{t h}^{\prime}} d s^{\prime} \\
\quad & \times \int_{\Lambda} \frac{d^{4} k}{(2 \pi)^{4}} \frac{1}{k^{2}-s+i \epsilon} \frac{1}{(k-q)^{2}-s^{\prime}+i \epsilon} \\
\quad \times & \int_{V}\left(s^{\prime}, \zeta, s_{t h}^{\prime}\right) \bar{\sigma}_{V}\left(s, \zeta, s_{t h}\right)\left[\left(\mathcal{R}_{1}(k, q)+3 k \cdot q\right)\right. \\
& \times 2\left(1-\mathcal{A}_{R}(\zeta, k)\right)-2\left[(k-q)^{2}\left(\mathcal{R}_{1}(k, q)+3 k \cdot q\right)\right. \\
& \left.\left.-2 k^{2} q^{2}+2(k \cdot q)^{2}\right] F_{\mathcal{A}}(k, k-q, \zeta)\right]-2 \bar{\sigma}_{S}\left(s^{\prime}, \zeta, s_{t h}^{\prime}\right) \\
& \times \bar{\sigma}_{S}\left(s, \zeta, s_{t h}\right)\left(\mathcal{R}_{1}(k, q)+3 k \cdot q\right) F_{\mathcal{A}_{-}}(k, k-q, \zeta) \\
& -\bar{\sigma}_{S}\left(s^{\prime}, \zeta, s_{t h}^{\prime}\right) \bar{\sigma}_{V}\left(s, \zeta, s_{t h}\right)\left[2 \mathcal{R}_{1}(k, q)+3 k \cdot q\right. \\
& \left.+3\left(2(k \cdot q)-q^{2}\right)\right] F_{\mathcal{B}}(k, k-q, \zeta)-\bar{\sigma}_{V}\left(s^{\prime}, \zeta, s_{t h}^{\prime}\right)
\end{aligned}
$$

$$
\left.\times \bar{\sigma}_{S}\left(s, \zeta, s_{t h}\right)\left[2 \mathcal{R}_{1}(k, q)+3 k \cdot q\right] F_{\mathcal{B}}(k, k-q, \zeta)\right\}
$$

with

$\mathcal{R}_{1}(k, q)=k^{2}-4 \frac{(k \cdot q)^{2}}{q^{2}}$

and (see Eq. (A.19), with $p \rightarrow k-q$ )

$$
\begin{aligned}
2\left(1-\mathcal{A}_{R}(\zeta, k)\right)= & F_{\mathcal{A}_{+}}(k, k-q, \zeta) \\
& -\left(2 k \cdot q-q^{2}\right) F_{\mathcal{A}_{-}}(k, k-q, \zeta)
\end{aligned}
$$

As in the case of the fermion self-energy (see Appendix A), inserting the expressions of $\mathcal{F}_{A_{+}}, \mathcal{F}_{A_{-}}$and $\mathcal{F}_{B}$ in terms of the NWFS (see Eq. (47)) one can write

$$
\begin{aligned}
\mathcal{T}_{P} & (\zeta, \Lambda ; q)=-i Z_{1}(\zeta, \Lambda) \frac{4}{3} \frac{e_{R}^{2}}{q^{2}} \int_{s_{t h}}^{\infty} d s \int_{0}^{\infty} d s^{\prime} \\
& \times\left\{\overline { \sigma } _ { V } ( s ^ { \prime } , \zeta , s _ { t h } ^ { \prime } ) \overline { \sigma } _ { V } ( s , \zeta , s _ { t h } ) \left[2 \mathcal{I}_{6}\left(q, s, s^{\prime}\right)\right.\right. \\
& \times\left(1+\int_{s_{t h}}^{\infty} d \omega \frac{\rho_{A}(\omega, \zeta)}{\left(\zeta^{2}-\omega+i \epsilon\right)}\right)+2 \int_{s_{t h}}^{\infty} d \omega \\
& \left.\times \rho_{A}(\omega, \zeta)\left(\omega \mathcal{I}_{7}\left(q, s, s^{\prime}, \omega\right)-2 \mathcal{I}_{8}\left(q, s, s^{\prime}, \omega\right)\right)\right] \\
& +2 \bar{\sigma}_{S}\left(s^{\prime}, \zeta, s_{t h}^{\prime}\right) \bar{\sigma}_{S}\left(s, \zeta, s_{t h}\right) \\
& \times \int_{s_{t h}}^{\infty} d \omega \rho_{A}(\omega, \zeta) \mathcal{I}_{7}\left(q, s, s^{\prime}, \omega\right) \\
& +\left[\bar{\sigma}_{S}\left(s^{\prime}, \zeta, s_{t h}^{\prime}\right) \bar{\sigma}_{V}\left(s, \zeta, s_{t h}\right)\right. \\
& \left.+\bar{\sigma}_{V}\left(s^{\prime}, \zeta, s_{t h}^{\prime}\right) \bar{\sigma}_{S}\left(s, \zeta, s_{t h}\right)\right] \int_{s_{t h}}^{\infty} d \omega \rho_{B}(\omega, \zeta) \\
& \times\left(2 \mathcal{I}_{7}\left(q, s, s^{\prime}, \omega\right)-3 \mathcal{I}_{9}\left(q, s, s^{\prime}, \omega\right)\right) \\
& +3 \bar{\sigma}_{S}\left(s^{\prime}, \zeta, s_{t h}^{\prime}\right) \bar{\sigma}_{V}\left(s, \zeta, s_{t h}\right) \int_{s_{t h}}^{\infty} d \omega \rho_{B}(\omega, \zeta) \\
& \left.\times\left(2 \mathcal{I}_{9}\left(q, s, s^{\prime}, \omega\right)-q^{2} \mathcal{I}_{10}\left(q, s, s^{\prime}, \omega\right)\right)\right\}
\end{aligned}
$$

where the integrals $\mathcal{I}_{i}$, are defined as follows

$$
\begin{aligned}
& \mathcal{I}_{6}\left(q, s, s^{\prime}\right)=\int_{\Lambda} \frac{d^{4} k}{(2 \pi)^{4}} \frac{\left[k^{2}-4 \frac{(k \cdot q)^{2}}{q^{2}}+3 k \cdot q\right]}{k^{2}-s+i \epsilon} \\
& \quad \times \frac{1}{(k-q)^{2}-s^{\prime}+i \epsilon} \\
& \mathcal{I}_{7}\left(q, s, s^{\prime}, \omega\right) \\
& \quad \int_{\Lambda} \frac{d^{4} k}{(2 \pi)^{4}} \frac{\left[k^{2}-4 \frac{(k \cdot q)^{2}}{q^{2}}+3 k \cdot q\right]}{k^{2}-s+i \epsilon} \\
& \quad \times \frac{1}{\left[(k-q)^{2}-s^{\prime}+i \epsilon\right]\left[k^{2}-\omega+i \epsilon\right]\left[(k-q)^{2}-\omega+i \epsilon\right]}
\end{aligned}
$$




$$
\begin{aligned}
\mathcal{I}_{8}\left(q, s, s^{\prime}, \omega\right)=\int_{\Lambda} \frac{d^{4} k}{(2 \pi)^{4}} \frac{\left[k^{2} q^{2}-(k \cdot q)^{2}\right]}{k^{2}-s+i \epsilon} \\
\quad \times \frac{1}{\left[(k-q)^{2}-s^{\prime}+i \epsilon\right]\left[k^{2}-\omega+i \epsilon\right]\left[(k-q)^{2}-\omega+i \epsilon\right]}
\end{aligned}
$$

$$
\begin{aligned}
\mathcal{I}_{9}\left(q, s, s^{\prime}, \omega\right)=\int_{\Lambda} \frac{d^{4} k}{(2 \pi)^{4}} \frac{(k \cdot q)}{k^{2}-s+i \epsilon} \\
\quad \times \frac{1}{\left[(k-q)^{2}-s^{\prime}+i \epsilon\right]\left[k^{2}-\omega+i \epsilon\right]\left[(k-q)^{2}-\omega+i \epsilon\right]}
\end{aligned}
$$

$$
\begin{aligned}
\mathcal{I}_{10}\left(q, s, s^{\prime}, \omega\right)=\int_{\Lambda} \frac{d^{4} k}{(2 \pi)^{4}} \frac{1}{k^{2}-s+i \epsilon} \\
\quad \times \frac{1}{\left[(k-q)^{2}-s^{\prime}+i \epsilon\right]\left[k^{2}-\omega+i \epsilon\right]\left[(k-q)^{2}-\omega+i \epsilon\right]}
\end{aligned}
$$

with $s, s^{\prime}, \omega \geq 0$. Recall that the external momentum $q^{2}$ is chosen spacelike, for the sake of simplicity in the formal elaboration.

Notice that $\mathcal{I}_{6}$ presents an apparent quadratically divergence, as expected. Therefore, we exploit dimensional regularization, like the integral $\mathcal{I}_{0}$ (see Eq. (A.43)), and obtain

$$
\begin{aligned}
\mathcal{I}_{6}\left(q, s, s^{\prime}\right)= & i \frac{3 q^{2}}{(4 \pi)^{2}} \int_{0}^{1} d \xi \int_{0}^{\infty} d x \\
& \times \frac{\xi(1-\xi) x}{\left[-x+\xi(1-\xi) q^{2}-\xi s^{\prime}-(1-\xi) s+i \epsilon\right]^{2}}
\end{aligned}
$$

that has a logarithmic divergence, harmless once we subtract the corresponding integral evaluated at $\zeta_{p}^{2}$ (see Eq. (B.67)).

The other integrals are convergent and after applying the Feynman parametrization and the change of variable $p_{0} \rightarrow$ $i p_{4}$, one gets

$$
\begin{gathered}
\mathcal{I}_{7}\left(q, s, s^{\prime}, \omega\right)=i \frac{3}{(4 \pi)^{2}} q^{2} \int_{0}^{1} d \xi \int_{0}^{1-\xi} d v \\
\times \int_{0}^{1-\xi-v} d w \frac{\mathcal{A}_{4}(v, w)}{\left[D_{p h}^{a}\left(q, s^{\prime}, s, v, w, \xi, \omega\right)+i \epsilon\right]^{2}}
\end{gathered}
$$

$$
\begin{aligned}
& \mathcal{I}_{8}\left(q, s, s^{\prime}, \omega\right)=i \frac{3 q^{2}}{2(4 \pi)^{2}} \int_{0}^{1} d \xi \int_{0}^{1-\xi} d v \\
& \times \int_{0}^{1-\xi-v} d w \frac{1}{\left[D_{p h}^{a}\left(q, s^{\prime}, s, v, w, \xi, \omega\right)+i \epsilon\right]}
\end{aligned}
$$

$\mathcal{I}_{9}\left(q, s, s^{\prime}, \omega\right)=i \frac{q^{2}}{(4 \pi)^{2}} \int_{0}^{1} d \xi \int_{0}^{1-\xi} d v$

$$
\begin{gathered}
\times \int_{0}^{1-\xi-v} d w \frac{(v+w)}{\left[D_{p h}^{a}\left(q, s^{\prime}, s, v, w, \xi, \omega\right)+i \epsilon\right]^{2}}, \\
\mathcal{I}_{10}\left(q, s, s^{\prime}, \omega\right)=i \frac{1}{(4 \pi)^{2}} \int_{0}^{1} d \xi \int_{0}^{1-\xi} d v \\
\times \int_{0}^{1-\xi-v} d w \frac{\left.D_{p h}^{a}\left(q, s^{\prime}, s, v, w, \xi, \omega\right)+i \epsilon\right]^{2}}{\left[D_{p}\right.},
\end{gathered}
$$

where

$$
\begin{aligned}
D_{p h}^{a}\left(q, s^{\prime}, s, v, w, \xi, \omega\right)= & \mathcal{A}_{4}(v, w) q^{2}-v s^{\prime}-(\xi+w) \omega \\
& -(1-v-\xi-w) s
\end{aligned}
$$

By using Eq. (B.79) and the definition in Eq. (B.67), the photon self-energy reads (see Eq. (38))

$$
\begin{aligned}
\Pi_{R}(\zeta ; q)=Z_{1}(\zeta, \Lambda) \frac{4}{3} e_{R}^{2}\left(\zeta_{p}^{2}-q^{2}\right) \int_{0}^{\infty} d s \int_{0}^{\infty} d s^{\prime} \\
\quad \times\left\{\overline { \sigma } _ { V } ( s ^ { \prime } , \zeta , s _ { t h } ^ { \prime } ) \overline { \sigma } _ { V } ( s , \zeta , s _ { t h } ) \left[2 \mathcal{D}_{6}\left(q, \zeta_{p}, s, s^{\prime}\right)\right.\right. \\
\quad \times\left(1+\int_{s_{t h}}^{\infty} d \omega \frac{\rho_{A}(\omega, \zeta)}{\left(\zeta^{2}-\omega+i \epsilon\right)}\right)+2 \int_{s_{t h}}^{\infty} d \omega \rho_{A}(\omega, \zeta) \\
\left.\quad \times\left(\omega \mathcal{D}_{7}\left(q, \zeta_{p}, s, s^{\prime}, \omega\right)-2 \mathcal{D}_{8}\left(q, \zeta_{p}, s, s^{\prime}, \omega\right)\right)\right] \\
+2 \bar{\sigma}_{S}\left(s^{\prime}, \zeta, s_{t h}^{\prime}\right) \bar{\sigma}_{S}\left(s, \zeta, s_{t h}\right) \\
\times \int_{s_{t h}}^{\infty} d \omega \rho_{A}(\omega, \zeta) \mathcal{D}_{7}\left(q, \zeta_{p}, s, s^{\prime}, \omega\right) \\
+\bar{\sigma}_{S}\left(s^{\prime}, \zeta, s_{t h}^{\prime}\right) \bar{\sigma}_{V}\left(s, \zeta, s_{t h}\right) \\
\times \int_{s_{t h}}^{\infty} d \omega \rho_{B}(\omega, \zeta) \mathcal{D}_{7,9}\left(q, \zeta_{p}, s, s^{\prime}, \omega\right) \\
+\bar{\sigma}_{V}\left(s^{\prime}, \zeta, s_{t h}^{\prime}\right) \bar{\sigma}_{S}\left(s, \zeta, s_{t h}\right) \int_{s_{t h}}^{\infty} d \omega \rho_{B}(\omega, \zeta) \\
\left.\left.\times\left(\mathcal{D}_{7,9}\left(q, \zeta_{p}, s, s^{\prime}, \omega\right)+\mathcal{D}_{9,10}\left(q, \zeta_{p}, s, s^{\prime}, \omega\right)\right)\right)\right\}
\end{aligned}
$$

where

$$
\begin{aligned}
\mathcal{D}_{n}\left(q, \zeta_{p}, s, s^{\prime}\right)=-i \frac{1}{\left(\zeta_{p}^{2}-q^{2}\right)} \\
\times\left[\frac{\mathcal{I}_{n}\left(q, s, s^{\prime}\right)}{q^{2}}-\frac{\mathcal{I}_{n}\left(\zeta_{p}, s, s^{\prime}\right)}{\zeta_{p}^{2}}\right],
\end{aligned}
$$

with $n=6,7,8,9,10$, and

$$
\begin{aligned}
& \mathcal{D}_{7,9}\left(q, \zeta_{p}, s, s^{\prime}, \omega\right)=2 \mathcal{D}_{7}\left(q, \zeta_{p}, s, s^{\prime}, \omega\right) \\
& \quad-3 \mathcal{D}_{9}\left(q, \zeta_{p}, s, s^{\prime}, \omega\right) \\
& \mathcal{D}_{9,10}\left(q, \zeta_{p}, s, s^{\prime}, \omega\right)=6 \mathcal{D}_{9}\left(q, \zeta_{p}, s, s^{\prime}, \omega\right) \\
& \quad-3 \mathcal{D}_{10}\left(q, \zeta_{p}, s, s^{\prime}, \omega\right) .
\end{aligned}
$$


Eur. Phys. J. C (2021) 81:34

Page 27 of $35 \quad 34$

The explicit expressions of $\mathcal{D}_{6}, \mathcal{D}_{7}$ and $\mathcal{D}_{8}$, are

$$
\begin{aligned}
\mathcal{D}_{6}\left(q, \zeta_{p}, s, s^{\prime}\right)=\frac{6}{(4 \pi)^{2}} \int_{0}^{1} d \xi \xi^{2}(1-\xi)^{2} \int_{0}^{1} d v \\
\times \int_{0}^{\infty} d x \frac{x}{\left[D_{p h}^{b}\left(q, s^{\prime}, s, v, x, \xi, \omega, \zeta_{p}\right)+i \epsilon\right]^{3}} \\
=\frac{3}{(4 \pi)^{2}} \int_{0}^{1} d \xi \xi(1-\xi) \int_{0}^{1} d v \int_{0}^{\infty} d y \\
\times \frac{\delta\left[y-\left(\xi s^{\prime}+(1-\xi) s\right) /(\xi(1-\xi))\right]}{\left[(1-v) q^{2}+v \zeta_{p}^{2}-y+i \epsilon\right]} \\
=-\frac{3}{(4 \pi)^{2}} \int_{0}^{1} d \xi \xi(1-\xi) \int_{0}^{1} d v \int_{0}^{\infty} d y \\
\times \frac{\Theta\left[y \xi(1-\xi)-\xi s^{\prime}-(1-\xi) s\right]}{\left[(1-v) q^{2}+v \zeta_{p}^{2}-y+i \epsilon\right]^{2}}
\end{aligned}
$$

with

$$
\begin{gathered}
D_{p h}^{b}\left(q, s^{\prime}, s, v, x, \xi, \omega, \zeta_{p}\right)=-x+\xi(1-\xi) q^{2} \\
-\xi s^{\prime}-(1-\xi) s+v \xi(1-\xi)\left(\zeta_{p}^{2}-q^{2}\right) \\
\mathcal{D}_{7}\left(q, \zeta_{p}, s, s^{\prime}, \omega\right)=\frac{6}{(4 \pi)^{2}} \int_{0}^{1} d \xi \int_{0}^{1-\xi} d v \int_{0}^{1-\xi-v} d w \\
\quad \times \int_{0}^{1} d t \frac{\mathcal{A}_{4}^{2}(v, w)}{\left[D_{p h}^{c}\left(q, s^{\prime}, s, v, w, t, \xi, \omega, \zeta_{p}\right)+i \epsilon\right]^{3}} \\
=-\frac{3}{(4 \pi)^{2}} \int_{0}^{1} d \xi \int_{0}^{1-\xi} d v \int_{0}^{1-\xi-v} \frac{d w}{\mathcal{A}_{4}(v, w)} \\
\times \int_{0}^{1} d t \int_{0}^{\infty} d y \frac{\frac{\partial}{\partial y} \delta\left[y-\mathcal{A}_{7}\left(s, s^{\prime}, \omega, v, \xi, w\right)\right]}{\left[(1-t) q^{2}+t \zeta_{p}^{2}-y+i \epsilon\right]}
\end{gathered}
$$

with

$$
\begin{aligned}
D_{p h}^{c}\left(q, s^{\prime}, s, v, w, t, \xi, \omega, \zeta_{p}\right)=D_{p h}^{a}\left(q, s^{\prime}, s, v, w, \xi, \omega\right) \\
\quad+t v(1-v)\left(\zeta_{p}^{2}-q^{2}\right), \\
\mathcal{D}_{8}\left(q, \zeta_{p}, s, s^{\prime}, \omega\right)=\frac{3}{2(4 \pi)^{2}} \int_{0}^{1} d \xi \int_{0}^{1-\xi} d v \\
\quad \times \int_{0}^{1-\xi-v} d w \mathcal{A}_{4}(v, w) \int_{0}^{1} d t \\
\quad \times \frac{1}{\left[D_{p h}^{c}\left(q, s^{\prime}, s, v, w, \xi, \omega\right)+i \epsilon\right]^{2}} \\
=\frac{3}{2(4 \pi)^{2}} \int_{0}^{1} d \xi \int_{0}^{1-\xi} d v \int_{0}^{1-\xi-v} \frac{d w}{\mathcal{A}_{4}(v, w)} \\
\quad \times \int_{0}^{1} d t \int_{0}^{\infty} d y \frac{\delta\left[y-\mathcal{A}_{7}\left(s, s^{\prime}, \omega, v, \xi, w\right)\right]}{\left[(1-t) q^{2}+t \zeta_{p}^{2}-y+i \epsilon\right]}
\end{aligned}
$$

In view of the application of the uniqueness theorem for extracting $\rho_{\gamma}$, it is useful to apply an integration by part while evaluating $\mathcal{D}_{9}$ and $\mathcal{D}_{10}$. One gets

$$
\begin{aligned}
& \mathcal{D}_{9}\left(q, \zeta_{p}, s, s^{\prime}, \omega\right)=\frac{2}{(4 \pi)^{2}} \int_{0}^{1} d \xi \int_{0}^{1-\xi} d v \\
& \times \int_{0}^{1-\xi-v} d w \frac{(v+w)}{\mathcal{A}_{4}^{2}(v, w)} \\
& \times \int_{0}^{1} d t \int_{0}^{\infty} d y \frac{\delta\left[y-\mathcal{A}_{7}\left(s, s^{\prime}, \omega, v, \xi, w\right)\right]}{\left[(1-t) q^{2}+t \zeta_{p}^{2}-y+i \epsilon\right]^{3}} \\
&=-\frac{1}{(4 \pi)^{2}} \int_{0}^{1} d \xi \int_{0}^{1-\xi} d v \int_{0}^{1-\xi-v} d w \frac{(v+w)}{\mathcal{A}_{4}^{2}(v, w)} \\
& \times \int_{0}^{1} d t \int_{0}^{\infty} d y \frac{\frac{\partial}{\partial y} \delta\left[y-\mathcal{A}_{7}\left(s, s^{\prime}, \omega, v, \xi, w\right)\right]}{\left[(1-t) q^{2}+t \zeta_{p}^{2}-y+i \epsilon\right]^{2}}
\end{aligned}
$$

and

$$
\begin{aligned}
\mathcal{D}_{10}\left(q, \zeta_{p}, s, s^{\prime}, \omega\right)=\frac{2}{(4 \pi)^{2}} \int_{0}^{1} d \xi \int_{0}^{1-\xi} d v \\
\times \int_{0}^{1-\xi-v} \frac{d w}{\mathcal{A}_{4}^{2}(v, w)} \int_{0}^{1} d t \int_{0}^{\infty} d y \\
\times \frac{\delta\left[y-\mathcal{A}_{7}\left(s, s^{\prime}, \omega, v, \xi, w\right)\right]}{\left[(1-t) q^{2}+t \zeta_{p}^{2}-y+i \epsilon\right]^{3}} \\
=-\frac{1}{(4 \pi)^{2}} \int_{0}^{1} d \xi \int_{0}^{1-\xi} d v \int_{0}^{1-\xi-v} \frac{d w}{\mathcal{A}_{4}^{2}(v, w)} \\
\times \int_{0}^{1} d t \int_{0}^{\infty} d y \frac{\frac{\partial}{\partial y} \delta\left[y-\mathcal{A}_{7}\left(s, s^{\prime}, \omega, v, \xi, w\right)\right]}{\left[(1-t) q^{2}+t \zeta_{p}^{2}-y+i \epsilon\right]^{2}},
\end{aligned}
$$

with

$$
\begin{aligned}
& \mathcal{A}_{7}\left(s, s^{\prime}, \omega, v, \xi, w\right) \\
& =\frac{v s^{\prime}+(\xi+w) \omega+(1-\xi-v-w) s}{\mathcal{A}_{4}(v, w)} .
\end{aligned}
$$

Finally, one has

$$
\begin{gathered}
\mathcal{D}_{7,9}\left(q, \zeta_{p}, s, s^{\prime}, \omega\right)=-\frac{3}{(4 \pi)^{2}} \int_{0}^{1} d \xi \int_{0}^{1-\xi} d v \\
\times \int_{0}^{1-\xi-v} d w \frac{v+w}{\mathcal{A}_{4}^{2}(v, w)}[1-2(v+w)] \int_{0}^{1} d t \\
\times \int_{0}^{\infty} d y \frac{\frac{\partial}{\partial y} \delta\left[y-\mathcal{A}_{7}\left(s, s^{\prime}, \omega, v, \xi, w\right)\right]}{\left[(1-t) q^{2}+t \zeta_{p}^{2}-y+i \epsilon\right]^{2}}
\end{gathered}
$$

Springer 
and

$$
\begin{gathered}
\mathcal{D}_{9,10}\left(q, \zeta_{p}, s, s^{\prime}, \omega\right)=\frac{3}{(4 \pi)^{2}} \int_{0}^{1} d \xi \int_{0}^{1-\xi} d v \\
\times \int_{0}^{1-\xi-v} \frac{d w}{\mathcal{A}_{4}^{2}(v, w)}[1-2(v+w)] \int_{0}^{1} d t \\
\times \int_{0}^{\infty} d y \frac{\frac{\partial}{\partial y} \delta\left[y-\mathcal{A}_{7}\left(s, s^{\prime}, \omega, v, \xi, w\right)\right]}{\left[(1-t) q^{2}+t \zeta_{p}^{2}-y+i \epsilon\right]^{2}}
\end{gathered}
$$

Before obtaining the integral equation fulfilled by the NWF $\rho_{\gamma}$, it is useful to show that the last two terms in Eq. (B.90) are equal. As a matter of fact, one can recast the following term in a different form by reintroducing the fourth Feynman parameter, i.e.

$$
\begin{aligned}
& \int_{s_{t h}}^{\infty} d s \int_{0}^{\infty} d s^{\prime} \bar{\sigma}_{S}\left(s^{\prime}, \zeta, s_{t h}^{\prime}\right) \bar{\sigma}_{V}\left(s, \zeta, s_{t h}\right) \int_{0}^{1} d \xi \\
& \quad \times \int_{0}^{1} d v \int_{0}^{1} d w \int_{0}^{1} d u(v+w) \\
& \quad \times \delta(1-u-w-v-\xi) \frac{1-2(v+w)}{(v+w)^{2}(1-v-w)^{2}} \\
& \quad \times \frac{\partial}{\partial y} \delta\left[y-\frac{\left.v s^{\prime}+(\xi+w) \omega\right)+u s}{(v+w)(1-v-w)}\right]
\end{aligned}
$$

Then, re-naming the following variables: (i) $s \rightarrow s^{\prime}$, (ii) $v \rightarrow u$ and (iii) $\xi \rightarrow w$ and (iv) by exploiting the delta function, one has $u+\xi=1-v-w$ and can write

$$
\begin{aligned}
\int_{0}^{\infty} & d s \int_{0}^{\infty} d s^{\prime} \bar{\sigma}_{S}\left(s, \zeta, s_{t h}\right) \bar{\sigma}_{V}\left(s^{\prime}, \zeta, s_{t h}^{\prime}\right) \int_{0}^{1} d \xi \\
& \times \int_{0}^{1} d v \int_{0}^{1} d w \int_{0}^{1} d u(1-v-w) \\
& \times \delta(1-v-\xi-u-w) \frac{1-2(1-v-w)}{(1-v-w)^{2}(v+w)^{2}} \\
& \times \frac{\partial}{\partial y} \delta\left[y-\frac{u s+(w+\xi) \omega)+v s^{\prime}}{(1-v-w)(v+w)}\right] \\
= & -\int_{0}^{\infty} d s \int_{0}^{\infty} d s^{\prime} \bar{\sigma}_{S}\left(s, \zeta, s_{t h}\right) \bar{\sigma}_{V}\left(s^{\prime}, \zeta, s_{t h}^{\prime}\right) \int_{0}^{1} d \xi \\
& \times \int_{0}^{1} d v \int_{0}^{1} d w \int_{0}^{1} d u(1-v-w) \\
& \times \delta(1-v-\xi-u-w) \frac{1-2(v+w)}{(1-v-w)^{2}(v+w)^{2}} \\
& \times \frac{\partial}{\partial y} \delta\left[y-\frac{\left.v s^{\prime}+(w+\xi) \omega\right)+u s}{(1-v-w)(v+w)}\right] \quad Q . E . D .
\end{aligned}
$$

After introducing the uniqueness theorem in Eq. (B.90), one gets for $\rho_{\gamma}$ (see Eq. (38))

$\Theta\left(y-s_{t h}^{p}\right) \rho_{\gamma}(y, \zeta)=-\lim _{\Lambda \rightarrow \infty} Z_{1}(\zeta, \Lambda) \frac{e_{R}^{2}}{(2 \pi)^{2}} \int_{0}^{\infty} d s$

$$
\begin{aligned}
& \times \int_{0}^{\infty} d s^{\prime} \int_{0}^{1} d \xi\left\{\bar{\sigma}_{V}\left(s^{\prime}, \zeta, s_{t h}^{\prime}\right) \bar{\sigma}_{V}\left(s, \zeta, s_{t h}\right)\right. \\
& \times\left[2 \xi(1-\xi) \Theta\left[y \xi(1-\xi)-\xi s^{\prime}-(1-\xi) s\right]\right. \\
& \times\left(1+\int_{s_{t h}}^{\infty} d \omega \frac{\rho_{A}(\omega, \zeta, \Lambda)}{\left(\zeta^{2}-\omega+i \epsilon\right)}\right)+2 \int_{s_{t h}}^{\infty} d \omega \rho_{A}(\omega, \zeta) \\
& \times \int_{0}^{1-\xi} d v \int_{0}^{1-\xi-v} \frac{d w}{\mathcal{A}_{4}(v, w)} \\
& \times\left(\omega \frac{\partial}{\partial y} \delta\left[y-\mathcal{A}_{7}\left(s, s^{\prime}, \omega, v, \xi, w\right)\right]\right. \\
& \left.\left.+\delta\left[y-\mathcal{A}_{7}\left(s, s^{\prime}, \omega, v, \xi, w\right)\right]\right)\right]+\bar{\sigma}_{S}\left(s^{\prime}, s_{t h}^{\prime} \zeta\right) \\
& \times \bar{\sigma}_{S}\left(s, \zeta, s_{t h}\right) \int_{s_{t h}}^{\infty} d \omega \rho_{A}(\omega, \zeta) \int_{0}^{1-\xi} d v \\
& \times \int_{0}^{1-\xi-v} \frac{d w}{\mathcal{A}_{4}(v, w)} \frac{\partial}{\partial y} \delta\left[y-\mathcal{A}_{7}\left(s, s^{\prime}, \omega, v, \xi, w\right)\right] \\
& +2 \bar{\sigma}_{S}\left(s^{\prime}, \zeta, s_{t h}^{\prime}\right) \bar{\sigma}_{V}\left(s, \zeta, s_{t h}\right) \int_{s_{t h}}^{\infty} d \omega \rho_{B}(\omega, \zeta) \\
& \times \int_{0}^{1-\xi} d v \int_{0}^{1-\xi-v} d w(v+w) \frac{1-2(v+w)}{\mathcal{A}_{4}^{2}(v, w)} \\
& \left.\times \frac{\partial}{\partial y} \delta\left[y-\mathcal{A}_{7}\left(s, s^{\prime}, \omega, v, \xi, w\right)\right]\right\}
\end{aligned}
$$

with

$$
\begin{aligned}
& Z_{1}(\zeta),=\lim _{\Lambda \rightarrow \infty} Z_{1}(\zeta, \Lambda) \\
& \mathcal{A}_{4}=(t+w)(1-t-w) \\
& \mathcal{A}_{7}\left(s, s^{\prime}, \omega, v, \xi, w\right) \\
& \quad=\frac{\left[v s^{\prime}+(\xi+w) \omega+(1-\xi-v-w) s\right]}{\mathcal{A}_{4}(v, w)} .
\end{aligned}
$$

\section{Appendix C: First iteration}

This Appendix is devoted to present a first analytic result obtained by iterating one time the coupled system we have obtained.

The inputs are given by the zeroth-order NWFs $\rho_{A}, \rho_{B}$ and $\rho_{\gamma}$, i.e.

$\rho_{A}^{(0)}(s, \zeta)=\rho_{B}^{(0)}(s, \zeta)=\rho_{\gamma}^{(0)}(s, \zeta)=0$,

Hence, the KL weights of the fermion and photon propagators (see Eqs. (23), (43) and (66)) read

$$
\begin{aligned}
\bar{\sigma}_{V}^{(0)}(s, \zeta) & =\delta\left(s-m^{2}(\zeta)\right), \\
\bar{\sigma}_{S}^{(0)}(s, \zeta) & =m(\zeta) \delta\left(s-m^{2}(\zeta)\right), \\
\bar{\sigma}_{\gamma}^{(0)}\left(s, \zeta, \zeta_{p}\right) & =\delta\left(s-\zeta_{p}^{2}\right),
\end{aligned}
$$


and the renormalization constants become (see Eq. (12))

$Z_{2}^{(0)}=1+\int_{s_{t h}}^{\infty} d s \frac{\rho_{A}^{(0)}(s, \zeta)}{\zeta^{2}-s+i \epsilon}=1=Z_{1}^{(0)}$

By inserting the tree-level expressions, Eq. (C.104), in Eq. (68) one obtains the first iteration for $\rho_{A}$, viz

$$
\begin{aligned}
\Theta(y & \left.-s_{t h}\right) \rho_{A}^{(1)}(y, \zeta)=\frac{3}{(4 \pi)^{2}} e_{R}^{2} \int_{0}^{\infty} d \omega \delta\left(\omega-\zeta_{p}^{2}\right) \\
& \times \int_{0}^{1} d \xi \int_{0}^{\infty} d s^{\prime} \delta\left(s^{\prime}-m^{2}(\zeta)\right) \\
& \times\left[\xi \Theta\left[y \xi(1-\xi)-\xi \omega-(1-\xi) s^{\prime}\right]\right. \\
& \left.-\int_{0}^{1-\xi} d t \Theta\left[y t(1-t)-\xi \omega-t s^{\prime}\right]\right] \\
= & \frac{3 e_{R}^{2}}{(4 \pi)^{2}} \int_{0}^{1} d \xi\left\{\xi \Theta\left[y \xi(1-\xi)-(1-\xi) m^{2}(\zeta)-\xi \zeta_{p}^{2}\right]\right. \\
& \left.-\int_{0}^{1-\xi} d t \Theta\left[y t(1-t)-t m^{2}(\zeta)-\xi \zeta_{p}^{2}\right]\right\} \\
= & \frac{3 e_{R}^{2}}{(4 \pi)^{2}}\left\{\int_{0}^{1} d \xi \xi \Theta\left[\xi(1-\xi) y-(1-\xi) m^{2}(\zeta)-\xi \zeta_{p}^{2}\right]\right. \\
& -\int_{0}^{1} d \xi \int_{0}^{1} d t^{\prime} \Theta\left(t^{\prime}-\xi\right) \\
& \left.\times \Theta\left[t^{\prime}\left(1-t^{\prime}\right) y-\left(1-t^{\prime}\right) m^{2}(\zeta)-\xi \zeta_{p}^{2}\right]\right\} . \quad(\mathrm{C} .107)
\end{aligned}
$$

Notice that the two theta functions imply also

$$
\xi y-m^{2}(\zeta) \geq 0, \quad t^{\prime} y-m^{2}(\zeta) \geq 0 .
$$

The second integral is usefully manipulated as follows

$$
\begin{aligned}
\int_{0}^{1} d \xi \int_{0}^{1} d t^{\prime} \Theta\left(t^{\prime}-\xi\right) \\
\quad \times \Theta\left[t^{\prime}\left(1-t^{\prime}\right) y-\left(1-t^{\prime}\right) m^{2}(\zeta)-\xi \zeta_{p}^{2}\right] \\
=\int_{0}^{1} d t^{\prime} \Theta\left[t^{\prime} y-m^{2}(\zeta)\right] \int_{0}^{1} d \xi \\
\quad \times\left\{\Theta\left(t^{\prime}-\xi\right) \Theta\left[t^{\prime}\left(1-t^{\prime}\right) y-\left(1-t^{\prime}\right) m^{2}(\zeta)-t^{\prime} \zeta_{p}^{2}\right]\right. \\
\quad+\Theta\left[t^{\prime}\left(1-t^{\prime}\right) y-\left(1-t^{\prime}\right) m^{2}(\zeta)-\xi \zeta_{p}^{2}\right] \\
\left.\quad \times \Theta\left\{t^{\prime} \zeta_{p}^{2}-\left[t^{\prime}\left(1-t^{\prime}\right) y-\left(1-t^{\prime}\right) m^{2}(\zeta)\right]\right\}\right\} \\
=\int_{0}^{1} d t^{\prime} \Theta\left[t^{\prime} y-m^{2}(\zeta)\right] \\
\quad \times\left\{t^{\prime} \Theta\left[t^{\prime}\left(1-t^{\prime}\right) y-\left(1-t^{\prime}\right) m^{2}(\zeta)-t^{\prime} \zeta_{p}^{2}\right]\right. \\
\quad+\frac{t^{\prime}\left(1-t^{\prime}\right) y-\left(1-t^{\prime}\right) m^{2}(\zeta)}{\zeta_{p}^{2}}
\end{aligned}
$$

$$
\left.\times \Theta\left[t^{\prime} \zeta_{p}^{2}-\left(t^{\prime}\left(1-t^{\prime}\right) y-\left(1-t^{\prime}\right) m^{2}(\zeta)\right)\right]\right\},
$$

where the two contributions are obtained by exploiting the two sets of inequalities

$$
\begin{aligned}
& \frac{t^{\prime}\left(1-t^{\prime}\right) y-\left(1-t^{\prime}\right) m^{2}(\zeta)}{\zeta_{p}^{2}} \geq t^{\prime} \geq \xi \\
& t^{\prime} \geq \frac{t^{\prime}\left(1-t^{\prime}\right) y-\left(1-t^{\prime}\right) m^{2}(\zeta)}{\zeta_{p}^{2}} \geq \xi
\end{aligned}
$$

Both sets are generated by the constraints on the variable $\xi$ in the first line of (C.109).

Recollecting the above results one obtains the following expression of $\rho_{A}^{(1)}$

$$
\begin{gathered}
\Theta\left(y-s_{t h}\right) \rho_{A}^{(1)}(y, \zeta)=\frac{3}{(4 \pi)^{2}} e_{R}^{2} \Theta\left(y-m^{2}(\zeta)\right) \\
\quad \times \int_{m^{2}(\zeta) / y}^{1} d t^{\prime} \frac{t^{\prime 2} y-t^{\prime}\left(y+m^{2}(\zeta)\right)+m^{2}(\zeta)}{\zeta_{p}^{2}} \\
\quad \times \Theta\left[t^{\prime 2} y-t^{\prime}\left(y+m^{2}(\zeta)-\zeta_{p}^{2}\right)+m^{2}(\zeta)\right]
\end{gathered}
$$

The constraints imposed by the theta function on $t^{\prime}$ can be obtained from the solutions of the second-order equation, that read

$$
\begin{aligned}
t_{ \pm}^{\prime}= & \frac{1}{2 y}\left[y+m^{2}(\zeta)-\zeta_{p}^{2}\right. \\
& \left. \pm \sqrt{\left[y-m^{2}(\zeta)-\zeta_{p}^{2}\right]^{2}-4 m^{2}(\zeta) \zeta_{p}^{2}}\right]
\end{aligned}
$$

It is important to notice that both real and complexconjugated solutions are allowed, due to the presence of IRregulator $\zeta_{p}^{2}$ in the discriminant. The complex-conjugated solutions lead to an IR-dependent contribution in $\rho_{A}^{(1)}$ that properly vanishes in the limit $\zeta_{p} \rightarrow 0$ matching the constraint expected from the lhs of Eq. (C.111). Notably, this term guarantees the continuity of $\rho_{A}$ and therefore of the KällénLehman weights, when approaching the physical threshold $y=m^{2}(\zeta)$.

The real positive solutions $t_{ \pm}^{\prime}$ are obtained when

$$
y \geq y_{+}=\left[m(\zeta)+\zeta_{p}\right]^{2} \text { or } y_{-}=\left[m(\zeta)-\zeta_{p}\right]^{2} \geq y,
$$

with also $y-\zeta_{p}^{2}+m^{2}(\zeta) \geq 0$. The constraint $y \geq m^{2}(\zeta)$ in Eq. (C.111) excludes $y_{-}$and one remains with $\Theta[y-(m+$ $\left.\zeta_{p}\right)^{2}$. In this case, one can easily show that the real solutions fulfill

$1 \geq t_{ \pm}^{r} \geq \frac{m^{2}(\zeta)}{y}$ 
Therefore

$t^{\prime} \in\left[\frac{m^{2}(\zeta)}{y}, t_{-}^{r}\right] \cup\left[t_{+}^{r}, 1\right]$.

The discriminant is negative, when

$\left[m(\zeta)+\zeta_{p}\right]^{2} \geq y \geq\left[m(\zeta)-\zeta_{p}\right]^{2}$,

and $t^{\prime}$ does not have any constraint, i.e. $t^{\prime} \in\left[m^{2}(\zeta) / y, 1\right]$. Moreover, taking into account $\Theta[y-m(\zeta)]$ one remains with $\left[m(\zeta)+\zeta_{p}\right]^{2} \geq y \geq m^{2}(\zeta)$, that generates an IR-dependent term with no impact for $\zeta_{p}^{2} \rightarrow 0$.

In conclusion, $\rho_{A}^{(1)}$ is given by

$$
\begin{aligned}
\Theta(y & \left.-s_{t h}\right) \rho_{A}^{(1)}(y, \zeta)=-\frac{3 e_{R}^{2}}{(4 \pi)^{2} \zeta_{p}^{2}} \Theta\left(y-m^{2}(\zeta)\right) \\
& \times\left\{\Theta\left[\left[m(\zeta)+\zeta_{p}\right]^{2}-y\right] \frac{\left(y-m^{2}(\zeta)\right)^{3}}{6 y^{2}}\right. \\
& +\Theta\left[y-\left[m(\zeta)+\zeta_{p}\right]^{2}\right]\left[\frac{\left(y-m^{2}(\zeta)\right)^{3}}{6 y^{2}}\right. \\
& \left.\left.+\left.\frac{2 t^{\prime 3} y-3 t^{\prime 2}\left(y+m^{2}(\zeta)\right)+6 m^{2}(\zeta) t^{\prime}}{6}\right|_{t_{-}^{\prime}} ^{t_{+}^{\prime}}\right]\right\} \\
= & -\frac{e_{R}^{2}}{2(4 \pi)^{2}} \frac{1}{\zeta_{p}^{2} y^{2}} \Theta\left(y-m^{2}(\zeta)\left\{\Theta\left[y-\left[m(\zeta)+\zeta_{p}\right]^{2}\right]\right.\right. \\
& \times\left(y-m^{2}(\zeta)\right)^{3}\left[1-f\left(y, \zeta, \zeta_{p}^{2}\right)\right] \\
+ & \left.\Theta\left[\left[m(\zeta)+\zeta_{p}\right]^{2}-y\right]\left(y-m^{2}(\zeta)\right)^{3}\right\}
\end{aligned}
$$

where

$$
\begin{aligned}
f\left(y, \zeta, \zeta_{p}^{2}\right)= & \sqrt{1-\zeta_{p}^{2} \frac{2 y+2 m^{2}(\zeta)-\zeta_{p}^{2}}{\left(y-m^{2}(\zeta)\right)^{2}}} \\
& \times\left[1+\zeta_{p}^{2} \frac{y+m^{2}(\zeta)-2 \zeta_{p}^{2}}{\left(y-m^{2}(\zeta)\right)^{2}}\right]
\end{aligned}
$$

with $y>\left[m(\zeta)+\zeta_{p}\right]^{2}$. To complete our analysis, let us consider $\Theta\left(y-s_{t h}\right) \rho_{A}^{(1)}(y, \zeta)$ for $\zeta_{p} \rightarrow 0$. In particular, one remains with the following limit

$$
\lim _{\zeta_{p} \rightarrow 0} \frac{1}{\zeta_{p}^{2}}\left[1-f\left(y, \zeta, \zeta_{p}^{2}\right)\right]=0
$$

since

$$
f\left(y, \zeta, \zeta_{p}^{2}\right) \sim 1+\frac{\zeta_{p}^{4}}{2} f^{\prime \prime}(0, y)
$$

Therefore one gets

$$
\begin{aligned}
& \lim _{\zeta_{p} \rightarrow 0} \Theta\left[y-\left(m(\zeta)+\zeta_{p}\right)^{2}\right] \rho_{A}^{(1)}(y, \zeta)=0 \\
& \lim _{y \rightarrow \infty} \Theta\left[y-\left(m(\zeta)+\zeta_{p}\right)^{2}\right] \rho_{A}^{(1)}(y, \zeta)=0
\end{aligned}
$$

Starting from Eq. (72) and repeating analogous steps one has for $\rho_{B}^{(1)}$

$$
\begin{aligned}
\Theta(y & \left.-s_{t h}\right) \rho_{B}^{(1)}(y, \zeta)=-\frac{3}{(4 \pi)^{2}} e_{R}^{2} m(\zeta) \\
& \times \int_{0}^{\infty} d \omega \delta\left(\omega-\zeta_{p}^{2}\right) \int_{0}^{1} d \xi \int_{0}^{\infty} d s^{\prime} \delta\left(s^{\prime}-m^{2}(\zeta)\right) \\
& \times \Theta\left[y \xi(1-\xi)-\xi \omega-(1-\xi) s^{\prime}\right]=-\frac{3}{(4 \pi)^{2}} e_{R}^{2} m(\zeta) \\
& \times \int_{0}^{1} d \xi \Theta\left[y \xi(1-\xi)-\xi\left(\zeta_{p}^{2}-m^{2}(\zeta)\right)-m^{2}(\zeta)\right] \\
= & -\frac{3}{(4 \pi)^{2}} e_{R}^{2} m(\zeta) \Theta\left[y-\left[m(\zeta)+\zeta_{p}\right]^{2}\right]\left(\xi_{+}-\xi_{-}\right) \\
= & \frac{3 e_{R}^{2}}{(4 \pi)^{2}} m(\zeta) \Theta\left[y-\left[m(\zeta)+\zeta_{p}\right]^{2}\right] \\
& \times \frac{1}{y} \sqrt{\left[y-\left(m^{2}(\zeta)+\zeta_{p}^{2}\right)\right]^{2}-4 m^{2}(\zeta) \zeta_{p}^{2}} \quad \text { (C.120) }
\end{aligned}
$$

with $\xi_{ \pm}$given in Eq. (C.112) after changing $t^{\prime} \rightarrow \xi$. Differently from $\rho_{A}^{(1)}(y, \zeta)$, this time no IR-dependent issue is present. For completeness, the following relevant limits have to considered.

$$
\begin{aligned}
& \lim _{\zeta_{p} \rightarrow 0} \rho_{B}^{(1)}(y, \zeta) \\
& \quad=-\frac{3}{(4 \pi)^{2}} e_{R}^{2} m(\zeta) \Theta\left[y-m(\zeta)^{2}\right] \frac{y-m^{2}(\zeta)}{y} \\
& \lim _{y \rightarrow \infty} \rho_{B}^{(1)}(y, \zeta)=-\frac{3}{(4 \pi)^{2}} e_{R}^{2} m(\zeta)
\end{aligned}
$$

It should be emphasized that the regularized $\mathcal{B}_{Z}(\zeta, \Lambda ; p)$, Eq. (7), obtained from the above $\rho_{B}^{(1)}$ and by taking into account the limits in Eq. (C.121), shows the expected singular behavior in both IR and UV regions.

For $\rho_{\gamma}$ in Eq. (77), one gets the following first iteration

$$
\begin{aligned}
\Theta(y & \left.-s_{t h}^{p}\right) \rho_{\gamma}^{(1)}(y, \zeta)=-\frac{e_{R}^{2}}{(2 \pi)^{2}} \int_{0}^{\infty} d s \delta\left(s-m^{2}(\zeta)\right) \\
& \times \int_{0}^{\infty} d s^{\prime} \delta\left(s^{\prime}-m^{2}(\zeta)\right) \int_{0}^{1} d \xi 2 \xi(1-\xi) \\
& \times \Theta\left[y \xi(1-\xi)-\xi s^{\prime}-(1-\xi) s\right]=-\frac{2}{(2 \pi)^{2}} e_{R}^{2} \\
& \times \int_{0}^{1} d \xi \xi(1-\xi) \Theta\left[y \xi(1-\xi)-m^{2}(\zeta)\right]= \\
= & -\frac{e_{R}^{2}}{3(2 \pi)^{2}} \Theta(y) \Theta\left(y-4 m^{2}(\zeta)\right)\left(1+2 \frac{m^{2}(\zeta)}{y}\right) \\
& \times \sqrt{1-4 \frac{m^{2}(\zeta)}{y}}
\end{aligned}
$$

with

$\xi_{ \pm}=\frac{1}{2}\left(1 \pm \sqrt{1-4 \frac{m^{2}(\zeta)}{y}}\right)$ 
Let us recall that $s_{t h}^{p}=4 m^{2}(\zeta)$. Moreover

$$
\lim _{y \rightarrow \infty} \rho_{\gamma}^{(1)}(y, \zeta)=\frac{e_{R}^{2}}{3(2 \pi)^{2}}
$$

and therefore $Z_{3}^{(1)}$ is logarithmically divergent, (see Eq. (37)). The first-order photon self-energy, Eq. (38), is given by

$$
\begin{aligned}
\Pi_{R}^{(1)}\left(\zeta, q^{2}\right)= & \int_{s_{t h}^{p}}^{\infty} d s \frac{\left(\zeta_{p}^{2}-q^{2}\right) \rho_{\gamma}^{(1)}(s, \zeta)}{\left(q^{2}-s+i \epsilon\right)\left(\zeta_{p}^{2}-s+i \epsilon\right)} \\
= & -\frac{e_{R}^{2}}{3(2 \pi)^{2}} \int_{s_{t h}^{p}}^{\infty} d s \frac{\left(\zeta_{p}^{2}-q^{2}\right)\left[s+2 m^{2}(\zeta)\right]}{s\left(q^{2}-s+i \epsilon\right)\left(\zeta_{p}^{2}-s+i \epsilon\right)} \\
& \times \sqrt{1-4 \frac{m^{2}(\zeta)}{s}}
\end{aligned}
$$

Notice that the imaginary part of $\Pi_{R}^{(1)}\left(\zeta, q^{2}\right)$, when $q^{2}>$ $4 m^{2}(\zeta)$, coincides with the result that can be found in Ref [89].

\section{Appendix D: Formulas Summary}

For the sake of a quick focus on the main formal results have been obtained in the paper, in this Appendix we list the initial expressions useful for for a numerical calculations of KL weights in terms of NWFs.

The three NWFs $\rho_{A}, \rho_{B}$ and $\rho_{\gamma}$ fulfill the following integral equations

$$
\begin{aligned}
& \Theta\left(y-s_{t h}\right) \rho_{A}(y, \zeta)=\frac{3}{(4 \pi)^{2}} e_{R}^{2} \lim _{\Lambda \rightarrow \infty} Z_{1}(\zeta, \Lambda) \\
& \quad \times \int_{0}^{\infty} d \omega \bar{\sigma}_{\gamma}\left(\omega, \zeta, \zeta_{p}, \Lambda\right) \int_{0}^{1} d \xi \int_{0}^{\infty} d s^{\prime} \\
& \left\{\overline { \sigma } _ { V } ( s ^ { \prime } , \zeta , s _ { t h } ^ { \prime } , \Lambda ) \left[\xi \Theta\left(y \xi(1-\xi)-\xi \omega-(1-\xi) s^{\prime}\right)\right.\right. \\
& \left.\quad-\int_{0}^{1-\xi} d t \Theta\left(y t(1-t)-\xi \omega-t s^{\prime}\right)\right]+\bar{\sigma}_{V}\left(s^{\prime}, \zeta, s_{t h}^{\prime}, \Lambda\right) \\
& \quad \times\left[\int_{s_{t h}}^{\infty} d s \rho_{A}(s, \zeta, \Lambda) \mathcal{C}_{A V}^{(0)}\left(\zeta, \omega, s, s^{\prime}, \xi, y\right)\right. \\
& \left.\quad+y \int_{s_{t h}}^{\infty} d s \rho_{A}(s, \zeta, \Lambda) \mathcal{C}_{A V}^{(1)}\left(\zeta, \omega, s, s^{\prime}, \xi, y\right)\right] \\
& \quad-y \bar{\sigma}_{S}\left(s^{\prime}, \zeta, s_{t h}^{\prime}, \Lambda\right) \int_{0}^{1-\xi} d t \int_{0}^{1-\xi-t} d w \\
& \quad \times \int_{s_{t h}}^{\infty} d s \rho_{B}(s, \zeta, \Lambda) \\
& \quad \times \Delta^{\prime}\left[y-s+\frac{s \mathcal{A}_{4}(t, w)-\xi \omega-t s^{\prime}-w s}{\mathcal{A}(t, w)}\right. \\
& \Theta\left(y-s_{t h}\right) \rho_{B}(y, \zeta)=-\frac{3}{(4 \pi)^{2}} e_{R}^{2} \lim _{\Lambda \rightarrow \infty} Z_{1}(\zeta, \Lambda) \\
& \quad \times \int_{0}^{\infty} d \omega \bar{\sigma}_{\gamma}(\omega, \zeta, \zeta p, \Lambda) \int_{0}^{1} d \xi \int_{0}^{\infty} d s^{\prime}
\end{aligned}
$$

$$
\begin{aligned}
& \times\left\{\bar{\sigma}_{S}\left(s^{\prime}, \zeta, s_{t h}^{\prime}, \Lambda\right) \Theta\left[y \xi(1-\xi)-\xi \omega-(1-\xi) s^{\prime}\right]\right. \\
& +\bar{\sigma}_{S}\left(s^{\prime}, \zeta, s_{t h}^{\prime}, \Lambda\right) \\
& \times\left[\int_{s_{t h}}^{\infty} d s \rho_{A}(s, \zeta, \Lambda) \mathcal{C}_{A S}^{(0)}\left(\zeta, \omega, s, s^{\prime}, \xi, y\right)\right. \\
& \left.+y \int_{s_{t h}}^{\infty} d s \rho_{A}(s, \zeta, \Lambda) \mathcal{C}_{A S}^{(1)}\left(\zeta, \omega, s, s^{\prime}, \xi, y\right)\right] \\
& +y \bar{\sigma}_{V}\left(s^{\prime}, \zeta, s_{t h}^{\prime}, \Lambda\right) \int_{0}^{1-\xi} d t \int_{0}^{1-\xi-t} d w \\
& \times \int_{s_{t h}}^{\infty} d s \rho_{B}(s, \zeta, \Lambda) \\
& \left.\times \Delta^{\prime}\left[y-s+\frac{s \mathcal{A}_{4}(t, w)-\xi \omega-t s^{\prime}-w s}{\mathcal{A}_{4}(t, w)}\right]\right\}, \\
& \Theta\left(y-s_{t h}^{p}\right) \rho_{\gamma}(y, \zeta)=-\frac{e_{R}^{2}}{(2 \pi)^{2}} \lim _{\Lambda \rightarrow \infty} Z_{1}(\zeta, \Lambda) \int_{0}^{\infty} d s \\
& \times \int_{0}^{\infty} d s^{\prime} \int_{0}^{1} d \xi\left\{\bar{\sigma}_{V}\left(s^{\prime}, \zeta, s_{t h}^{\prime}, \Lambda\right) \bar{\sigma}_{V}\left(s, \zeta, s_{t h}, \Lambda\right)\right. \\
& \left.\times 2 \xi(1-\xi) \Theta\left[y \xi(1-\xi)-\xi s^{\prime}-(1-\xi) s\right)\right] \\
& \times\left(1+\int_{s_{t h}}^{\infty} d \omega \frac{\rho_{A}(\omega, \zeta, \Lambda)}{\left(\zeta^{2}-\omega+i \epsilon\right)}\right) \\
& +\int_{s_{t h}}^{\infty} d \omega \rho_{A}(\omega, \zeta, \Lambda) \mathcal{C}_{\gamma}\left(s, s^{\prime}, \xi, \omega\right) \\
& +2 \bar{\sigma}_{S}\left(s^{\prime}, \zeta, s_{t h}^{\prime}, \Lambda\right) \bar{\sigma}_{V}\left(s, \zeta, s_{t h}, \Lambda\right) \\
& \times \int_{s_{t h}}^{\infty} d \omega \rho_{B}(\omega, \zeta, \Lambda) \int_{0}^{1-\xi} d v \int_{0}^{1-\xi-v} d w(v+w) \\
& \left.\times \frac{1-2(v+w)}{\mathcal{A}_{4}^{2}(v, w)} \frac{\partial}{\partial y} \delta\left[y-\mathcal{A}_{7}\left(s, s^{\prime}, \omega, v, \xi, w\right)\right]\right\},
\end{aligned}
$$

with $\Delta^{\prime}, \mathcal{C}_{A V}^{(i)}, \mathcal{C}_{A S}^{(i)}$ and $\mathcal{C}_{\gamma}$ from Eqs. (69), (70), (71), (73), (74) and (78), and the following equations that relate the NWFs to the KL weights (see Eq. (23))

$$
\begin{aligned}
\sigma_{V}(\omega, \zeta) & =\frac{D_{I}\left[1-\left(\zeta^{2}-\omega\right)\left\langle\rho_{A}\right\rangle\right]-\rho_{A}(\omega, \zeta) D_{R}}{D_{R}^{2}+\pi^{2} D_{I}^{2}} \\
\sigma_{S}(\omega, \zeta) & =\frac{D_{I}\left[m(\zeta)+\left(\zeta^{2}-\omega\right)\left\langle\rho_{B}\right\rangle\right]+\rho_{B}(\omega, \zeta) D_{R}}{D_{R}^{2}+\pi^{2} D_{I}^{2}}
\end{aligned}
$$

where $\omega \geq s_{t h}=\left(m(\zeta)+\zeta_{p}\right)^{2}$, the notation $\left\langle\rho_{A, B}\right\rangle$ means

$$
\left\langle\rho_{A, B}\right\rangle=\mathrm{P} . \mathrm{V} . \int_{s_{t h}}^{\infty} d s \frac{\rho_{A, B}(s, \zeta)}{\left(p^{2}-s\right)\left(\zeta^{2}-s+i \epsilon\right)}
$$

and

$$
\begin{aligned}
D_{R}= & \omega\left[\left(1-\left(\zeta^{2}-\omega\right)\left\langle\rho_{A}\right\rangle\right)^{2}-\pi^{2} \rho_{A}^{2}(\omega, \zeta)\right] \\
& -\left[\left(m(\zeta)+\left(\zeta^{2}-\omega\right)\left\langle\rho_{B}\right\rangle\right)^{2}-\pi^{2} \rho_{B}^{2}(\omega, \zeta)\right], \\
D_{I}= & 2 \omega \rho_{A}(\omega, \zeta)\left[1-\left(\zeta^{2}-\omega\right)\left\langle\rho_{A}\right\rangle\right]
\end{aligned}
$$




$$
+2 \rho_{B}(\omega, \zeta)\left[m(\zeta)+\left(\zeta^{2}-\omega\right)\left\langle\rho_{B}\right\rangle\right]
$$

Moreover, one has (see Eq. (43))

$$
\begin{aligned}
\sigma_{\gamma}(\omega, \zeta)= & -\frac{1}{\left(\omega-\zeta_{p}^{2}\right)} \\
& \times \frac{\rho_{\gamma}(\omega, \zeta)}{\left[\left(1+\left(\zeta_{p}^{2}-\omega\right)\left\langle\rho_{\gamma}\right\rangle\right)^{2}+\pi^{2} \rho_{\gamma}^{2}(\omega, \zeta)\right]},
\end{aligned}
$$

with $\omega \geq \zeta_{p}^{2}$.

The fundamental renormalization constant $Z_{2}=Z_{1}$ is given by

$$
\lim _{\Lambda \rightarrow \infty} Z_{2}(\zeta, \Lambda)=1+\lim _{\Lambda \rightarrow \infty} \int_{s_{t h}}^{\infty} d s \frac{\rho_{A}(s, \zeta, \Lambda)}{\zeta^{2}-s+i \epsilon}
$$

\section{References}

1. E.E. Salpeter, H.A. Bethe, A relativistic equation for bound-state problems. Phys. Rev. 84, 1232 (1951). https://doi.org/10.1103/ PhysRev.84.1232

2. M. Gell-Mann, F. Low, Bound states in quantum field theory. Phys. Rev. 84, 350 (1951). https://doi.org/10.1103/PhysRev.84.350

3. F.J. Dyson, The S matrix in quantum electrodynamics. Phys. Rev. 75, 1736 (1949). https://doi.org/10.1103/PhysRev.75.1736

4. J.S. Schwinger, On the Green's functions of quantized fields. 1. Proc. Natl. Acad. Sci. 37, 452 (1951). https://doi.org/10.1073/ pnas.37.7.452

5. J.S. Schwinger, On the Green's functions of quantized fields. 2. Proc. Natl. Acad. Sci. 37, 455 (1951). https://doi.org/10.1073/ pnas.37.7.455

6. C.D. Roberts, A.G. Williams, Dyson-Schwinger equations and their application to hadronic physics. Prog. Part. Nucl. Phys. 33, 477 (1994). https://doi.org/10.1016/0146-6410(94)90049-3

7. R. Alkofer, L. von Smekal, The infrared behavior of QCD Green's functions: confinement dynamical symmetry breaking, and hadrons as relativistic bound states. Phys. Rep. 353, 281 (2001). https://doi.org/10.1016/S0370-1573(01)00010-2

8. P. Maris, C.D. Roberts, Dyson-Schwinger equations: a tool for hadron physics. Int. J. Mod. Phys. E 12, 297 (2003). https://doi. org/10.1142/S0218301303001326

9. A. Bashir, L. Chang, I.C. Cloët, B. El-Bennich, Y.X. Liu, C.D. Roberts, P.C. Tandy, Collective perspective on advances in Dyson-Schwinger equation QCD. Commun. Theor. Phys. 58, 79 (2012). https://doi.org/10.1088/0253-6102/58/1/16

10. G. Eichmann, H. Sanchis-Alepuz, R. Williams, R. Alkofer, C.S. Fischer, Baryons as relativistic three-quark bound states. Prog. Part. Nucl. Phys. 91, 1 (2016). https://doi.org/10.1016/j.ppnp. 2016.07.001

11. H. Sanchis-Alepuz, R. Williams, Recent developments in boundstate calculations using the Dyson-Schwinger and Bethe-Salpeter equations. Comput. Phys. Commun. 232, 1 (2018). https://doi. org/10.1016/j.cpc.2018.05.020

12. S.X. Qin, C.D. Roberts, Resolving the Bethe-Salpeter kernel (2020). arXiv:2009.13637

13. S.J. Brodsky, H.C. Pauli, S.S. Pinsky, Quantum chromodynamics and other field theories on the light cone. Phys. Rep. 301, 299 (1998). https://doi.org/10.1016/S0370-1573(97)00089-6

14. J. Vary, H. Honkanen, J. Li, P. Maris, S. Brodsky, A. Harindranath, G. de Teramond, P. Sternberg, E. Ng, C. Yang, Hamiltonian light- front field theory in a basis function approach. Phys. Rev. C 81, 035205 (2010). https://doi.org/10.1103/PhysRevC.81.035205

15. J. Lan, C. Mondal, S. Jia, X. Zhao, J.P. Vary, Pion and kaon parton distribution functions from basis light front quantization and QCD evolution. Phys. Rev. D 101(3), 034024 (2020). https://doi.org/10. 1103/PhysRevD.101.034024

16. X. Zhao, K. Fu, H. Zhao, J. Lan, C. Mondal, S. Xu, J.P. Vary, in 18th International Conference on Hadron Spectroscopy and Structure, pp. 624-631 (2020). https://doi.org/10.1142/ 9789811219313_0107

17. J.B. Kogut, L. Susskind, Hamiltonian formulation of Wilson's lattice gauge theories. Phys. Rev. D 11, 395 (1975). https://doi. org/10.1103/PhysRevD.11.395

18. I. Raychowdhury, J.R. Stryker, Loop, string, and hadron dynamics in SU(2) Hamiltonian lattice gauge theories. Phys. Rev. D 101(11), 114502 (2020). https://doi.org/10.1103/PhysRevD.101.114502

19. M. Bañuls et al., Simulating lattice gauge theories within quantum technologies. Eur. Phys. J. D 74(8), 165 (2020). https://doi.org/ 10.1140/epjd/e2020-100571-8

20. P.C. Tandy, Covariant QCD modeling of light meson physics. Prog. Part. Nucl. Phys. 50, 305 (2003). https://doi.org/10.1016/ S0146-6410(03)00024-3. [305 (2003)]

21. L. Chang, C.D. Roberts, Sketching the Bethe-Salpeter kernel. Phys. Rev. Lett. 103, 081601 (2009). https://doi.org/10.1103/ PhysRevLett.103.081601

22. S.X. Qin, L. Chang, Y.X. Liu, C.D. Roberts, S.M. Schmidt, Practical corollaries of transverse Ward-Green-Takahashi identities. Phys. Lett. B 722, 384 (2013). https://doi.org/10.1016/j.physletb. 2013.04.034

23. S.X. Qin, C.D. Roberts, S.M. Schmidt, Ward-Green-Takahashi identities and the axial-vector vertex. Phys. Lett. B 733, 202 (2014). https://doi.org/10.1016/j.physletb.2014.04.041

24. D. Binosi, L. Chang, J. Papavassiliou, S.X. Qin, C.D. Roberts, Symmetry preserving truncations of the gap and Bethe-Salpeter equations. Phys. Rev. D 93(9), 096010 (2016). https://doi.org/10. 1103/PhysRevD.93.096010

25. D. Binosi, L. Chang, J. Papavassiliou, S.X. Qin, C.D. Roberts, Natural constraints on the gluon-quark vertex. Phys. Rev. D 95(3), 031501 (2017). https://doi.org/10.1103/PhysRevD.95.031501

26. S.X. Qin, C. Chen, C. Mezrag, C.D. Roberts, Off-shell persistence of composite pions and kaons. Phys. Rev. C 97(1), 015203 (2018). https://doi.org/10.1103/PhysRevC.97.015203

27. A.C. Aguilar, J.C. Cardona, M.N. Ferreira, J. Papavassiliou, Quark gap equation with non-abelian Ball-Chiu vertex. Phys. Rev. D 98(1), 014002 (2018). https://doi.org/10.1103/PhysRevD. 98.014002

28. J. Glimm, A.M. Jaffe, Quantum Physics. A Functional Integral Point of View (Springer, Berlin, 1987)

29. S. Chatterjee, Yang-Mills for probabilists (2018). arXiv e-prints arXiv: 1803.01950

30. K. Osterwalder, R. Schrader, Axioms for Euclidean Green's functions. II [with an Appendix by Stephen Summers]. Commun. Math. Phys. 42(3), 281 (1975). https://projecteuclid.org: 443/euclid.cmp/1103899050

31. D. Shirkov, I. Solovtsov, Analytic model for the QCD running coupling with universal $\bar{\alpha}_{s}(0)$ value. Phys. Rev. Lett. 79, 1209 (1997). https://doi.org/10.1103/PhysRevLett.79.1209

32. B. Joó, J. Karpie, K. Orginos, A. Radyushkin, D. Richards, S. Zafeiropoulos, Parton distribution functions from Ioffe time pseudo-distributions. JHEP 12, 081 (2019). https://doi.org/10. 1007/JHEP12(2019)081

33. C. Chen, L. Chang, C.D. Roberts, S. Wan, H.S. Zong, Valencequark distribution functions in the kaon and pion. Phys. Rev. D 93(7), 074021 (2016). https://doi.org/10.1103/PhysRevD.93. 074021 
34. K.D. Bednar, I.C. Cloët, P.C. Tandy, Distinguishing quarks and gluons in pion and kaon parton distribution functions. Phys. Rev. Lett. 124(4), 042002 (2020). https://doi.org/10.1103/ PhysRevLett.124.042002

35. L. Chang, I.C. Cloët, J.J. Cobos-Martinez, C.D. Roberts, S.M. Schmidt, P.C. Tandy, Imaging dynamical chiral symmetry breaking: pion wave function on the light front. Phys. Rev. Lett. 110(13), 132001 (2013). https://doi.org/10.1103/PhysRevLett. 110.132001

36. F. Gao, L. Chang, Y.X. Liu, Bayesian extraction of the parton distribution amplitude from the Bethe-Salpeter wave function. Phys. Lett. B 770, 551 (2017). https://doi.org/10.1016/j.physletb. 2017.04.077

37. S.S. Xu, L. Chang, C.D. Roberts, H.S. Zong, Pion and kaon valence-quark parton quasidistributions. Phys. Rev. D 97(9), 094014 (2018). https://doi.org/10.1103/PhysRevD.97.094014

38. C.R. Ji, P. Maris, K(13) transition form-factors. Phys. Rev. D 64, 014032 (2001). https://doi.org/10.1103/PhysRevD.64.014032

39. D. Kekez, D. Klabučar, Pion observables calculated in Minkowski and Euclidean spaces from Ansatz quark propagators (2020). arXiv:2006.02326

40. A. Bashir, A. Kizilersu, M.R. Pennington, The nonperturbative three point vertex in massless quenched QED and perturbation theory constraints. Phys. Rev. D 57, 1242 (1998). https://doi.org/ 10.1103/PhysRevD.57.1242

41. C.S. Fischer, R. Alkofer, T. Dahm, P. Maris, Dynamical chiral symmetry breaking in unquenched $\operatorname{QED}(3)$. Phys. Rev. D 70, 073007 (2004). https://doi.org/10.1103/PhysRevD.70.073007

42. A. Kizilersu, M.R. Pennington, Building the full fermion-photon vertex of QED by imposing multiplicative renormalizability of the Schwinger-Dyson equations for the fermion and photon propagators. Phys. Rev. D 79, 125020 (2009). https://doi.org/10.1103/ PhysRevD.79.125020

43. A. Kizilersu, T. Sizer, A.G. Williams, Strongly-coupled unquenched QED4 propagators using Schwinger-Dyson equations. Phys. Rev. D 88, 045008 (2013). https://doi.org/10.1103/ PhysRevD.88.045008

44. S. Jia, M.R. Pennington, Exact solutions to the fermion propagator Schwinger-Dyson equation in Minkowski space with on-shell renormalization for quenched QED. Phys. Rev. D 96(3), 036021 (2017). https://doi.org/10.1103/PhysRevD.96.036021

45. M. Tanabashi et al., Review of particle physics. Phys. Rev. D 98(3), 030001 (2018). https://doi.org/10.1103/PhysRevD.98. 030001

46. A. Bashir, M. Pennington, Gauge independent chiral symmetry breaking in quenched QED. Phys. Rev. D 50, 7679 (1994). https:// doi.org/10.1103/PhysRevD.50.7679

47. A. Bashir, A. Raya, S. Sanchez-Madrigal, Chiral symmetry breaking and confinement beyond rainbow-ladder truncation. Phys. Rev. D 84, 036013 (2011). https://doi.org/10.1103/PhysRevD.84. 036013

48. M. Reenders, On the nontriviality of Abelian gauged NambuJona-Lasinio models in four-dimensions. Phys. Rev. D 62, 025001 (2000). https://doi.org/10.1103/PhysRevD.62.025001

49. D. Atkinson, J.C. Bloch, V. Gusynin, M. Pennington, M. Reenders, Strong QED with weak gauge dependence: critical coupling and anomalous dimension. Phys. Lett. B 329, 117 (1994). https:// doi.org/10.1016/0370-2693(94)90526-6

50. J.S. Ball, T.W. Chiu, Analytic properties of the vertex function in gauge theories. 1. Phys. Rev. D 22, 2542 (1980). https://doi.org/ 10.1103/PhysRevD.22.2542

51. K.I. Kondo, Longitudinal and transverse Ward-Takahashi identity, anomaly and Schwinger-Dyson equation. Int. J. Mod. Phys. A 12, 5651 (1997). https://doi.org/10.1142/S0217751X97002978

52. D.C. Curtis, M.R. Pennington, Truncating the Schwinger-Dyson equations: how multiplicative renormalizability and the Ward identity restrict the three point vertex in QED. Phys. Rev. D 42, 4165 (1990). https://doi.org/10.1103/PhysRevD.42.4165

53. A. Bashir, R. Bermudez, L. Chang, C.D. Roberts, Dynamical chiral symmetry breaking and the fermion-gauge-boson vertex. Phys. Rev. C 85, 045205 (2012). https://doi.org/10.1103/PhysRevC.85. 045205

54. A. Kizilersus, T. Sizer, M.R. Pennington, A.G. Williams, R. Williams, Dynamical mass generation in unquenched QED using the Dyson-Schwinger equations. Phys. Rev. D 91(6), 065015 (2015). https://doi.org/10.1103/PhysRevD.91.065015

55. N. Nakanishi, Partial-Wave Bethe-Salpeter equation. Phys. Rev. 130(3), 1230 (1963)

56. N. Nakanishi, A general survey of the theory of the Bethe-Salpeter equation. Prog. Theor. Phys. Suppl. 43, 1 (1969). https://doi.org/ 10.1143/PTPS.43.1

57. N. Nakanishi, Graph Theory and Feynman Integrals (Gordon and Breach, New York, 1971)

58. V. Sauli, J. Adam, Solving the Schwinger-Dyson equation for a scalar propagator in Minkowski space. Nucl. Phys. A 689, 467 (2001). https://doi.org/10.1016/S0375-9474(01)00884-3

59. V. Sauli, J. Adam Jr., Study of relativistic bound states for scalar theories in the Bethe-Salpeter and Dyson-Schwinger formalism. Phys. Rev. D 67, 085007 (2003). https://doi.org/10.1103/ PhysRevD.67.085007

60. V. Sauli, Minkowski solution of Dyson-Schwinger equations in momentum subtraction scheme. JHEP 02, 001 (2003). https://doi. org/10.1088/1126-6708/2003/02/001

61. V. Sauli, Running coupling and fermion mass in strong coupling QED(3+1). J. Phys. G 30, 739 (2004). https://doi.org/10.1088/ 0954-3899/30/6/005

62. V. Sauli, J. Adam Jr., P. Bicudo, Dynamical chiral symmetry breaking with integral Minkowski representations. Phys. Rev. D 75, 087701 (2007). https://doi.org/10.1103/PhysRevD.75. 087701

63. V. Sauli, Nakanishi integral representation for the quark-photon vertex (2019). arXiv:1909.03043

64. K. Kusaka, K. Simpson, A.G. Williams, Solving the BetheSalpeter equation for bound states of scalar theories in Minkowski space. Phys. Rev. D 56, 5071 (1997). https://doi.org/10.1103/ PhysRevD.56.5071

65. V.A. Karmanov, J. Carbonell, Solving Bethe-Salpeter equation in Minkowski space. Eur. Phys. J. A 27, 1 (2006). https://doi.org/10. 1140/epja/i2005-10193-0

66. J. Carbonell, V.A. Karmanov, Cross-ladder effects in BetheSalpeter and light-front equations. Eur. Phys. J. A 27, 11 (2006). https://doi.org/10.1140/epja/i2005-10194-y

67. J. Carbonell, V.A. Karmanov, Solving Bethe-Salpeter equation for two fermions in Minkowski space. Eur. Phys. J. A 46, 387 (2010). https://doi.org/10.1140/epja/i2010-11055-4

68. T. Frederico, G. Salmè, M. Viviani, Quantitative studies of the homogeneous Bethe-Salpeter equation in Minkowski space. Phys. Rev. D 89, 016010 (2014). https://doi.org/10.1103/ PhysRevD.89.016010

69. T. Frederico, G. Salmè, M. Viviani, Solving the inhomogeneous Bethe-Salpeter equation in Minkowski space: the zero-energy limit. Eur. Phys. J. C 75(8), 398 (2015). https://doi.org/10.1140/ epjc/s10052-015-3616-1

70. W. de Paula, T. Frederico, G. Salmè, M. Viviani, Advances in solving the two-fermion homogeneous Bethe-Salpeter equation in Minkowski space. Phys. Rev. D 94(7), 071901 (2016). https:// doi.org/10.1103/PhysRevD.94.071901

71. C. Gutierrez, V. Gigante, T. Frederico, G. Salmè, M. Viviani, L. Tomio, Bethe-Salpeter bound-state structure in Minkowski space. Phys. Lett. B 759, 131 (2016). https://doi.org/10.1016/j.physletb. 2016.05.066 
72. W. de Paula, T. Frederico, G. Salmè, M. Viviani, R. Pimentel, Fermionic bound states in Minkowski-space: light-cone singularities and structure. Eur. Phys. J. C 77(11), 764 (2017). https://doi. org/10.1140/epjc/s10052-017-5351-2

73. J.H.Alvarenga Nogueira, D. Colasante, V. Gherardi, T. Frederico, E. Pace, G. Salmè, Solving the Bethe-Salpeter Equation in Minkowski Space for a Fermion-scalar system. Phys. Rev. D 100(1), 016021 (2019). https://doi.org/10.1103/PhysRevD.100. 016021

74. V. Sauli, Solving the Bethe-Salpeter equation for a pseudoscalar meson in Minkowski space. J. Phys. G 35, 035005 (2008). https:// doi.org/10.1088/0954-3899/35/3/035005

75. V. Sauli, Pions and excited scalars in Minkowski space DSBSE formalism. Int. J. Theor. Phys. 54(11), 4131 (2015). https://doi. org/10.1007/s10773-015-2525-2

76. C.S. Mello, J.P.B.C. de Melo, T. Frederico, Minkowski space pion model inspired by lattice QCD running quark mass. Phys. Lett. B 766, 86 (2017). https://doi.org/10.1016/j.physletb.2016.12.058

77. T. Frederico, D.C. Duarte, W. de Paula, E. Ydrefors, S. Jia, P. Maris, Towards Minkowski space solutions of Dyson-Schwinger Equations through un-Wick rotation (2019). arXiv: 1905.00703

78. E. Ydrefors, J.H.Alvarenga Nogueira, V.A. Karmanov, T. Frederico, Solving the three-body bound-state Bethe-Salpeter equation in Minkowski space. Phys. Lett. B 791, 276 (2019). https:// doi.org/10.1016/j.physletb.2019.02.046

79. J. Carbonell, T. Frederico, V.A. Karmanov, Bound state equation for the Nakanishi weight function. Phys. Lett. B 769, 418 (2017). https://doi.org/10.1016/j.physletb.2017.04.016

80. L. Chang, C. Mezrag, H. Moutarde, C.D. Roberts, J. RodríguezQuintero, P.C. Tandy, Basic features of the pion valence-quark distribution function. Phys. Lett. B 737, 23 (2014). https://doi. org/10.1016/j.physletb.2014.08.009

81. C. Mezrag, L. Chang, H. Moutarde, C.D. Roberts, J. RodríguezQuintero, F. Sabatié, S.M. Schmidt, Sketching the pion's valencequark generalised parton distribution. Phys. Lett. B 741, 190 (2015). https://doi.org/10.1016/j.physletb.2014.12.027

82. F. Gao, L. Chang, Y.X. Liu, C.D. Roberts, S.M. Schmidt, Parton distribution amplitudes of light vector mesons. Phys. Rev. D 90(1), 014011 (2014). https://doi.org/10.1103/PhysRevD.90.014011

83. C. Shi, L. Chang, C.D. Roberts, S.M. Schmidt, P.C. Tandy, H.S. Zong, Flavour symmetry breaking in the kaon parton distribution amplitude. Phys. Lett. B 738, 512 (2014). https://doi.org/10.1016/ j.physletb.2014.07.057

84. C. Fanelli, E. Pace, G. Romanelli, G. Salmè, M. Salmistraro, Pion generalized parton distributions within a fully covariant constituent quark model. Eur. Phys. J. C 76(5), 253 (2016). https:// doi.org/10.1140/epjc/s10052-016-4101-1

85. C. Mezrag, J. Segovia, L. Chang, C.D. Roberts, Parton distribution amplitudes: revealing correlations within the proton and Roper. Phys. Lett. B 783, 263 (2018). https://doi.org/10.1016/j.physletb. 2018.06.062

86. N. Chouika, C. Mezrag, H. Moutarde, J. Rodríguez-Quintero, A Nakanishi-based model illustrating the covariant extension of the pion GPD overlap representation and its ambiguities. Phys. Lett. B 780, 287 (2018). https://doi.org/10.1016/j.physletb.2018.02.070

87. N. Chouika, C. Mezrag, H. Moutarde, J. Rodríguez-Quintero, Covariant Extension of the GPD overlap representation at low Fock states. Eur. Phys. J. C 77(12), 906 (2017). https://doi.org/ 10.1140/epjc/s10052-017-5465-6

88. C. Shi, C. Mezrag, H.S. Zong, Pion and kaon valence quark distribution functions from Dyson-Schwinger equations. Phys. Rev. D 98(5), 054029 (2018). https://doi.org/10.1103/PhysRevD.98. 054029
89. C. Itzykson, J.B. Zuber, Quantum Field Theory. International Series In Pure and Applied Physics (McGraw-Hill, New York, 1980). https://doi.org/10.1063/1.2916419

90. D. Kapec, M. Perry, A.M. Raclariu, A. Strominger, Infrared divergences in QED. Revisited. Phys. Rev. D 96(8), 085002 (2017). https://doi.org/10.1103/PhysRevD.96.085002

91. R. Delbourgo, P.C. West, A gauge covariant approximation to quantum electrodynamics. J. Phys. A 10, 1049 (1977). https:// doi.org/10.1088/0305-4470/10/6/024

92. S. Jia, P. Maris, D.C. Duarte, T. Frederico, W. de Paula, E. Ydrefors, in 18th International Conference on Hadron Spectroscopy and Structure, pp. 560-564 (2020). https://doi.org/10. 1142/9789811219313_0095

93. V. Sauli, Gauge technique approximation to the $\pi \gamma$ production and the pion transition form factor. Phys. Rev. D 102(1), 014049 (2020). https://doi.org/10.1103/PhysRevD.102.014049

94. V. Sauli, Confinement within the use of Minkowski space integral representation (2020). arXiv:2011.00536

95. A. Kizilersu, T. Sizer, A.G. Williams, Regularization independent study of renormalized nonperturbative quenched QED. Phys. Rev. D 65, 085020 (2002). https://doi.org/10.1103/PhysRevD.65. 085020

96. C. Sturm, Y. Aoki, N.H. Christ, T. Izubuchi, C.T.C. Sachrajda, A. Soni, Renormalization of quark bilinear operators in a momentum-subtraction scheme with a nonexceptional subtraction point. Phys. Rev. D 80, 014501 (2009). https://doi.org/10. 1103/PhysRevD.80.014501

97. J.A. Gracey, Renormalization group functions of QCD in the minimal MOM scheme. J. Phys. A 46, 225403 (2013). https://doi.org/ $10.1088 / 1751-8113 / 46 / 22 / 225403$

98. J.S. Schwinger, Gauge invariance and mass. 2. Phys. Rev. 128, 2425 (1962). https://doi.org/10.1103/PhysRev.128.2425

99. C.J. Burden, J. Praschifka, C.D. Roberts, Photon polarization tensor in three-dimensional quantum electrodynamics. Phys. Rev. D 46, 2695 (1992). https://doi.org/10.1103/PhysRevD.46.2695

100. A. Aguilar, D. Binosi, J. Papavassiliou, The gluon mass generation mechanism: a concise primer. Front. Phys. (Beijing) 11(2), 111203 (2016). https://doi.org/10.1007/s11467-015-0517-6

101. J.S. Ball, T.W. Chiu, Analytic properties of the vertex function in gauge theories. 2.. Phys. Rev. D 22, 2550 (1980). https://doi.org/10.1103/physrevd.23.3085.2. https://doi. org/10.1103/PhysRevD.22.2550. [Erratum: Phys. Rev. D 23, 3085 (1981)]

102. N. Brown, N. Dorey, Multiplicative renormalizability and self consistent treatments of the Schwinger-Dyson equations. Mod. Phys. Lett. A 6, 317 (1991). https://doi.org/10.1142/ S0217732391000294

103. Z.H. Dong, H.J. Munczek, C.D. Roberts, Gauge covariant fermion propagator in quenched, chirally symmetric quantum electrodynamics. Phys. Lett. B 333, 536 (1994). https://doi.org/10.1016/ 0370-2693(94)90180-5

104. N. Brown, M.R. Pennington, Studies of confinement: how quarks and gluons propagate. Phys. Rev. D 38, 2266 (1988). https://doi. org/10.1103/PhysRevD.38.2266

105. N. Brown, M.R. Pennington, Studies of confinement: how the gluon propagates. Phys. Rev. D 39, 2723 (1989). https://doi.org/ 10.1103/PhysRevD.39.2723

106. D.S. Hwang, V.A. Karmanov, Many-body Fock sectors in WickCutkosky model. Nucl. Phys. B 696, 413 (2004). https://doi.org/ 10.1016/j.nuclphysb.2004.06.049

107. T. Frederico, G. Salmè, M. Viviani, Two-body scattering states in Minkowski space and the Nakanishi integral representation onto the null plane. Phys. Rev. D 85, 036009 (2012). https://doi.org/ 10.1103/PhysRevD.85.036009 
108. S. Sasagawa, H. Tanaka, Schwinger-Dyson equation in Minkowski space beyond the IE approximation. PTEP 2017(1), 013B04 (2017). https://doi.org/10.1093/ptep/ptw179

109. H. Tanaka, S. Sasagawa, Quark mass function in Minkowski space. PTEP 2017(12), 123B02 (2017). https://doi.org/10.1093/ $\mathrm{ptep} / \mathrm{ptx} 153$

110. D. Dudal, O. Oliveira, J. Rodriguez-Quintero, Nontrivial ghostgluon vertex and the match of RGZ, DSE and lattice Yang-Mills propagators. Phys. Rev. D 86, 105005 (2012). https://doi.org/10. 1103/PhysRevD.86.105005
111. F.T. Hawes, T. Sizer, A.G. Williams, On renormalized strong coupling quenched QED in four-dimensions. Phys. Rev. D 55, 3866 (1997). https://doi.org/10.1103/PhysRevD.55.3866

112. D. Binosi, R.A. Tripolt, Spectral functions of confined particles. Phys. Lett. B 801, 135171 (2020). https://doi.org/10.1016/j. physletb.2019.135171

113. E. Solis, C. Costa, V. Luiz, G. Krein, Quark propagator in Minkowski space. Few Body Syst. 60(3), 49 (2019). https://doi. org/10.1007/s00601-019-1517-9 